\begin{tabular}{|c|c|c|c|c|c|c|}
\hline \multirow{4}{*}{ Impact Factor: } & ISRA (India) & $=3.117$ & SIS (USA) & $=0.912$ & ICV (Poland) & $=6.630$ \\
\hline & ISI (Dubai, UAE & $=0.829$ & РИНЦ (Russia & $=0.156$ & PIF (India) & $=1.940$ \\
\hline & GIF (Australia) & $=0.564$ & ESJI (KZ) & $=8.716$ & IBI (India) & $=4.260$ \\
\hline & JIF & $=1.500$ & SJIF (Morocco & $=5.667$ & OAJI (USA) & $=0.350$ \\
\hline
\end{tabular}

\section{SOI: $1.1 /$ TAS DOI: $10.15863 /$ TAS \\ International Scientific Journal Theoretical \& Applied Science}

p-ISSN: 2308-4944 (print) e-ISSN: 2409-0085 (online)

Year: 2019 Issue: 03 Volume: 71

Published: $25.03 .2019 \quad$ http://T-Science.org

SECTION 2. Applied mathematics.

Mathematical modeling.
QR - Issue

QR - Article

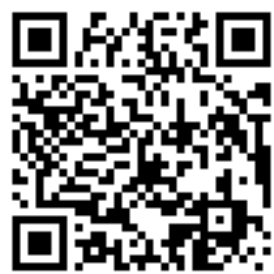

Corresponding Member of Internation

Theoretical and Applied Sciences (USA), Candidate of physics and mathematical sciences,

Department «Information

technologies and automation», Professor,

Noncommercial joint-stock company

"Kazakh national agrarian university", Kazakhstan

\title{
A MATRIX OF VALUES THE COEFFICIENTS OF COMBINATIONAL PROPORTIONALITY
}

\begin{abstract}
The article solved a new spectral problem - ISP 6, inverse to the Direct Spectral Problem [3]. For known values of the eigenvalues $\lambda_{1}, \ldots, \lambda_{n}, \Lambda_{n n}=\operatorname{diag}\left(\lambda_{1}, \ldots, \lambda_{n}\right)$ such that $\lambda_{1}>\ldots>\lambda_{n}>0$ and the known and given values of some components of the $n$ eigenvectors $c_{1}, \ldots, c_{n}$ from the matrix $C_{n n}=\left[c_{1}|\ldots| c_{n}\right]$ of eigenvectors to find:1) new model values of known, but not given, values of the components of $n$ pseudoeigen vectors $c^{+}{ }_{\ell+1}, \ldots, c^{+}{ }_{n,}, c^{+}{ }_{j}=\left(c^{+}{ }_{1 j}, c^{+}{ }_{2 j} \ldots c^{+}{ }_{n j}\right)^{T}, j=1, \ldots, n$, from the new matrix ${ }^{+}{ }_{n n}=\left[c^{+} 1|\ldots| c^{+}{ }_{n}\right]$ pseudoeigen vectors;

2) the resulting matrix of eigenvalues $\Lambda{ }^{+}{ }_{n n}$ should have the values equal to $1: \Lambda{ }^{+}{ }_{n n}=\operatorname{diag}(1, \ldots,, 1)=I_{n n}$.

$3)$ the resulting full matrix of eigenvalues $\Lambda^{+}{ }_{n n}=I_{n n}$ and the matrix of pseudoeigen vectors $C^{+}{ }_{n n}=\left[c^{+}{ }^{+}|\ldots| c^{+}{ }_{n}\right]$ must satisfy the equalities $C^{+T}{ }_{n n} C^{+}{ }_{n n} \neq I_{n n}, C^{+}{ }_{n n} C^{+T}{ }_{n n}=I_{n n}, \quad C^{+}{ }_{n n} \Lambda^{+}{ }_{n n} C^{+T}{ }_{n n}=I_{n n}, \quad c_{j}^{+} \Lambda^{+}{ }_{n n} c_{j}^{+T}=1, c_{i}^{+} \Lambda^{+}{ }_{66} c_{j}^{+T}=r^{+}{ }_{i j}=0, r^{+}{ }_{i j}=r^{+}{ }_{j i}=0$, $i=1, \ldots, n ; j=1, \ldots, n, i \neq j$, and the correlation matrix $R^{+}{ }_{n n}=C^{+}{ }_{n n} \Lambda^{+}{ }_{66} C^{+T_{n n}}=I_{n n}$ should have new matrices of pseudoeigen vectors ${ }^{C+}{ }_{n n} \neq I_{n n}$ and eigenvalues $\Lambda^{+}{ }_{n n}=I_{n n}=\operatorname{diag}(1, \ldots, 1), \lambda^{+}{ }_{1}=\ldots=\lambda^{+}{ }_{n}=1, \lambda_{1}+\ldots+\lambda^{+}{ }_{n}=n$. In ISP 6 introduced new terms "coefficient of combinational proportionality" (CCP), " pseudoeigen vectors". In the developed models ISP 4, ISP ISP5, ISP 6, Optimization Problems were formulated and solved with the corresponding assumptions on their parameters and variables. Model $C^{+}{ }_{n n}$-samples $Z^{(t)}{ }_{m n}=U^{(t)}{ }_{m n}\left[C^{+}{ }_{n n}\right]^{T}, t=1, \ldots, k_{t}$, (reproduced not by the known spectrum of the correlation matrix) were used to solve the problems of "extracting digital knowledge "of digital data from different subject areas, means of cognitive modeling. An example of modeling and visualization of a "figure from $m$ points" inside an $n$ dimensional bal is given. It illustrates model uncorrelated z-variables corresponding to their cognitively independent meanings.

Key words: coefficient of combinational proportionality, pseudoeigen vectors, $C^{+}$-sample, $\Lambda$-sample.

Language: Russian

Citation: Zhanatauov, S. U. (2019). A matrix of values the coefficients of combinational proportionality. ISJ Theoretical \& Applied Science, 03 (71), 401-419.

Soi: http://s-o-i.org/1.1/TAS-03-71-31 Doi: crossef https://dx.doi.org/10.15863/TAS.2019.03.71.31

\section{МАТРИЦА ЗНАЧЕНИЙ КОЭФФИЦИЕНТОВ КОМБИНАЦИОННОЙ ПРОПОРЦИОНАЛЬНОСТИ}

Аннотация: В статье решена новая спектральная задача - ОСЗ 6, обратная к Прямой Спектральной Задаче [3]. Для известных значений собственных чисел $\lambda_{1}, \ldots, \lambda_{n}, \Lambda_{n n}=\operatorname{diag}\left(\lambda_{1}, \ldots, \lambda_{n}\right)$ maких, что $\lambda_{1}>\ldots>\lambda_{n}>0 u$ известных и заданных значений некоторых компонент $y$ n собственных векторов $c_{1}, \ldots, c_{n}$ из матрицы $C_{n n}=\left[c_{1}|\ldots| \boldsymbol{c}_{n}\right]$ собственных векторов найти: 1)новые модельные значения известным, но не заданным значениям

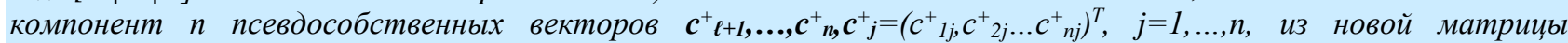
$C^{+}{ }_{n n}=\left[c^{+} 1|\ldots| c_{n}^{+}\right]$псевдособственных векторов;2) полученная матрица собственных чисел $\Lambda^{+}{ }_{n n}=\operatorname{diag}(1, \ldots,, 1)$ должна иметь значения, равные $\left.1: \Lambda^{+}{ }_{n n}=\operatorname{diag}(1, \ldots, 1)=I_{n n} ; 3\right)$ полученные полные матрица собственных чисел $\Lambda_{n n}^{+}=I_{n n} \quad$ и матрица псевдособственных векторов $C^{+}{ }_{n n}=\left[c^{+} 1|\ldots| c^{+}{ }_{n}\right]$ должны удовлетворять равенствам
\end{abstract}




\begin{tabular}{|c|c|c|c|c|c|c|}
\hline \multirow{4}{*}{1101} & ISRA (India) & $=3.117$ & SIS (USA) & $=0.912$ & ICV (Poland) & $=6.630$ \\
\hline & ISI (Dubai, UAE & $=0.829$ & РИНЦ (Russia) & $=0.156$ & PIF (India) & $=1.940$ \\
\hline & GIF (Australia) & $=0.564$ & ESJI (KZ) & $=8.716$ & IBI (India) & $=4.260$ \\
\hline & JIF & & & & OAJI (USA) & $=0.350$ \\
\hline
\end{tabular}

$C^{+T}{ }_{n n} C^{+}{ }_{n n} \neq I_{n n}, C^{+}{ }_{n n} C^{+T}{ }_{n n}=I_{n n}, \quad C^{+}{ }_{n n} \Lambda^{+}{ }_{n n} C^{+T_{n n}}=I_{n n}, \quad c_{j}^{+} \Lambda^{+}{ }_{n n} c_{j}^{+T}=1, \quad c_{i}^{+} \Lambda^{+} n n c^{+T}=r^{+}{ }_{i j}=0, r^{+}{ }_{i j}=r^{+}{ }_{j i}=0, i=1, \ldots, n ; j=1, \ldots, n, i \neq j, \quad a$ корреляционная матрица $R^{+}{ }_{n n}=C^{+}{ }_{n n} \Lambda^{+}{ }_{66} C^{+T}{ }_{n n}=I_{n n}$ должна иметь новые матрищы псевдособственных векторов $C^{+}{ }_{n n} \neq I_{n n}$ и собственных чисел $\Lambda^{+}{ }_{n n}=I_{n n}=\operatorname{diag}(1, \ldots, 1), \lambda^{+}{ }_{l}=\ldots=\lambda^{+}{ }_{n}=1, \lambda_{1}+\ldots+\lambda^{+}{ }_{n}=n . B$ модели ОСЗб введены новые термины «коэффиииент комбинаиионной пропорииональности» (ККП), «псевдосоственные векторы». В разработанных моделях ОСВ4,ОС 35 , ОСЗ6 сформулированы и решены Оптимизационные Задачи $c$

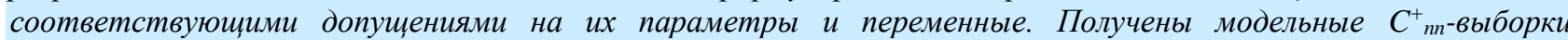
$Z^{(t)}{ }_{m n}=U^{(t)}{ }_{m n}\left[C^{+}{ }_{n n}\right]^{T}, t=1, \ldots, k_{t}$, (воспроизведенные не по известному спектру корреляционной матрицы), используемые при решении задач «извлечения цифровых знаний» из иифровых данных из разных предметных областей, средствами когнитивного моделирования. Приведен пример моделирования и визуализации «фигуры из m точек» внутри п-мерного шара. Он иллюстрирует модельные некоррелированные z-переменные, соответствующие их когнитивные независимым смыслам .

Ключевые слова: коэффициент комбинационной пропорииональности, псевдособственные векторы, С+выборка, $\Lambda$-выборка.

\section{Введение}

Часто в текстах публикаций по анализу реальных данных методом главных компонент (ПМ ГК [1,2]) не приводятся неиспользуемые для содержательной интерпретации компоненты собственных векторов из матрицы $\mathbf{C}_{n n}=\left[\mathbf{c}_{1}\left|\mathbf{c}_{2}\right| \ldots \mid \mathbf{c}_{\mathbf{n}}\right]$. Значения компонент собственных векторов выборочной корреляционной матрицы $\mathrm{R}_{\mathrm{nn}}$ анализируемой реальной многомерной стандартизованной выборки $Z_{\mathrm{mn}}$ зависят от от элементов $\lambda_{1}, \ldots, \lambda_{\mathrm{n}}$ спектра $\Lambda_{\mathrm{nn}}=\operatorname{diag}\left(\lambda_{1}, \lambda_{2}, \ldots, \lambda_{\ell}, \ldots, \lambda_{\mathrm{n}}\right)$ (примеры спектров [2,стр.177-183]). Пары матриц $\left(\mathrm{C}_{\mathrm{nn}}, \Lambda_{\mathrm{nn}}\right)$ согласованы между собой посредством третьей матрицы $R_{n n}$ через равенство $R_{n n} C_{n n}=C_{n n} \Lambda_{n n}$. Если известна матрица $\mathrm{R}_{\mathrm{nn}}$, то решаем прямую спектральную задачу ПСЗ [2], а если известен спектр $\Lambda_{\mathrm{nn}}$, то решаем ОС31 [3]: $\Lambda_{\mathrm{nn}}=>\left(\mathrm{C}^{(\ell)}{ }_{\mathrm{nn}}, \mathrm{R}^{(\ell)}{ }_{\mathrm{nn}}\right)$, $\ell=1, \ldots, \mathrm{k}_{\ell}<\infty$, для единственного решения $\Lambda_{\mathrm{nn}}$ из ПС3. Спектр $\Lambda_{\mathrm{nn}}=\operatorname{diag}\left(\lambda_{1}, \lambda_{2}, \ldots, \lambda_{\mathrm{n}}\right) \quad$ является решением прямой спектральной задачи (ПСЗ) диагонализации симметрической матрицы $\mathrm{R}_{\mathrm{nn}}=>\left(\mathrm{C}_{\mathrm{nn}}, \Lambda_{\mathrm{nn}}\right)$. В ПСЗ (однородной спектральной задаче), решаемой для симметрической матрицы $\mathrm{R}_{\mathrm{nn}}=\mathrm{R}^{\mathrm{T}}{ }_{\mathrm{nn}}$ вычисляются 2 матрицы: ортогонормированная матрица $\mathrm{C}_{\mathrm{nn}}$ собственных векторов $\mathbf{c}_{\mathbf{j}}=\left(\mathrm{c}_{1 \mathrm{j}}, \mathrm{c}_{2 \mathrm{j}} \ldots \mathrm{c}_{\mathrm{nj}}\right)^{\mathrm{T}}$, расположенных по её столбцам: $\quad \mathbf{C}_{\mathrm{nn}}=\left[\mathbf{c}_{1}\left|\mathbf{c}_{2}\right| \ldots \mid \mathbf{c}_{\mathbf{n}}\right] \quad$ и матрица $\Lambda_{\mathrm{nn}}$ собственных чисел матрицы $\mathrm{R}_{\mathrm{nn}}$. Эти матрицы $\left(\mathrm{C}_{\mathrm{nn}}\right.$ и $\left.\mathrm{R}_{\mathrm{nn}}\right)$ согласованы со спектром $\Lambda_{\mathrm{nn}}=\operatorname{diag}\left(\lambda_{1}, \ldots, \lambda_{\mathrm{n}}\right)$ корреляционной матрицы $\mathrm{R}_{\mathrm{nn}}$ таким образом, что выполняются равенства $\mathrm{R}_{\mathrm{nn}} \mathrm{C}_{\mathrm{nn}}=\mathrm{C}_{\mathrm{nn}} \Lambda_{\mathrm{nn}}$, $\mathrm{C}^{\mathrm{T}}{ }_{\mathrm{nn}} \mathrm{C}_{\mathrm{nn}}=\mathrm{C}_{\mathrm{nn}} \mathrm{C}_{\mathrm{nn}}^{\mathrm{T}}=\mathrm{I}_{\mathrm{nn}}, \quad \operatorname{diag}\left(\mathrm{R}_{\mathrm{nn}}\right)=(1, \ldots, 1)$, $\operatorname{tr}\left(\mathrm{R}_{\mathrm{nn}}\right)=1+1+\ldots+1=\operatorname{tr}\left(\Lambda_{\mathrm{nn}}\right)=\lambda_{1}+\ldots+\lambda_{\mathrm{n}}=\mathrm{n}, \lambda_{1} \geq \ldots \geq \lambda_{\mathrm{n}} \geq 0$. Для моделирования входного объекта ОСЗ1 спектра $\Lambda_{\mathrm{nn}}$, в работе [4] были разработана модель ОС3, где моделировались спектры с заданными значениями его $\mathrm{f}$-параметров. Без учета влияющей на $\left(\mathrm{f}_{1}, \mathrm{f}_{2}, \mathrm{f}_{3}, \mathrm{f}_{4}, \mathrm{f}_{5}, \mathrm{f}_{6}\right)$-спектр матрицы $\mathbf{C}_{\mathrm{nn}}$ собственных векторов. В статьях [5-7] моделируются спектры с другими значениями $\mathrm{f}$-параметров: $\left(\mathrm{f}_{1}, \mathrm{f}_{2}, \mathrm{f}_{4}\right)$-спектр [5], восстанавливающий полный спектр таким, что значения его $\mathrm{f}$-параметров $\mathrm{f}_{1}\left(\mathrm{R}_{\mathrm{nn}}\right), \mathrm{f}_{2}\left(\mathrm{R}_{\mathrm{nn}}\right), \mathrm{f}_{4}\left(\mathrm{R}_{\mathrm{nn}}\right)$ имеют требуемые значения $\mathrm{f}_{1}, \mathrm{f}_{2}, \mathrm{f}_{4}$. Эти и другие модели [1] моделируют матрицу $\Lambda_{\mathrm{nn}}$ собственных чисел без учета ее связи с матрицей $\mathrm{C}_{\mathrm{nn}}$ собственных векторов.

В этой статье продолжим исследование ОС3, использующих связи матриц $\Lambda_{\mathrm{nn}}$ и $\mathbf{C}_{\mathrm{nn}}$ между собой, $\mathrm{T}$ е будем рассматривать пару матриц $\left(\mathrm{C}_{\mathrm{nn}}, \Lambda_{\mathrm{nn}}\right)$. Целью получения модельных решений задач моделирования спектра является получение модельной $\Lambda$-выборки $Z^{(\ell, t)}{ }_{m n}$, целью получения решения $\mathrm{C}^{+}{ }_{n n}-$ матрицы псевдособственных векторов, как показано ниже, является получение модельной $\mathrm{C}^{+}{ }_{\mathrm{nn}}$-выборки. Наши модельные $\mathrm{C}^{+}{ }_{\mathrm{nn}}^{-}$ выборки $\mathrm{Z}^{(\mathrm{t})}{ }_{\mathrm{mn}}=\mathrm{U}^{(\mathrm{t})}{ }_{\mathrm{mn}}\left[\mathrm{C}^{+}{ }_{\mathrm{nn}}\right]^{\mathrm{T}}$, воспроизведенные не по известному спектру корреляционной матрицы, применяются при решении задач «извлечения цифровых знаний» из цифровых данных. Начальными входными объектами являются 2 матрицы, образующие взаимосвязанную пару матриц $\left(\mathrm{C}_{\mathrm{nn}}, \Lambda_{\mathrm{nn}}\right)$. Дополним ряд моделей OC3 из статьи тремя моделями : ОС 34, ОС 35, ОС36. Эти другие задачи моделируют матрицы собственных векторов $\mathrm{C}_{\mathrm{nn}}$, а в модели ОС36 решена задача моделирования матрицы псевдособственных векторов $\mathrm{C}^{+}$n.

При решении оптимизационных задач из ОС3 вида $(\ldots)=>\Lambda_{\text {nn }}$ в качестве переменных и постоянных используются только элементы спектра. Как выяснилось при решении других задач [6-10] необходимо использовать и матрицу собственных векторов $\mathrm{C}_{\mathrm{nn}}$. В статье [10] впервые используется матрица собственных векторов $\mathrm{C}_{\mathrm{nn}}$. Для когнитивного алгоритма назначения имен (смыслов) показателей индивидуального сознания использована пара матриц $\left(\Lambda_{\mathrm{nn}}, \mathrm{C}_{\mathrm{nn}}\right)$. Разработанные модели ОС3 расширяют перечень обратных задач [11] и используются при решении задач «извлечения цифровых знаний» из цифровых данных из разных предметных областей средствами когнитивного моделирования.

\section{Постановка задачи}

Рассмотрим случай $\Lambda_{\mathrm{nn}}=\mathrm{I}_{\mathrm{nn}}, \lambda_{1}=\ldots=\lambda_{\mathrm{n}}=1$. Равенство собственных чисел другу означает 


\begin{tabular}{|c|c|c|c|c|c|c|}
\hline \multirow{4}{*}{ Impact Factor: } & ISRA (India) & $=3.117$ & SIS (USA) & $=0.912$ & ICV (Poland) & $=6.630$ \\
\hline & ISI (Dubai, UAF & $=0.829$ & РИНЦ (Russia & $=0.156$ & PIF (India) & $=1.940$ \\
\hline & GIF (Australia) & $=0.564$ & ESJI (KZ) & $=8.716$ & IBI (India) & $=4.260$ \\
\hline & JIF & $=1.500$ & SJIF (Morocce & $=5.667$ & OAJI (USA) & $=0.350$ \\
\hline
\end{tabular}

равенство дисперсий $\mathrm{y}$-переменных одной константе, означающее равенство количества информации, содержащейся в каждом из них, друг другу. Такая гипотеза выражается через элементы спектра $\Lambda_{\mathrm{nn}}=\mathrm{I}_{\mathrm{nn}}$. Возникает вопрос: как это свойство элементов спектра повлияет на элементы матрицы собственных векторов $\mathrm{C}_{\mathrm{nn}}$ ? Существование системы взаимно перпендикулярных единичной длины векторов, исходящих из центра n-мерного шара, можно мысленно представить. Но таких систем векторов существует в бесконечном количестве. Как их можно смоделировать? Зачем нам нужны такие по-разному ориентированные взаимно перпендикулярные единичной длины векторы, исходящие из центра n-мерного шара? Если эта система векторов находится в центре гиперэллипсоида, то существует только одна система. Она отыскивается вычислительным способом путем решения ПСЗ: $\mathrm{R}_{\mathrm{nn}}=>\left(\mathrm{C}_{\mathrm{nn}}, \Lambda_{\mathrm{nn}}\right)$. Вместо системы Если известна симметрическая матрица $\mathrm{R}_{\mathrm{nn}}=(1 / \mathrm{m}) \mathrm{Z}_{\mathrm{mn}}^{\mathrm{T}} \mathrm{Z}_{\mathrm{mn}}$ и стандартизованая выборка $Z_{\mathrm{mn}}$ значений $\mathrm{Z}$ переменных, то реализуема ПСЗ. Но мы рассматриваем модели Обратных Спектральных Задач, когда не известны матрица $\mathrm{R}_{\mathrm{nn}}$ и $\mathrm{C}_{\mathrm{nn}}$.

Используя вышеприведенные суждения в модели ПСЗ вида $\mathrm{R}^{+}{ }_{n n} \mathrm{C}^{+}{ }_{n n}=\mathrm{C}^{+}{ }_{n n} \Lambda^{+}{ }_{n n}$ полагаем $\mathrm{R}^{+}{ }_{n n}=\mathrm{I}_{\mathrm{nn}}$ $\Lambda^{+}{ }_{n n}=\mathrm{I}_{\mathrm{nn}}$ и имеем равенство $\mathrm{nn} \quad \mathrm{I}_{\mathrm{nn}} \mathrm{C}^{+}{ }_{\mathrm{nn}}=\mathrm{C}^{+}{ }_{\mathrm{nn}} \mathrm{I}_{\mathrm{nn}}$. Данное равенство можно изобразить в виде $\mathrm{C}^{+}{ }_{n n}=$ $\mathrm{C}^{+}{ }_{n n}$, что демонстрирует отсутствие входного объекта в нашей модели ОС3, но наличие выходного объекта $\mathrm{C}^{+}{ }_{n n}$. На неизвестное решение $\mathrm{C}^{+}{ }_{n n}$ введем ограничения вида $\mathrm{C}^{+}{ }_{n n} \mathrm{C}^{+\mathrm{T}}{ }_{n n}=\mathrm{I}_{\mathrm{nn}}$. Так как в матрице $\mathrm{C}^{+}{ }_{n n}$ собственные векторы $\mathbf{c}^{+}{ }_{\mathrm{j}}=\left(\mathrm{c}^{+}{ }_{1 \mathrm{j}}, \mathrm{c}^{+}{ }_{2 \mathrm{j}} \ldots \mathrm{c}^{+}{ }_{\mathrm{nj}}\right)^{\mathrm{T}}$, взаимно ортогональны $\mathbf{c}^{+}{ }_{j} \mathbf{c}^{+}{ }_{j}=0$, при $\mathrm{i} \neq \mathrm{j}$, но не имеют единичную дину $\mathbf{c}_{j}^{+\mathrm{T}} \mathbf{c}^{+} \neq 1$. Это условие $\mathbf{c}_{\mathrm{j}}^{\mathrm{T}} \mathbf{c}_{\mathrm{j}}$ $\neq 1$ формализует свойства системы взаимно перпендикулярных неединичной длины векторов. Мы требуем построения системы взаимно перпендикулярных векторов разной длины, исходящих из центра n-мерного шара. Условие $\mathbf{c}_{\mathbf{j}}^{+\mathrm{T}} \mathbf{c}^{+} \neq 1, \mathbf{j} \in\{1, \ldots, \mathrm{n}\}$, означает наличие векторов $\mathbf{c}^{+}{ }_{j}=\left(\mathrm{c}^{+}{ }_{1 \mathrm{j}}, \mathrm{c}^{+}{ }_{2 \mathrm{j}} \ldots \mathrm{c}^{+}{ }_{\mathrm{nj}}\right)^{\mathrm{T}}$ разной длины. Фиксировать значения их длин пока не будем. В модельной матрице $\mathrm{C}^{+}{ }_{n n}$ не будем требовать единичной дины для всех векторов $\mathbf{c}_{\mathbf{j}}{ }^{+}=\left(\mathrm{c}^{+}{ }_{1 \mathrm{j}}, \mathrm{c}^{+}{ }_{2 \mathrm{j}} \ldots \mathrm{c}^{+}{ }_{\mathrm{nj}}\right)^{\mathrm{T}} \mathbf{c}_{\mathrm{j}}{ }^{+\mathrm{T}} \mathbf{c}_{\mathrm{j}}{ }^{+} \neq 1$, $\mathrm{j} \in\{1, \ldots, n\}$. Это более слабое ограничение на переменных (изменяемых) модели ОС36 реализовано ниже, оно реализовано в программетаблице процедуры Solver, Собственные векторы $\mathbf{c}^{+}{ }_{\mathbf{j}}=\left(\mathrm{c}^{+}{ }_{1 \mathrm{j}}, \mathrm{c}^{+}{ }_{2 \mathrm{j}} \ldots \mathrm{c}^{+}{ }_{\mathrm{nj}}\right)^{\mathrm{T}}$, назовем псевдособственным вектором, а матрицу $\mathrm{C}^{+}{ }_{n n}$ с новыми свойствами назовем матрицей псевдособственных векторов.

\footnotetext{
Многомерные выборки, содержащие заданные индикаторы наличия смыслов переменных
}

При добыче знаний из числовых данных используются индикаторы наличия смысла у zпеременной. Индикаторы наличия смыслов zпеременных находятся среди $\mathrm{n}$ слагаемых $\mathrm{z}_{\mathrm{i} 1} \mathrm{c}_{1 \mathrm{j}}$, $\mathrm{Z}_{\mathrm{i} 2} \mathrm{c}_{2 \mathrm{j}}, \ldots, \mathrm{Z}_{\mathrm{in}} \mathrm{c}_{\mathrm{nj}}$. Иx сумма образует $\mathrm{y}$-переменную $\mathrm{y}_{\mathrm{ij}}=\mathrm{z}_{\mathrm{i} 1} \mathrm{c}_{1 \mathrm{j}}+\mathrm{z}_{\mathrm{i} 2} \quad \mathrm{c}_{2 \mathrm{j}}+\ldots+\mathrm{z}_{\mathrm{in}} \mathrm{c}_{\mathrm{nj}}, \quad$ имеющую свой «суммарный» смысл. Индикатором наличия смысла у k-ой z-переменной $\mathrm{z}_{\mathrm{k}}$ является весомость значения $\mathrm{abs}\left(\mathrm{c}_{\mathrm{kj}}\right)$ при $\mathrm{k}$-ой $\mathrm{z}$-переменной: $\operatorname{abs}\left(c_{2 j}\right) \geq c(j)$. Этот индикатор ищется в произведении вида $\mathrm{z}_{\mathrm{i} 2} \mathrm{c}_{2 \mathrm{j}}$. Здесь постоянная величина $\mathrm{c}(\mathrm{j})>0$ является пороговой константой для всех компонент j-го собственного вектора, присутствующих в значении $у$-переменной $\mathrm{y}_{\mathrm{ij}}=\mathrm{z}_{\mathrm{i} 1} \mathrm{c}_{1 \mathrm{j}}+\mathrm{z}_{\mathrm{i} 2} \quad \mathrm{c}_{2 \mathrm{j}}+\ldots+\mathrm{z}_{\mathrm{in}} \mathrm{c}_{\mathrm{nj}}$. Индикатор $\operatorname{abs}\left(\mathrm{c}_{\mathrm{kj}}\right) \geq \mathrm{c}(\mathrm{j})$ при $\mathrm{k}$-ой $\mathrm{z}$-переменной показывает наличие смысла, имя $\mathrm{k}-\mathrm{oй} \mathrm{z}$-переменной отражает ее смысл. Значение $\mathrm{z}_{\mathrm{ik}} \mathrm{k}$-ой $\mathrm{z}$-переменной измеряет изменчивость «веса» $\mathrm{c}_{\mathrm{kj}}$. Выделяют только слагаемые с значимыми «весами» $\mathrm{c}_{1 \mathrm{j}}, \mathrm{c}_{2 \mathrm{j}}, \ldots, \mathrm{c}_{\mathrm{nj}}[1-4$ ], соответствующих $\ell$ доминирующим собственным

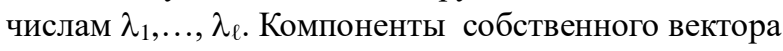
равны значениям (y,z)-коэффициентов корреляции. Если j-номер собственного вектора, то для процесса извлечения знаний целесообразно переписать собственный вектор $\mathbf{c}_{\mathbf{j}}=\left(\mathrm{c}_{1 \mathrm{j}}, \ldots, \mathrm{c}_{\mathrm{nj}}\right)^{\mathrm{T}}$ в другом понятном для когнитивного восприятия виде- $\boldsymbol{c}_{\mathrm{j}}=\left(\operatorname{corr}\left(\mathrm{y}_{\mathrm{j}}, \mathrm{z}_{1}\right), \quad \operatorname{corr}\left(\mathrm{y}_{\mathrm{j}}, \mathrm{z}_{2}\right), \ldots, \quad \operatorname{corr}\left(\mathrm{y}_{\mathrm{j}}, \mathrm{z}_{\mathrm{n}}\right)\right) \quad{ }^{\mathrm{T}}$. Числовые данные при добыче знаний должны образовывать систему из из матрицы $\mathrm{C}^{+}{ }_{\mathrm{nn}}$ и матрицы значений изменчивостей $\mathrm{Z}_{\mathrm{mn}}$. Такая терминология будет употребляться в когнитивном моделировании. Ранее матрица $Z_{m n}$ значений стандартизованных $\mathrm{z}-$ переменных, соответствующих матрице числовых данных $\mathrm{X}^{0}{ }_{\mathrm{mn}}$.

Наличие только матрицы псевдособственных векторов $\mathrm{C}^{+}{ }_{\mathrm{nn}} \quad$ с свойством $\mathrm{C}^{+}{ }_{n n} \mathrm{C}^{+\mathrm{T}}{ }_{\mathrm{nn}}=\mathrm{I}_{\mathrm{nn}}$., ортогональными псевдособственными векторами: $\mathbf{c}_{\mathrm{j}}^{+} \mathbf{c}^{+}{ }_{\mathrm{j}}=0$, при $\mathrm{i} \neq \mathrm{j}$, позволяет моделировать, n-мерные выбоки $\mathrm{Y}_{\mathrm{mn}} \mathrm{y}$-переменных, $\mathrm{n}$-мерные выборки $\mathrm{Z}_{\mathrm{mn}} \mathrm{z}$-переменных. Используя матрицу $\mathrm{C}^{+}{ }_{\mathrm{nn}}$ из модели ОС36 мы специально моделируем матрицу $\mathrm{Z}_{\mathrm{mn}} \mathrm{Z}$-переменных, специально моделируем матрицу $\mathrm{Y}_{\mathrm{mn}}$ у-переменных, имеющих корреляционные матрицы, равные $\mathrm{I}_{\mathrm{nn}}=\operatorname{diag}(1, \ldots, 1):(1 / \mathrm{m}) \mathrm{Y}^{\mathrm{T}}{ }_{\mathrm{mn}} \mathrm{Y}_{\mathrm{mn}}=\mathrm{I}_{\mathrm{nn}}$, $(1 / \mathrm{m}) Z^{\mathrm{T}}{ }_{\mathrm{mn}} Z_{\mathrm{mn}}$, где используем декоррелированную выборку $\mathrm{U}_{\mathrm{nn}}$ из ОМ ГК [1-3] такую, что $(1 / \mathrm{m}) \mathrm{U}^{\mathrm{T}}{ }_{\mathrm{mn}} \mathrm{U}_{\mathrm{mn}}=\mathrm{I}_{\mathrm{nn}} . \quad \mathrm{Y}_{\mathrm{mn}}=\mathrm{U}_{\mathrm{nn}}, \mathrm{Z}_{\mathrm{mn}}=\mathrm{Y}_{\mathrm{mn}} \mathrm{C}^{+\mathrm{T}}{ }_{\mathrm{nn}}=\mathrm{U}_{\mathrm{nn}} \mathrm{C}^{+\mathrm{T}}{ }_{\mathrm{nn}}$, где специально решаем Оптимизационную Задачу. Решению ОСЗ6 предшествовали решения других OC3: ОС34, ОС35.

Эволюция представлений (взглядов) на матрицу $\mathrm{C}_{\mathrm{nn}}$, собственных векторов и на матрицу $\mathrm{C}^{+}{ }_{n n}$, псевдособственных векторов менялась в процессах разработки, применений моделей ОС 32 , ОС 33, ОС34, ОС 35,ОС36. И в модели ОС36 значения элементов $\quad c_{\mathrm{kj}}=\operatorname{corr}\left(\mathrm{z}_{\mathrm{k}}, \mathrm{y}_{\mathrm{j}}\right) \quad$ матрицы $\quad \mathrm{C}^{+}{ }_{\mathrm{nn}}$ псевдособственных векторов интерпретируются 


\begin{tabular}{|c|c|c|c|c|c|c|}
\hline \multirow{4}{*}{ Impact Factor: } & ISRA (India) & $=3.117$ & SIS (USA) & $=0.912$ & ICV (Poland) & $=6.630$ \\
\hline & ISI (Dubai, UAI & $=0.829$ & РИНЦ (Russia) & $=0.156$ & PIF (India) & $=1.940$ \\
\hline & GIF (Australia) & $=0.564$ & ESJI (KZ) & $=8.716$ & IBI (India) & $=4.260$ \\
\hline & JIF & $=1.500$ & SJIF (Morocco) & $=5.667$ & OAJI (USA) & $=0.350$ \\
\hline
\end{tabular}

как значения $\mathrm{c}_{\mathrm{kj}}, \mathrm{k}=1, \ldots, \mathrm{n}, \quad$ коэффициентов КОМБИНАЦИОННОЙ

ПРОПОРЦИОНАЛЬНОСТИ (весомых «весов») при значениях $\mathrm{Z}_{\mathrm{ik}}, \quad \mathrm{k}=1, \ldots, \mathrm{n}, \quad$ изменчивостей $\mathrm{z}-$ переменных. Значения $\mathrm{z}_{\mathrm{ik}}, \mathrm{i}=1, \ldots, \mathrm{m}, \mathrm{k}=1, \ldots, \mathrm{n}, \mathrm{z}-$ переменных из $\mathrm{n}$-мерной выборки $\mathrm{Z}_{\mathrm{mn}}$ интерпретируются (рассматриваются при когнитивном моделировании) как значения пропорциональных изменчивостей пар переменных $\mathrm{r}_{\mathrm{ij}}=\operatorname{corr}\left(\mathrm{z}_{\mathrm{i}}, \mathrm{Z}_{\mathrm{j}}\right): \quad \mathrm{z}_{\mathrm{ki}}=\mathrm{r}_{\mathrm{ij}} \mathrm{z}_{\mathrm{kj}}$. Матрица $\mathrm{C}^{+}{ }_{\mathrm{nn}}$ значений весомых «весов» и пренебрежимо малых «весов» является основным источником цифровых знаний [6,12-15]. В этих исследованиях по когнитивному моделированию и извлечению цифровых знаний анализу подвергается матрица $\mathrm{C}_{\mathrm{nn}}$. Применены [6,12-15] разные критерии для разных доминирующих значений собственных чисел выделены значения весомых «весов». Основными объектами анализа является пара матриц $\left(\Lambda_{\mathrm{nn}}, \mathrm{C}_{\mathrm{nn}}\right)$, причем у матрицы $\Lambda_{\mathrm{nn}}$ учитываются только первые $\ell$ доминирующих элемента. Замечено [6,12-15], что для реальных данных верна гипотеза: количество выделяемых (по критерию) компонент j-го собственного вектора пропорционально величине $\lambda_{\mathrm{j}}$ собственного числа. Так как реальность многообразна, а мы анализировали сравнительно малое количество реальных таблиц объект-свойства, то не можем подтвердить истинность гипотезы. Принято предполагать: чем больше величина $\lambda_{\mathrm{j}}$, тем большее количество выделенных (по критерию) компонент j-го собственного вектора. Известно, что чем больше значение дисперсии $\lambda_{\mathrm{j}}$ j-ой $\mathrm{y}$ переменной, тем большее количество значений $\mathrm{y}-$ переменной превышает пороговое значение у : |у ${ }_{\mathrm{ki}} \mid \geq \mathrm{y}_{0}, \mathrm{k} \in\{1, \ldots, \mathrm{m}\}$.

Пока будем руководствоваться гипотезой: количество выделяемых (по критерию) компонент j-го собственного вектора, превышающего пороговое значение $\mathrm{c}_{0}:\left|\mathrm{c}_{\mathrm{kj}}\right| \geq \mathrm{c}_{0}, \quad \mathrm{k} \in\{1, \ldots, \mathrm{n}\}$, пропорционально величине $\lambda_{j}$ j-го собственного числа. При решении обратной задачи моделирования матрицы $\mathrm{C}_{\mathrm{nn}}$ собственных векторов необходимо следовать вышеприведенной гипотезе. $\mathrm{B}$ модельной матрице $\mathrm{C}_{\mathrm{nn}}$ собственных векторов ее элементы должны удовлетворять условиям гипотезы.

Ниже в модели ОС 36 построена такая матрицаэто матрица $\mathrm{C}^{+}{ }_{\mathrm{nn}}$ псевдособственных векторов. Модель ОСЗ6 позволит моделировать матрицу $\mathrm{Z}_{\mathrm{mn}}$ значений $\mathrm{z}$-переменных, интерпретируемых (при когнитивном моделировании) как значений коэффициентов пропорциональной изменчивости в паре переменных $\quad \mathrm{r}_{\mathrm{ij}}=\operatorname{corr}\left(\mathrm{z}_{\mathrm{i}}, \mathrm{z}_{\mathrm{j}}\right)$ : $\quad \mathrm{z}_{\mathrm{ki}}=\mathrm{r}_{\mathrm{ij}} \mathrm{z}_{\mathrm{kj}}$. Рассматривается пригодность смысла z-переменной, $\mathrm{a}$ не ее значения (их $\mathrm{m}=20$ штук). Смысл $\mathrm{z}$ переменной заключается, как правило, в ее имени, приводимом дополнительно в описаниях данных. Но в обратной задаче моделирования (определения имен по значениям «весов») для выявления смысла и назначения имени используем $[9,10]$ не значение zпеременной, а используется «вес» при ее значении. Модельная матрица $\mathrm{C}^{+}{ }_{n n}$ из модели ОС36 содержит те самые значения весомых «весов» и пренебрежимо малых «весов» «как раз там, где нужно». Это является еще одним шагом к имитации реальности при моделировании матрицы $\mathrm{C}^{+}{ }_{n n}$ псевдособственных векторов- основного источника цифровых знаний [6,12-15].

В спектральных задачах ОС32, ОС33 [6], ОС34, ОС35 предполагается наличие доминирующих элементов. Такое свойство спектра присуще для спектра корреляционной матрицы $\mathrm{R}_{\mathrm{nn}}$ реальной многомерной выборки. В этих моделях в матрице $\mathrm{C}_{\mathrm{nn}}$, (состоящей из столбцов значений компонент собственных векторов), собственные векторы $\mathbf{c}_{\mathbf{j}}=\left(\mathrm{c}_{1 \mathrm{j}}, \mathrm{c}_{2 \mathrm{j}} \ldots \mathrm{c}_{\mathrm{nj}}\right)^{\mathrm{T}}$, взаимно ортогональны $\mathbf{c}_{\mathrm{j}}^{\mathrm{T}} \mathbf{c}_{\mathrm{j}}=0$, при $\mathrm{i} \neq \mathrm{j}$, и имеют единичную дину $\mathbf{c}_{\mathrm{j}}^{\mathrm{T}} \mathbf{c}_{\mathrm{j}}=1$, а в модельной матрице $\mathrm{C}^{+}{ }_{\mathrm{nn}}$ не требуется выполнения условия равенства единице длин всех векторов $\mathbf{c}_{\mathrm{j}}{ }^{+}=\left(\mathrm{c}^{+}{ }_{1 \mathrm{j}}, \mathrm{c}^{+}{ }_{2 \mathrm{j}} \ldots \mathrm{c}^{+}{ }_{\mathrm{nj}}\right)^{\mathrm{T}} \mathbf{c}_{\mathrm{j}}{ }^{+\mathrm{T}} \mathbf{c}_{\mathrm{j}}{ }^{+} \neq 1, \mathrm{j} \in\{1, \ldots, \mathrm{n}\} . \quad$ Условие ортогональны $\mathbf{c}_{\mathrm{j}}^{\mathrm{T}} \mathbf{c}_{\mathrm{j}}=0$, при $\mathrm{i} \neq \mathrm{j}$, оставим в силе. Тогда в матричном виде ограничения на модельную матрицу псевдособственных векторов примут вид: $\mathrm{C}^{+\mathrm{T}}{ }_{\mathrm{nn}} \mathrm{C}^{+}{ }_{\mathrm{nn}} \neq \mathrm{I}_{\mathrm{nn}}, \mathrm{C}^{+}{ }_{\mathrm{nn}} \mathrm{C}^{+\mathrm{T}}{ }_{\mathrm{nn}}=\mathrm{I}_{\mathrm{nn}}$. Тогда будут выполняться условия для нулевых значений коэффициентов корреляции z-переменных $\mathbf{c}_{\mathrm{i}}^{+} \Lambda^{+}{ }_{\mathrm{nn}} \mathbf{c}_{\mathrm{j}}{ }^{+\mathrm{T}}=\mathrm{r}^{+}{ }_{\mathrm{ij}}=0$. Так как $\mathrm{C}^{+}{ }_{\mathrm{nn}} \mathrm{C}^{+\mathrm{T}}{ }_{\mathrm{nn}}=\mathrm{I}_{\mathrm{nn}}, \Lambda^{+}{ }_{\mathrm{nn}}=\mathrm{I}_{\mathrm{nn}} \mathrm{Y}_{\mathrm{mn}}=\mathrm{U}_{\mathrm{mn}} \Lambda^{(1 / 2)+}{ }_{\mathrm{nn}}=\mathrm{U}_{\mathrm{mn}} \mathrm{I}^{1 / 2}{ }_{\mathrm{nn}}=\mathrm{U}_{\mathrm{mn}}$, $\mathrm{Z}_{\mathrm{mn}}=\mathrm{Y}_{\mathrm{mn}} \mathrm{C}^{+\mathrm{T}}{ }_{\mathrm{nn}}=\mathrm{U}_{\mathrm{mn}} \mathrm{C}^{+\mathrm{T}}{ }_{\mathrm{nn}}$, то имеем равенство $\mathrm{R}^{+}{ }_{\mathrm{nn}}=(1 / \mathrm{m}) \mathrm{Z}_{\mathrm{mn}}{ }^{\mathrm{T}} \mathrm{Z}_{\mathrm{mn}}=(1 / \mathrm{m})\left[\mathrm{U}_{\mathrm{mn}} \mathrm{C}^{+\mathrm{T}}{ }_{\mathrm{nn}}\right]^{\mathrm{T}}\left[\mathrm{U}_{\mathrm{mn}} \mathrm{C}^{+\mathrm{T}}{ }_{\mathrm{nn}}\right]=(1 / \mathrm{m})$ $\mathrm{C}^{+}{ }_{n n}\left(\mathrm{U}_{\mathrm{mn}}{ }^{\mathrm{T}}\left[\mathrm{U}_{\mathrm{mn}}\right) \mathrm{C}^{+\mathrm{T}}{ }_{\mathrm{nn}}\right]=\mathrm{C}^{+}{ }_{\mathrm{nn}} \mathrm{C}^{+\mathrm{T}}{ }_{\mathrm{nn}}=\mathrm{I}_{\mathrm{nn}}$. Равенство $\mathrm{R}^{+}{ }_{\mathrm{nn}}=$ $\mathrm{I}_{\mathrm{nn}}$ преобразует основное равенство ПС3 $\mathrm{R}^{+}{ }_{n n} \mathrm{C}^{+}{ }_{n n}=\mathrm{C}^{+}{ }_{n n} \Lambda^{+}{ }_{n n}$ в равенство $\mathrm{C}^{+}{ }_{n n}=\mathrm{C}^{+}{ }_{n n}$.

Геометрическая интерпретация равенств $\mathrm{C}^{+\mathrm{T}}{ }_{n n} \mathrm{C}^{+}{ }_{n n} \neq \mathrm{I}_{\mathrm{nn}}, \mathrm{C}^{+}{ }_{n n} \mathrm{C}^{+\mathrm{T}}{ }_{n n}=\mathrm{I}_{\mathrm{nn}}$, следующая: облако точек $\left(\mathrm{Z}_{\mathrm{i} 1}, \ldots, \mathrm{Z}_{\text {in }}\right), \mathrm{i}=1, \ldots, \mathrm{m}, \quad$ сосредоточено в $\mathrm{n}$-мерном шаре с радиусом, длина которого пропорциональна 1. Они получены путем умножения векторов $\left(\mathrm{y}_{\mathrm{i} 1}, \ldots, \mathrm{y}_{\mathrm{in}}\right)$ справа на матрицу $\mathrm{C}^{+\mathrm{T}}{ }_{\mathrm{nn}}$. Матрица $\mathrm{Y}_{\mathrm{mn}}=\mathrm{U}_{\mathrm{mn}}$ моделируется отдельно и независимо от матриц $\mathrm{C}^{+}{ }_{n n}$ и $\Lambda^{+}{ }_{n n}$. Точки $\left(\mathrm{y}_{\mathrm{i} 1}, \ldots, \mathrm{y}_{\mathrm{in}}\right), \mathrm{i}=1, \ldots, \mathrm{m}$, по построению находятся в шаре с радиусом, длина которого пропорциональна 1.

\section{Матрица значений коэффициентов комбинационной пропорциональности при значениях изменчивостей $\mathrm{z}$-переменных}

Известно, что коэффициент корреляции Пирсона показывает (через свое значение $\mathrm{r}_{12}$ ) насколько выражена пропорциональная изменчивость двух переменных $\mathrm{z}_{1}$ и $\mathrm{z}_{2}: \mathrm{z}_{\mathrm{k} 1}=\mathrm{r}_{12} \mathrm{Z}_{\mathrm{k} 2}$, $1 \leq \mathrm{r}_{12} \leq 1$, при всех $\mathrm{k}=1, \ldots, \mathrm{m}$. Значение $\mathrm{r}_{12}$ таково, что вектор-столбец $\left(\mathrm{z}_{11}, \ldots, \mathrm{Z}_{1 \mathrm{~m}}\right)^{\mathrm{T}}$, значений переменной $\mathrm{z}_{1}$ 


\begin{tabular}{|c|c|c|c|c|c|c|}
\hline \multirow{4}{*}{ Impact Factor: } & ISRA (India) & $=3.117$ & SIS (USA) & $=0.912$ & ICV (Poland) & $=6.630$ \\
\hline & ISI (Dubai, UAE & $=0.829$ & РИНЦ (Russia) & $=0.156$ & PIF (India) & $=1.940$ \\
\hline & GIF (Australia) & $=0.564$ & ESJI (KZ) & $=8.716$ & IBI (India) & $=4.260$ \\
\hline & JIF & $=1.500$ & SJIF (Morocco) & $=5.667$ & OAJI (USA) & $=0.350$ \\
\hline
\end{tabular}

равен вектор-столбцу $\left(\mathrm{r}_{12} \mathrm{Z}_{\mathrm{i} 2}, \ldots, \mathrm{r}_{12} \mathrm{Z}_{\mathrm{m} 2}\right)^{\mathrm{T}}$ значений переменной $\left(\mathrm{r}_{12}\right) \mathrm{z}_{2}$. Коэффициенты $(\mathrm{z}, \mathrm{z})$-корреляции $\mathrm{r}_{\mathrm{ij}}=\operatorname{corr}\left(\mathrm{z}_{\mathrm{i}}, \mathrm{z}_{\mathrm{j}}\right)$ образуют симметрическую матрицу $\mathrm{R}_{\mathrm{nn}}=(1 / \mathrm{m}) \mathrm{Z}_{\mathrm{mn}}^{\mathrm{T}} \mathrm{Z}_{\mathrm{mn}}$, а коэффициенты $(\mathrm{y}, \mathrm{z})$-корреляции $\mathrm{c}_{\mathrm{ij}}=\operatorname{corr}\left(\mathrm{z}_{\mathrm{i}}, \mathrm{y}_{\mathrm{j}}\right)$ образуют несимметрическую матрицу $\mathrm{C}_{\mathrm{nn}}=(1 / \mathrm{m}) \mathrm{Z}_{\mathrm{mn}}^{\mathrm{T}}\left[\mathrm{Y}_{\mathrm{mn}} \Lambda^{(-1)}\right]$, элементы которой показывают (через свои значения $\mathrm{c}_{\mathrm{ij}}, \mathrm{i}=1, \ldots, \mathrm{n}$, $\mathrm{j}=1, \ldots, \mathrm{n}$ ) насколько выражена непропорциональная изменчивость двух переменных: $\mathrm{y}_{\mathrm{i}}$ и $\mathrm{z}_{\mathrm{j}}$. Здесь одна переменная равна линейной комбинации $\mathrm{n} \mathrm{z}$ переменных: $\mathrm{y}_{\mathrm{ij}}=\mathrm{z}_{\mathrm{i} 1} \mathrm{c}_{1 \mathrm{j}}+\mathrm{z}_{\mathrm{i} 2} \mathrm{c}_{2 \mathrm{j}}+\ldots+\mathrm{z}_{\mathrm{in}} \mathrm{c}_{\mathrm{nj}}$, (не является стандартизованной) другая переменная (zпеременная $\left.\mathrm{z}_{\mathrm{i}}\right)$ в формуле $\mathrm{c}_{\mathrm{ij}}=\operatorname{corr}\left(\mathrm{z}_{\mathrm{i}}, \mathrm{y}_{\mathrm{j}}\right)$ является стандартизованной. Только после стандартизации одной из них: $\mathrm{y}_{\mathrm{i}} / \lambda_{\mathrm{j}}$ и $\mathrm{z}_{\mathrm{j}}(\mathrm{y}, \mathrm{z})$-корреляция между ними$\mathrm{c}_{\mathrm{ij}}=\operatorname{corr}\left(\mathrm{z}_{\mathrm{i}}, \mathrm{y}_{\mathrm{j}}\right)$ имеет тот смысл, который соответствует его определению. Несимметрическая матрица $\mathrm{C}_{\mathrm{nn}}=(1 / \mathrm{m}) \mathrm{Z}_{\mathrm{mn}}^{\mathrm{T}}\left[\mathrm{Y}_{\mathrm{mn}} \Lambda^{(-1)}\right]$ «весов» с коэффициентами непропорциональных изменчивостей содержит значимые (значение элемента матрицы $\mathrm{C}_{\mathrm{nn}}$ имеет весомый «вес») и незначимые элементы (значение элемента матрицы $\mathrm{C}_{\mathrm{nn}}$ не имеет «веса», удовлетворяющего критерию весомости). Значимый элемент называем «вес»и и используем его в качестве индикатора наличия содержательного смысла у z-переменной, значение $\mathrm{z}_{\mathrm{i} 2}$ которой умножается на значение значимого элемента: $Z_{i 1} c_{1 j}$. Весомый «вес» не единствен. Значения «весов» и весов из матриц $\mathrm{C}^{(\ell)}{ }_{n n}$ с номерами $\ell=1, \ldots, \propto$, для фиксированной матрицы $\Lambda^{+}{ }_{\text {nn }}$ могут моделироваться при решении ОС31 [3], OC32, ОС33 [6-8], OC34, ОС35, ОС36.

Коэффициент корреляции $\mathrm{r}_{12}$ является коэффициентом пропорциональности между значением изменчивости $\mathrm{Z}_{\mathrm{k} 1}$ и значением «изменчивости» $\mathrm{Z}_{\mathrm{k} 2}[1-5]: \quad \mathrm{Z}_{\mathrm{k} 1}=\mathrm{r}_{12} \mathrm{Z}_{\mathrm{k} 2}, \mathrm{k}=1, \ldots, \mathrm{m} . \mathrm{B}$ когнитивных моделях извлечения знаний удобно вместо термина «коэффициент корреляции» $\left(\mathrm{r}_{12}\right)$ применять термин «коэффициент пропорциональности между двумя значениями двух $\mathrm{z}$-переменных». Чем «весомее» значение $\mathrm{r}_{12}$, тем лучше.

Коэффициент корреляции между переменной и $\mathrm{z}$-переменной $\mathrm{c}_{\mathrm{ij}}=\operatorname{corr}\left(\mathrm{z}_{\mathrm{i}}, \mathrm{y}_{\mathrm{j}}\right)$ является коэффициентом не пропорциональности, а совместно производящим коэффициентом комбинации пар коэффициентов $\left(c_{i j}, c_{1 j}\right),\left(c_{i j}, c_{2 j}\right), \ldots,\left(c_{i j}, c_{n j}\right)$ пропорциональностей. Первый член $\mathrm{c}_{\mathrm{ij}}$ (c индексом $(\mathrm{i}, \mathrm{j})$ ) пары пропорционален значению $\mathrm{z}_{\mathrm{j}}$ (c индексом (i)) изменчивости (i-ой z-переменной $\mathrm{z}_{\mathrm{j}}$ ), 2-ой член $\mathrm{c}_{1 \mathrm{j}}$ (c индексом $(1, \mathrm{j}))$ пары пропорционален значению $\mathrm{Z}_{\mathrm{i}}$ (с индексом $(\mathrm{i}, 1))$ изменчивости переменной $\mathrm{z}_{1}(1-\mathrm{oй}$ $\mathrm{z}$-переменной $\mathrm{z}_{1}$, являющейся частью j-ой $\mathrm{y}$ переменной $\mathrm{y}_{\mathrm{j}}$ ) и значениям изменчивостей всех остальных $\mathrm{z}$-переменных $\mathrm{z}_{2}, \ldots \mathrm{z}_{\mathrm{n}}$. Соответствие индексов i-ой z- переменной и j-ой у- переменной, образуют пару $(\mathrm{i}, \mathrm{j})$ элемента $\mathrm{c}_{\mathrm{ij}}$. Этой паре индексов соответствует комбинация пар индексов значений изменчивостей всех $\mathrm{z}$-переменных $\mathrm{z}_{1}, \ldots \mathrm{z}_{\mathrm{n}}$ : $[(i, j),(1, j)]=>[(i, 1),(j)] \oplus[(i, 2),(j)] \oplus \ldots \oplus[(i, n),(j)]$.

Интерпретацию значения коэффициента комбинационной пропорциональности следующая. $\mathrm{B}$ переменной $\mathrm{y}_{\mathrm{i}}$ содержится линейная комбинация $\mathrm{z}$-переменных $\mathrm{z}_{1}, \ldots, \mathrm{z}_{\mathrm{n}}\left(\mathrm{y}_{\mathrm{ij}}=\mathrm{z}_{\mathrm{i} 1} \mathrm{c}_{1 \mathrm{j}}+\mathrm{z}_{\mathrm{i} 2} \mathrm{c}_{2 \mathrm{j}}+\ldots+\mathrm{z}_{\mathrm{in}} \mathrm{c}_{\mathrm{nj}}\right)$. В каждом ее слагаемом имеется свой «вес» из перечня $\mathrm{c}_{1 \mathrm{j}}, \mathrm{c}_{2 \mathrm{j}}, \ldots, \mathrm{c}_{\mathrm{nj}}$ (весомый или не весомый), влияющий на «вес» $\mathrm{c}_{\mathrm{ij}}$ при $\mathrm{z}$-переменной $\mathrm{z}_{1}$. Таким образом из формулы $\mathrm{c}_{\mathrm{ij}}=\operatorname{corr}\left(\mathrm{z}_{\mathrm{i}}, \mathrm{y}_{\mathrm{j}}\right)$ видим, что на одно значение «веса» умножаются все значения элементов одной строки матрицы $\mathrm{C}_{\mathrm{nn}}$, появляются составляющие, отсутствующие в пропорциональной зависимостинелинейные искажения. Это вывод следует из равенства $\mathrm{C}^{+}{ }_{\mathrm{nn}} \mathrm{C}^{+\mathrm{T}}{ }_{\mathrm{nn}}=\mathrm{I}_{\mathrm{nn}}$, используемого при вычислении матрицы «весов» $\mathrm{C}^{+}{ }_{\mathrm{nn}}=$ $(1 / \mathrm{m}) \mathrm{Z}^{\mathrm{T}}{ }_{\mathrm{mn}}\left[\mathrm{Y}_{\mathrm{mn}} \Lambda^{(-1)}\right]$, в ОС 36 , где для матрицы zпеременных $Z_{\mathrm{mn}}$ и для матрицы $\mathrm{y}$-переменных верны равенства $\mathrm{Z}_{\mathrm{mn}}=\mathrm{U}_{\mathrm{mn}} \mathrm{C}^{+\mathrm{T}}{ }_{\mathrm{nn}}, \mathrm{Y}_{\mathrm{mn}}=\mathrm{U}_{\mathrm{mn}}$.

Матрица значений коэффициентов комбинационной пропорциональности при значениях изменчивостей $\mathrm{z}$-переменных является одновременно матрицей значений коэффициентов комбинационной пропорциональности при значениях изменчивостей $\mathrm{u}$-переменных. Этот вывод следует из равенств из модели ОС 36 :

$\mathrm{Y}_{\mathrm{mn}}=\mathrm{U}_{\mathrm{mn}}, \mathrm{Z}_{\mathrm{mn}}=\mathrm{U}_{\mathrm{mn}} \mathrm{C}^{+\mathrm{T}}{ }_{\mathrm{nn}}$,

$\mathrm{C}^{+}{ }_{n n}=(1 / \mathrm{m})\left[\mathrm{U}_{\mathrm{mn}} \mathrm{C}^{+\mathrm{T}}{ }_{\mathrm{nn}}\right]^{\mathrm{T}}{ }_{\mathrm{mn}}\left[\mathrm{U}_{\mathrm{mn}}\right]=(1 / \mathrm{m}) \mathrm{C}^{+}{ }_{\mathrm{nn}}\left[\mathrm{U}^{\mathrm{T}}{ }_{\mathrm{mn}} \mathrm{U}_{\mathrm{mn}}\right]=$ $\mathrm{C}^{+}{ }_{n n}(1 / \mathrm{m})\left[\mathrm{U}^{\mathrm{T}}{ }_{\mathrm{mn}} \mathrm{U}_{\mathrm{mn}}\right]=\mathrm{C}^{+}{ }_{\mathrm{nn}} \mathrm{I}_{\mathrm{nn}}=\mathrm{C}^{+}{ }_{\mathrm{nn}}$.

Особо подчеркнем, что одно значение «веса» умножаются на все значения элементов одной строки матрицы $\mathrm{C}_{\mathrm{n} n}$, но со своими коэффициентами пропорциональности. Эти коэффициенты пропорциональности равны значениям «изменчивостей» из матрицы $Z_{\mathrm{mn}}$. Эту пропорциональность мы назвали «комбинационной пропорциональностью», отличной от прямой пропорциональности между значением изменчивости одной $\mathrm{z}$-переменной $\left(\mathrm{z}_{\mathrm{k} 1}\right)$ и значением «изменчивости» другой $\mathrm{Z}$-переменной $\left(\mathrm{z}_{\mathrm{k} 2}\right)$.Термин «комбинационная» имеет смысл комбинации «пропорциональностей» $\mathrm{Z}$-переменных $\mathrm{z}_{1}, \ldots, \mathrm{z}_{\mathrm{n}}$. При выполнении равенств $\mathrm{c}_{1 \mathrm{j}}=\mathrm{c}_{2 \mathrm{j}}=\ldots=\mathrm{c}_{\mathrm{nj}}=\mathrm{c}_{\mathrm{j}}$ в слагаемых $\mathrm{Z}_{\mathrm{i} 1} \mathrm{c}_{1 \mathrm{j}}, \quad \mathrm{Z}_{\mathrm{i} 2} \mathrm{c}_{2 \mathrm{j}}, \ldots, \mathrm{Z}_{\mathrm{in}} \mathrm{c}_{\mathrm{nj}}$ выполняется равенство $\mathrm{y}_{\mathrm{ij}}=\mathrm{c}_{\mathrm{j}}\left(\mathrm{z}_{\mathrm{i} 1}+\mathrm{z}_{\mathrm{i} 2}+\ldots+\mathrm{z}_{\text {in }}\right)$ и имеем пропорциональную связь между значением изменчивости у-переменной $\mathrm{y}_{\mathrm{j}}$ и значением «изменчивости» переменной $\left(\mathrm{z}_{1}+\mathrm{z}_{2}+\ldots+\mathrm{z}_{\mathrm{n}}\right)$.

При выполнении равенства $\mathrm{Z}_{1}=\mathrm{Z}_{\mathrm{i} 1}=\mathrm{Z}_{\mathrm{i} 2}=\ldots=\mathrm{Z}_{\text {in }}$ в слагаемых $\mathrm{Z}_{\mathrm{i} 1} \mathrm{c}_{1 \mathrm{j}}, \quad \mathrm{Z}_{\mathrm{i} 2} \mathrm{c}_{2 \mathrm{j}}, \ldots, \mathrm{Z}_{\mathrm{in}} \mathrm{c}_{\mathrm{nj}} \quad$ выполняется равенство $\mathrm{y}_{\mathrm{j}}=\mathrm{z}_{1}\left(\mathrm{c}_{1 \mathrm{j}}+\mathrm{c}_{2 \mathrm{j}}+\ldots+\mathrm{c}_{\mathrm{nj}}\right)=\mathrm{z}_{1}\left(\mathrm{c}_{\mathrm{j}}\right)$ и могли бы иметь пропорциональную связь между значением изменчивости $y_{j} \mathrm{j}$-ой $\mathrm{y}$-переменной $\mathrm{y}_{\mathrm{j}}$ и значением «изменчивости» одной «составной» переменной $\mathrm{Z}_{1}$. Но выполнение равенств $\mathrm{Z}_{1}=\mathrm{Z}_{\mathrm{i} 1}=\mathrm{Z}_{\mathrm{i} 2}=\ldots=\mathrm{Z}_{\text {in }}$ 


\begin{tabular}{|c|c|c|c|c|c|c|}
\hline \multirow{4}{*}{ Impact Factor: } & ISRA (India) & $=3.117$ & SIS (USA) & $=0.912$ & ICV (Poland) & $=6.630$ \\
\hline & ISI (Dubai, UAE & $=0.829$ & РИНЦ (Russia) & $=0.156$ & PIF (India) & $=1.940$ \\
\hline & GIF (Australia) & $=0.564$ & ESJI (KZ) & $=8.716$ & IBI (India) & $=4.260$ \\
\hline & JIF & $=1.500$ & SJIF (Morocco) & $=5.667$ & OAJI (USA) & $=0.350$ \\
\hline
\end{tabular}

невозможны, поэтому изменчивости между упеременной $\mathrm{y}_{\mathrm{j}}$ и линейной комбинацией из $\mathrm{n} \mathrm{z}$ переменных $\mathrm{z}_{1}, \ldots, \mathrm{z}_{\mathrm{n}}$ не будут иметь пропорциональную связь, а имеют, как показано выше, «комбинационную пропорциональность».

Теперь становится более ясной концепция применений [16] когнитивного графа узла и когнитивных карт, основанных на визуализации и осмыслении пар $\left(\lambda_{\mathrm{j}}, \mathbf{c}_{\mathbf{j}}\right)$ с доминирующими элементами $\lambda_{\mathrm{j}}$. Среди компонент ј-го собственного вектора $\mathbf{c}_{\mathbf{j}}=\left(\mathrm{c}_{1 \mathrm{j}}, \mathrm{c}_{2 \mathrm{j}} \ldots \mathrm{c}_{\mathrm{nj}}\right)^{\mathrm{T}}$ находятся весомые значения компонент $c_{\mathrm{kj}}$, по абсолютной величине удовлетворяющие критерию $\operatorname{abs}\left(\mathrm{c}_{\mathrm{kj}}\right) \geq \mathrm{c}_{0}$, $\mathrm{k} \in\{1, \ldots, \mathrm{n}\}$. Они являются индикаторами наличия содержательных смыслов у имен $\mathrm{z}$-переменных (значения которых умножены на значение «веса» $\mathrm{c}_{\mathrm{kj}}$ ), а линейная комбинация зависимых или не зависимых смыслов $\ell<\mathrm{n}$ z-переменных «равна» смыслу у-переменной. Настоящая статья может служить обоснованием концепции применений [910,12-16] когнитивного графа узла и когнитивных карт при извлечении цифровых знаний из таблиц объект-свойства при применении (H-Z)-модели главных компонент [1-2].

«Комбинационная пропорциональность» между переменными $\mathrm{y}_{\mathrm{i}}, \quad$ и $\mathrm{z}_{\mathrm{j}}$ не равна «комбинационной пропорциональности» между переменными $\mathrm{z}_{\mathrm{i}}$, и $\mathrm{y}_{\mathrm{j}}$. так как матрица $\mathrm{C}_{\mathrm{nn}}-$ не симметрична, матрица $\mathrm{R}_{\mathrm{nn}}$ - симметрична. Матрица $\mathrm{C}^{+}{ }_{n n}$ при когнитивном моделировании интерпретируется как матрица значений коэффициентов комбинационной пропорциональности при значениях изменчивостей z-переменных. В нашей модели она модельная и обладает желаемыми свойствами, будучи преобразована процедурой Solver из «реальной» матрицы собственных векторов. Она получена при решении одной из Оптимизационных Задач. Модель ОС36 имеет бесконечное множество решений.

Независимость

смыслов некоррелированных переменных

При когнитивном восприятии смыслов слагаемых $\mathrm{z}_{\mathrm{i} 1} \mathrm{c}_{1 \mathrm{j}}, \quad \mathrm{z}_{\mathrm{i} 2} \mathrm{c}_{2 \mathrm{j}}, \ldots, \mathrm{z}_{\mathrm{in}} \mathrm{c}_{\mathrm{nj}}$ из их суммы $\mathrm{y}_{\mathrm{ij}}=\mathrm{z}_{\mathrm{i} 1} \mathrm{c}_{1 \mathrm{j}}+\mathrm{z}_{\mathrm{i} 2} \mathrm{c}_{2 \mathrm{j}}+\ldots+\mathrm{z}_{\mathrm{in}} \mathrm{c}_{\mathrm{nj}}$ выделяют только слагаемые с значимыми «весами» $\mathrm{c}_{1 \mathrm{j}}, \mathrm{c}_{2 \mathrm{j}}, \ldots, \mathrm{c}_{\mathrm{nj}}$ [6-10]. Значения $\mathrm{Z}_{\mathrm{i} 1}, \mathrm{Z}_{\mathrm{i} 2}, \ldots, \mathrm{Z}_{\text {in }}$ показывают «изменчивости» «весов» $\mathrm{c}_{1 \mathrm{j}}, \mathrm{c}_{2 \mathrm{j}}, \ldots, \mathrm{c}_{\mathrm{nj}}: \mathrm{Z}_{\mathrm{i} 1} \mathrm{c}_{1 \mathrm{j}}, \mathrm{Z}_{\mathrm{i} 2} \mathrm{c}_{2 \mathrm{j}}, \ldots, \mathrm{Z}_{\mathrm{in}} \mathrm{c}_{\mathrm{nj}}$, а их сумма $\mathrm{Z}_{\mathrm{i} 1} \mathrm{c}_{1 \mathrm{j}}+\mathrm{Z}_{\mathrm{i} 2}$ $c_{2 j}+\ldots+Z_{i n} c_{n j}$ интерпретируется как одна сумма «изменчивостей» «весов». Сумма значимых «весов» является весомым (значимым) «весом» для соответствующей $\mathrm{y}$-переменной из рассматриваемой пары $\left(\lambda_{j}, \mathbf{c}_{\mathrm{j}}\right)$. Иные пары $\left(\lambda_{\mathrm{j}}, \mathbf{c}_{\mathrm{i}}\right)$, где $\mathrm{i} \neq \mathrm{j}$, не рассматриваются, номер собственного числа $\mathrm{j}$ должен совпадать с номером собственного вектора. Количество обладающих весомыми (значимыми) «весами» $\mathrm{y}$-переменных равно $\ell$. В модельной матрице количество значимых «весов» у ј-ой упеременной не зависит от значения $\lambda_{\mathrm{j}}-\mathrm{y} \quad \mathrm{y}$ переменной №1 может быть меньше значимых «весов», чем у у-переменной №4. В то время как вариабельность переменной №1 больше, чем вариабельность переменной №4. Переменная $\mathrm{z}_{\mathrm{j}}$ имеет смысл, если она обладает значимым [6,12-15] «весом» $\mathrm{c}_{1 \mathrm{j}}$, присутствующим с любым значением коэффициента «изменчивости» $\mathrm{Z}_{\mathrm{kj}}$ при $\mathrm{Z}_{\mathrm{i} 1} \mathrm{c}_{1 \mathrm{j}}$. Первичен значимый «вес» $\mathrm{c}_{1 \mathrm{j}}$, вторична величина коэффициента «изменчивости» $\mathrm{z}_{\mathrm{kj}}$. Количество таких произведений с значимыми [6,12-15] «весами» при $\mathrm{z}$-переменных в линейной комбинации вида $\mathrm{y}_{\mathrm{ij}}=\mathrm{z}_{\mathrm{i} 1} \mathrm{c}_{1 \mathrm{j}}+\mathrm{z}_{\mathrm{i} 2} \mathrm{c}_{2 \mathrm{j}}+\ldots+\mathrm{z}_{\mathrm{in}} \mathrm{c}_{\mathrm{nj}}$ может быть равно $\ell=1,2,3,4$.

Такая трансформация ролей терминов необходима для озвучивания смысла у-переменной, равной линейной комбинации смыслов у переменной $\mathrm{y}_{\mathrm{ij}}=\mathrm{z}_{\mathrm{i} 1} \mathrm{c}_{1 \mathrm{j}}+\mathrm{z}_{\mathrm{i} 2} \mathrm{c}_{2 \mathrm{j}} \ldots+\mathrm{z}_{\mathrm{in}} \mathrm{c}_{\mathrm{nj}}$, где только $\ell$ «весов» удовлетворяют критериям «существенности», «значимости». Чем больше число $\ell$, тем труднее формулируется смысл линейной комбинации вида $\mathrm{y}_{\mathrm{ij}}=\mathrm{z}_{\mathrm{i} 1} \mathrm{c}_{1 \mathrm{j}}+\mathrm{z}_{\mathrm{i} 2} \mathrm{c}_{2 \mathrm{j}}+\ldots+\mathrm{z}_{\mathrm{in}} \mathrm{c}_{\mathrm{nj}}$ (валидной переменной). Такие случаи приведены в работах [12-15]. В [12-15] приведены когнитивные модели выявления смысла суммы значимых (весомых) переменных для известных смыслов $\ell$ штук z-переменных. В [6] приведены когнитивные модели выявления неизвестных смыслов $\ell$ zпеременных, входящих в у-переменную, состоящей из линейной комбинации значимых (весомых) zпеременных. В этих 2-х классах когнитивных моделей рассматриваются взаимно обратные когнитивные задачи: первая - задача извлечения цифрового знания из цифровых данных, вторая задача цифровизации z-переменных при известных смыслах $\ell$ валидных показателей (у-переменных). Матрица значений коэффициентов комбинационной пропорциональности при значениях изменчивостей $\mathrm{z}$-переменных превращается в матрицу значений коэффициентов комбинационной пропорциональности при значениях изменчивостей $\mathrm{u}-$ переменных. В связи с этим возникает вопрос существования независимых смыслов для некоррелированных u-переменных. Если есть независимые смысли у реальных валидных показателей, к которым мы поставили в соответствие некоррелированные у-переменные, то существуют модельные зависимые $\mathrm{z}$-переменные. Вопрос существования независимых смыслов у некоррелированных $\mathrm{z}$-переменных (разбиение одного независимого смысла на несколько независимых смыслов) мы не рассматриваем, для него требуется отдельное исследование.

В ПМ ГК [1] первична выборка $Z_{m n}$ значений коррелированных z-переменных. Она является 


\begin{tabular}{|c|c|c|c|c|c|c|}
\hline \multirow{4}{*}{ Impact Factor: } & ISRA (India) & $=3.117$ & SIS (USA) & $=0.912$ & ICV (Poland) & $=6.630$ \\
\hline & ISI (Dubai, UAE & $=0.829$ & РИНЦ (Russia) & $=0.156$ & PIF (India) & $=1.940$ \\
\hline & GIF (Australia) & $=0.564$ & ESJI (KZ) & $=8.716$ & IBI (India) & $=4.260$ \\
\hline & JIF & $=1.500$ & SJIF (Morocco) & $=5.667$ & OAJI (USA) & $=0.350$ \\
\hline
\end{tabular}

первичной и определяющей для далее вычисляемых других матриц $\mathrm{R}_{\mathrm{nn}}, \mathrm{C}_{\mathrm{nn}}, \Lambda_{\mathrm{nn}}, \mathrm{Y}$ mn из ПМ ГК: $\mathrm{Z}_{\mathrm{mn}}=>\left(\mathrm{R}_{\mathrm{nn}}, \mathrm{C}_{\mathrm{nn}}, \Lambda_{\mathrm{nn}}, \mathrm{Y}_{\mathrm{mn}}\right)$.

B Обратных Задачах нам необходимо иметь данные, соответствующие независимым смыслам zпеременных. Логично выявлять у коррелированных z-переменных зависимые смыслы, а у некоррелированных z-переменных - независимые смыслы. Для моделирования выборки $\mathrm{Z}_{\mathrm{mn}}$ значений некоррелированных $\mathrm{z}$-переменных разработаем математическую модель - ниже она разработана в результате решения новой Обратной Спектральной Задачи - ОС36.

В результате решения ОСЗ6 получим модельную матрицу псевдо собственных векторов $\mathrm{C}^{+}{ }_{n n}$. Эту матрицу в когнитивных моделях извлечения цифровых знаний, в когнитивных алгоритмах придания смыслов удобно называть матрицей значений коэффициентов комбинационной пропорциональности при значениях изменчивостей некоррелированных Zпеременных. А матрицу $\mathrm{Z}_{\mathrm{mn}}$ - матрицей значений коэффициентов изменчивостей.

Все матрицы из модели ОС 36 удовлетворяют равенствам: $\mathrm{C}^{+\mathrm{T}}{ }_{\mathrm{nn}} \mathrm{C}^{+}{ }_{\mathrm{nn}} \neq \mathrm{I}_{\mathrm{nn}}, \mathrm{C}^{+}{ }_{\mathrm{nn}} \mathrm{C}^{+\mathrm{T}}{ }_{\mathrm{nn}}=\mathrm{I}_{\mathrm{nn}}, \Lambda^{+}{ }_{\mathrm{nn}},=\mathrm{I}_{\mathrm{nn}}$, $\mathrm{Y}_{\mathrm{mn}}=\mathrm{U}_{\mathrm{nn}}, \mathrm{Z}_{\mathrm{mn}}=\mathrm{U}_{\mathrm{nn}}, \mathrm{R}_{\mathrm{nn}}=\mathrm{I}_{\mathrm{nn}}$, служат допущениями, но доминирующие роли при когнитивном извлечении цифровых знаний из многомерной выборки $\mathrm{Z}_{\mathrm{mn}}=\mathrm{U}_{\mathrm{mn}}\left[\mathrm{C}^{+}{ }_{\mathrm{nn}}\right]^{\mathrm{T}}$ имеет модельную матрица «весов» $\mathrm{C}^{+}{ }_{\mathrm{nn}}$. Ниже приведем подтверждающие факты.

\section{Матрица собственных векторов $\mathrm{C}_{n n}$ - входной объект в моделях ОСЗ}

В связи с модификациями и приложениями моделей [6-10] и их новых задач становится актуальным исследование зависимости $\mathrm{C}_{\mathrm{nn}}$ от $\Lambda_{\mathrm{nn}}$. Актуализируем в новых Обратных Спектральных Задачах вопрос: что первично $\mathrm{C}_{\mathrm{nn}}$ или $\Lambda_{\mathrm{nn}}$ ? Иначе говоря суть вопроса в том, что мы должны иметь в качестве входного объекта в моделях ОС3? Ответ: это зависит от решаемой обратной задачи. Если мы решаем задачу моделирования многомерной выборки, адекватной многомерной реальной выборке, то входным объектом может быть пара $\left(\mathrm{C}_{\mathrm{nn}}, \Lambda_{\mathrm{nn}}\right)$.

Если мы решаем задачу моделирования многомерной выборки, содержащей цифровые знания, извлекаемые с применением когнитивного моделирования [6-9], то входным объектом может быть матрица псевдособственных векторов $\mathrm{C}^{+}{ }_{\mathrm{n}}$.

Известно, что в модели ОС31: $\Lambda_{\mathrm{nn}}=>\left(\mathrm{C}^{(\ell)}{ }_{\mathrm{nn}}, \mathrm{R}^{(\ell)}{ }_{\mathrm{nn}}\right), \ell=1, \ldots, \mathrm{k}_{\ell}<\infty$, собственные числа меняются при переходе к другой системе собственных векторов (к другому базису) $\mathrm{C}^{(\ell)}{ }_{\mathrm{nn}}=\left[\mathbf{c}_{1}\left|\mathbf{c}_{2}\right| \ldots \mid \mathbf{c}_{\mathbf{n}}\right]$, где $\mathbf{c}_{\mathrm{j}}^{\mathrm{T}} \mathbf{c}_{\mathrm{j}}=0$, при $\mathrm{i} \neq \mathrm{j}, \quad \mathbf{c}_{\mathrm{j}}^{\mathrm{T}} \mathbf{c}_{\mathrm{j}}=1$, $\mathrm{j}=1, \ldots, n ; \mathrm{i}=1, \ldots, n$. В паре матриц $\left(\mathrm{C}_{\mathrm{nn}}, \Lambda_{\mathrm{nn}}\right)$ ее пары $\left(\lambda_{\mathrm{j}}, \mathbf{c}_{\mathrm{j}}\right)$ собственных объектов таковы, что для неизменного значения собственного числа $\lambda_{\mathrm{j}}$, моделируются бесконечное количество собственных векторов $\mathbf{c}_{\mathbf{j}}=\left(\mathrm{c}_{1 \mathrm{j}}, \mathrm{c}_{2 \mathrm{j}} \ldots \mathrm{c}_{\mathrm{nj}}\right)^{\mathrm{T}}, \mathrm{j}=1, \ldots, \mathrm{n}$. Неизменность значений собственных чисел: либо всех (в ОС31), либо только доминирующих $\lambda_{1}, \ldots, \lambda_{\ell}$ (в ОС32, ОС 33, ОС 34, ОС35) позволяет успешно решать Оптимизационные Задачи с соответствующими допущениями на их параметры и переменные.

В ОС36 нарушим эту традицию. Будем решать OC3, в которой сперва меняем все или часть собственных векторов, а затем - собственные числа, до тех фиксированных заранее значений, которые мы считаем заданными. Иначе говоря, сперва переходим к другому базису (с заданными значениями некоторых компонент вектора), затем меняем соответствующие ему (вектору) собственные числа до заданных значений. В настоящей статье заданные значения одинаковы и равны 1.

Эти модели ОС3 (Таблица 1) применяются при моделировании матриц собственных векторов и матрицы собственных чисел. Пары этих матриц $\left(\mathrm{C}_{\mathrm{nn}}, \Lambda_{\mathrm{nn}}\right)$ приведены в Таблице 1. Отметим, что

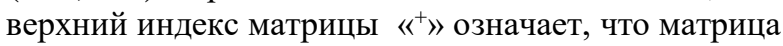
получена в результате решения ОС3, ПС3, но не ОС31. Отсутствие этого индекса у матрицы означает ее принадлежность к ПСЗ, ОС 31. Подробно опишем модель ОС36.

В публикациях по ПМСА большое значение уделялось элементам матрицы $\Lambda_{\mathrm{nn}}$ собственных чисел, ее доминирующим элементам $\lambda_{1}, \lambda_{2}, \ldots, \lambda_{\ell}$. Моделированию полного спектра $\Lambda_{\mathrm{nn}}$ с заданными значениями $\mathrm{f}_{1}, \mathrm{f}_{2}, \mathrm{f}_{3}, \mathrm{f}_{4}, \mathrm{f}_{5}, \mathrm{f}_{6}$ ее нелинейных функций $\mathrm{f}$-параметров $\mathrm{f}_{\mathrm{s}}\left(\Lambda^{+}{ }_{\mathrm{nn}}\right)=\mathrm{f}_{\mathrm{s}}, \mathrm{s}=1,2,3,4,5,6$, посвящена статья [4].

В работах [2-4] для заданной матрицы собственных чисел используется соответствующая ей матрица собственных векторов или моделируются новые матрицы собственных векторов или пары матриц $\left(\mathrm{C}^{+(\ell)}{ }_{\mathrm{nn}}, \Lambda^{+(\ell)}{ }_{\mathrm{nn}}\right)$. При этом для новой моделируемой матрицы $\mathrm{C}^{+(\ell)}{ }_{n n}$ соответственно моделируется матрицы собственных чисел вида $\Lambda^{+}{ }_{n n}=\left[\Lambda_{1} \mid \Lambda_{2}^{+}\right], \Lambda_{n n}^{+}=\left[\Lambda_{1}^{+} \mid \Lambda_{2}^{+}\right]$или $\Lambda^{+}{ }_{\mathrm{nn}}=\left[\Lambda_{1} \mid \Lambda_{2}\right]$. Новая моделируемая матрица $\mathrm{C}^{+(\ell)}{ }_{\mathrm{nn}}$ меняет либо подматрицу $\mathrm{C}^{+(\ell)}$, либо подматрицу $\mathrm{C}^{+(\ell)}{ }_{2}$.

Ниже приведены результаты и писания решений задач моделирования матрицы $\mathrm{C}^{+}{ }_{n n}, \mathrm{y}$ которой меняются либо 2 подматрицы $\mathrm{C}^{+(\ell)}{ }_{1}=$ $\left[\mathrm{C}^{+} \mid \mathrm{C}^{+}{ }_{2}\right]$ либо одна подматрица $\mathrm{C}^{+(\ell)}{ }_{2}$ : $\mathrm{C}^{+(\ell)}{ }_{\mathrm{nn}}=\left[\mathrm{C}_{1} \mid \mathrm{C}_{2}^{+}\right]$. Отличия ОС3 1, ОС3 2 и ОСЗ3 друг от друга иллюстрирует Таблица 2. 


\begin{tabular}{|c|c|c|c|c|c|c|}
\hline \multirow{4}{*}{ Impact Factor: } & ISRA (India) & $=3.117$ & SIS (USA) & $=0.912$ & ICV (Poland) & $=6.630$ \\
\hline & ISI (Dubai, UAE & $=0.829$ & РИНЦ (Russia) & $=\mathbf{0 . 1 5 6}$ & PIF (India) & $=1.940$ \\
\hline & GIF (Australia) & $=0.564$ & ESJI (KZ) & $=8.716$ & IBI (India) & $=4.260$ \\
\hline & JIF & $=1.500$ & SJIF (Morocco) & $=5.667$ & OAJI (USA) & $=0.350$ \\
\hline
\end{tabular}

Таблица 2. ОС 3, соответствующие ПСЗ: $R_{n n}=>\left(C_{n n}, \Lambda_{n n}\right)$

\begin{tabular}{|c|c|c|c|c|c|c|c|}
\hline $\begin{array}{l}\text { Номер } \\
\text { OC3 }\end{array}$ & Схема ОС3 & Собств. числа & $\begin{array}{l}\text { Изменяемые } \\
\text { соб. числа }\end{array}$ & $\begin{array}{l}\text { Собств. } \\
\text { векторы }\end{array}$ & $\begin{array}{l}\text { Выделенн } \\
\text { ые комп- } \\
\text { ты } \quad \text { соб. } \\
\text { векторов }\end{array}$ & $\begin{array}{l}\text { Изменяем } \\
\text { ые соб. } \\
\text { векторы }\end{array}$ & $\begin{array}{l}\text { Посто } \\
\text { янные } \\
\text { соб. } \\
\text { векто } \\
\text { ры }\end{array}$ \\
\hline 1 & 2 & 3 & 4 & 5 & 6 & 7 & 8 \\
\hline OC3 1 & $\begin{array}{l}\Lambda_{\mathrm{nn}}=>\left(\mathrm{C}^{(\ell)}{ }_{\mathrm{nn}}, \mathrm{R}^{(\ell)}{ }_{\mathrm{nn}}\right), \ell=1, \\
\ldots, \mathrm{k}_{\ell}<\infty,\end{array}$ & $\begin{array}{l}\Lambda_{\mathrm{nn}}=\operatorname{diag}\left(\lambda_{1}, \ldots,\right. \\
\left.\lambda_{\mathrm{n}}\right)\end{array}$ & нет & $\begin{array}{l}\mathrm{C}^{(\ell) \mathrm{T}}{ }_{\mathrm{nnn}} \mathrm{C}^{(\ell)}{ }_{\mathrm{nn}}=\mathrm{C}^{(\ell)} \\
{ }_{\mathrm{nn}} \mathrm{C}^{(\ell) \mathrm{r}} \mathrm{rn}_{\mathrm{nn}}=\mathrm{I}_{\mathrm{nn}},\end{array}$ & нет & нет & \\
\hline OC3 2 & $\begin{array}{l}{\left[\mathrm{C}^{0} \mid \mathrm{C}_{2}\right]=>\left[\mathrm{C}^{+}{ }_{1} \mid \mathrm{C}_{2}\right]} \\
=>\left(\mathrm{C}^{+}{ }_{\mathrm{nn}}, \Lambda^{+}{ }_{\mathrm{nn}}\right)\end{array}$ & $\begin{array}{l}\Lambda^{+}{ }_{\mathrm{nn}}=\operatorname{diag} \\
\left(\lambda^{+}{ }_{1}, \ldots, \lambda^{+}{ }_{\mathrm{n}}\right)\end{array}$ & $\lambda^{+}{ }_{1}, \ldots, \lambda_{n}^{+}$ & $\begin{array}{l}\mathrm{C}^{+\mathrm{T}}{ }_{n n} \mathrm{C}^{+}{ }_{n n}=\mathrm{C}^{+}{ }_{\mathrm{nn}} \mathrm{C} \\
{ }^{+\mathrm{T}}{ }_{\mathrm{nn}}=\mathrm{I}_{\mathrm{nn}}, \mathrm{C}^{+}{ }_{\mathrm{nn}} \Lambda^{+}{ }_{\mathrm{nn}} \\
\mathrm{C}^{+\mathrm{T}}{ }_{\mathrm{nn}}=\mathrm{R}^{+}{ }_{\mathrm{nn}},\end{array}$ & $\begin{array}{ll}\text { эл-ты } \\
\mathrm{C}^{+}{ }_{1}\end{array}$ & $\mathrm{C}^{+}{ }_{1}$ & $\mathrm{C}_{2}$ \\
\hline OC3 3 & $\begin{array}{c}\left(\Lambda_{\mathrm{nn}}, \mathrm{C}_{\mathrm{nn}}\right)=>\left(\Lambda_{\mathrm{nn}}, \mathrm{C}_{\mathrm{nn}}=[\mathrm{C}\right. \\
\left.{ }_{1} \mid \mathrm{C}^{0+}{ }_{2}\right]=>\left(\mathrm{C}^{+}{ }_{\mathrm{nn}}=\right. \\
\left.\left[\mathrm{C}_{1} \mid \mathrm{C}^{+}{ }_{2}\right], \Lambda^{+}{ }_{\mathrm{nn}}\right) .\end{array}$ & $\begin{array}{c}\Lambda_{\mathrm{nn}}^{+}=\operatorname{diag}\left(\lambda_{1},\right. \\
\ldots \lambda_{\ell} \\
\left.\lambda^{+}{ }_{\ell+1}, \ldots, \lambda_{\mathrm{n}}^{+}\right)\end{array}$ & $\lambda^{+} \ell+1, \ldots, \lambda_{n}^{+}$ & $\begin{array}{c}\mathrm{C}^{+\mathrm{T}}{ }_{n n} \mathrm{C}^{+}{ }_{n n}=\mathrm{C}^{+}{ }_{n n} \mathrm{C} \\
{ }^{+\mathrm{T}}{ }_{\mathrm{nn}}=\mathrm{I}_{\mathrm{nn}}, \mathrm{C}^{+}{ }_{\mathrm{nn}} \Lambda^{+}{ }_{\mathrm{nn}} \\
\mathrm{C}^{+\mathrm{T}}{ }_{\mathrm{nn}}=\mathrm{R}^{+}{ }_{\mathrm{nn}},\end{array}$ & $\begin{array}{c}\text { эл-ты из } \\
\mathrm{C}^{+}{ }_{2}\end{array}$ & $\mathrm{C}_{2}^{+}$ & $\mathrm{C}_{1}$ \\
\hline OC3 4 & $\begin{array}{c}\left(\Lambda_{\mathrm{nn}}, \mathrm{C}_{\mathrm{nn}}\right)=>\left(\Lambda_{\mathrm{nn}}, \mathrm{C}^{0+}{ }_{\mathrm{nn}}=[\right. \\
\left.\mathrm{C}_{1} \mid \mathrm{C}^{0+}{ }_{2}\right]=>\left(\mathrm{C}^{+}{ }_{\mathrm{nn}}=\right. \\
\left.\left[\mathrm{C}_{1} \mid \mathrm{C}^{+}{ }_{2}\right], \Lambda^{+}{ }_{\mathrm{nn}}\right) .\end{array}$ & $\begin{array}{l}\Lambda_{\mathrm{nn}}^{+}=\operatorname{diag}\left(\lambda_{1}, \ldots\right. \\
\lambda_{\ell}, \lambda^{+} \ell+1, \ldots \\
\left.\lambda_{\mathrm{n}}^{+}\right)\end{array}$ & $\lambda^{+} \ell+1, \ldots, \lambda_{n}^{+}$ & $\begin{array}{c}\mathrm{C}^{+\mathrm{T}}{ }_{n n} \mathrm{C}^{+}{ }_{n n}=\mathrm{C}^{+}{ }_{n n} \mathrm{C} \\
{ }_{+\mathrm{T}}{ }_{\mathrm{nn}}=\mathrm{I}_{\mathrm{nn}}, \mathrm{C}^{+}{ }_{\mathrm{nn}} \Lambda^{+}{ }_{\mathrm{nn}} \\
\mathrm{C}^{+\mathrm{T}}{ }_{\mathrm{nn}}=\mathrm{R}^{+}{ }_{\mathrm{nn}},\end{array}$ & нет & {$\left[\mathrm{C}^{+}{ }_{1} \mid \mathrm{C}^{+}{ }_{2}\right]$} & нет \\
\hline OC3 5 & $\begin{array}{c}\left(\Lambda_{\mathrm{nn}}, \mathrm{C}_{\mathrm{nn}}\right)=>\left(\Lambda_{\mathrm{nn}}, \mathrm{C}^{0}{ }_{\mathrm{nn}}=[\right. \\
\left.\mathrm{C}^{0+}{ }_{1} \mid \mathrm{C}^{0+}{ }_{2}\right]=>\left(\mathrm{C}^{+}{ }_{\mathrm{nn}}=\right. \\
\left.\left[\mathrm{C}_{1} \mid \mathrm{C}^{+}{ }_{2}\right], \Lambda^{+}{ }_{\mathrm{nn}}\right)\end{array}$ & $\begin{array}{l}\Lambda^{+}{ }_{\mathrm{nn}}=\operatorname{diag}\left(\lambda_{1}, \ldots\right. \\
\lambda_{\ell}, \lambda^{+} \ell+1, \ldots \\
\left.\lambda_{\mathrm{n}}^{+}\right)\end{array}$ & $\lambda^{+} \ell+1, \ldots, \lambda_{n}^{+}$ & $\begin{array}{c}\mathrm{C}^{+\mathrm{T}}{ }_{\mathrm{nn}} \mathrm{C}^{+}{ }_{\mathrm{nn}}=\mathrm{C}^{+}{ }_{\mathrm{nn}} \mathrm{C} \\
{ }_{+\mathrm{T}}{ }_{\mathrm{nn}}=\mathrm{I}_{\mathrm{nn}}, \mathrm{C}^{+}{ }_{\mathrm{nn}} \Lambda^{+}{ }_{\mathrm{nn}} \\
\mathrm{C}^{+\mathrm{T}}{ }_{\mathrm{nn}}=\mathrm{R}^{+}{ }_{\mathrm{nn}},\end{array}$ & $\begin{array}{l}\text { Любой эл- } \\
\mathrm{T} \quad \text { из } \\
{\left[\mathrm{C}^{0}{ }_{1} \mid \mathrm{C}^{0}{ }_{2}\right]}\end{array}$ & {$\left[\mathrm{C}^{+}{ }_{1} \mid \mathrm{C}^{+}{ }_{2}\right]$} & нет \\
\hline OC3 6 & $\begin{array}{c}\left(\Lambda_{\mathrm{nn}}, \mathrm{C}_{\mathrm{nn}}\right)=>\left(\Lambda_{\mathrm{nn}}, \mathrm{C}^{0}{ }_{\mathrm{nn}}\right)= \\
>\left(\mathrm{C}^{+}{ }_{\mathrm{nn}}, \Lambda^{+}{ }_{\mathrm{nn}}=\mathrm{I}_{\mathrm{nn}}\right)\end{array}$ & $\begin{array}{l}\Lambda^{+}{ }_{n n}=\operatorname{diag}(1, \ldots 1 \\
)\end{array}$ & $\lambda_{1}, \ldots, \lambda_{n}$ & $\begin{array}{c}\mathrm{C}^{+\mathrm{T}}{ }_{n n} \mathrm{C}^{+}{ }_{n n} \neq \mathrm{I}_{\mathrm{nn}} \\
\mathrm{C}^{+}{ }_{\mathrm{nn}} \mathrm{C}^{+\mathrm{T}}{ }_{\mathrm{nn}}=\mathrm{I}_{\mathrm{nn}}, \mathrm{C}^{+} \\
\mathrm{C}^{+\mathrm{T}}{ }_{\mathrm{nn}}=\mathrm{R}^{+}{ }_{\mathrm{nn}}=\mathrm{I}_{\mathrm{nn}}\end{array}$ & $\begin{array}{c}\text { эл-ты из } \\
\mathrm{C}^{0}{ }_{\mathrm{nn}}\end{array}$ & {$\left[\mathrm{C}^{+} \mid \mathrm{C}^{+}{ }_{2}\right]$} & нет \\
\hline
\end{tabular}

В дополнение к данным, приведенным в таблице 2 заметим, что ОС 33 имеет условие, отсутствующее в ОС 31, в ОС 32. Это условие (дополняет условие из графы 3) формулируется так: полученная полная матрица собственных чисел $\Lambda^{+}{ }_{\mathrm{nnn}}=\operatorname{diag}\left(\lambda_{1}, \ldots, \lambda_{\ell}, \quad \lambda^{+}{ }_{\ell+1}, \ldots, \lambda_{\mathrm{n}}^{+}\right) \quad$ должна иметь заданные значения 6 -параметров $\mathrm{f}_{1}, \mathrm{f}_{2}, \mathrm{f}_{3}, \ell=3, \mathrm{f}_{4}, \mathrm{f}_{5}$, $\mathrm{f}_{6}$ :

$\left.\mathrm{f}_{1}\left(\Lambda_{66}\right)=\lambda_{1}+\lambda_{2}+\ldots+\lambda_{6}\right)=\mathrm{f}_{1}, \quad \mathrm{f}_{2}\left(\Lambda_{66}\right)=\left(\lambda^{2}{ }_{1}++\lambda^{2}{ }_{6}\right)=\mathrm{f}_{2}$, $\mathrm{f}_{3}\left(\Lambda_{66}\right)=\lambda_{1} / \lambda_{6},=\mathrm{f}_{3} \mathrm{f}_{4}\left(\Lambda_{66}\right)=\mathrm{f}_{4}\left(\lambda_{1}+\ldots+\lambda_{3}\right) / 6=\mathrm{f}_{4}$,

$\mathrm{f}_{5}\left(\Lambda_{66}\right)=\lambda_{1} \times \lambda_{2} \times \lambda_{3} \times \ldots \times \lambda_{6}=\mathrm{f}_{5}$,

$\left.\mathrm{f}_{6}\left(\Lambda_{66}\right)=\lambda_{1} / \lambda_{2}+\ldots+\lambda_{5} / \lambda_{6}\right)=\mathrm{f}_{6}$.

Здесь элементы спектра $\lambda_{1}, \ldots, \lambda_{\ell},-$ не помеченные верхним индексом $\left(^{+}\right)$, являются постоянными величинами, а элементы спектра $\lambda^{+} \ell+1, \ldots, \lambda_{\mathrm{n}}^{+}-$помеченные верхним индексом $\left(^{+}\right)$ являются переменными величинами.

\section{Обратная Спектральная Задача ОСЗ 4}

Обратная Спектральная Задача ОC3 4 отличается от ОСЗ5 только тем, что отсутствуют выделенные компоненты собственных векторов из подматриц $\mathrm{C}^{+}{ }_{1}, \mathrm{C}^{+}{ }_{2}$ матрицы $\mathrm{C}^{+}{ }_{\mathrm{nn}}=\left[\mathrm{C}^{+}{ }_{1} \mid \mathrm{C}^{+}{ }_{2}\right]$, соответствующих как постоянным доминирующим, так и изменяющимся недоминирующим собственным числам недоминирующих элементов $\lambda^{+}{ }_{\ell+1}, \ldots, \lambda_{\mathrm{n}}^{+}$спектра. Элементы подматриц $\mathrm{C}^{+}{ }_{1}, \mathrm{C}^{+}{ }_{2}$ матрицы $\mathrm{C}^{+}{ }_{\mathrm{nn}}=\left[\mathrm{C}^{+}{ }_{1} \mid \mathrm{C}^{+}{ }_{2}\right]$ являются изменяемыми величинами, влияющими на формирование значенйе недоминирующих элементов элементов $\lambda^{+}{ }_{\ell+1}, \ldots, \lambda^{+}$спектра $\Lambda^{+}{ }_{n n n}=\operatorname{diag}\left(\lambda_{1}, \ldots, \lambda_{\ell}, \lambda+_{\ell+1}, \ldots, \lambda_{n}^{+}\right)$.

Излагаемая ниже ОС35 является задачей моделирования матрицы $\mathrm{C}_{\mathrm{nn}}$ собственных векторов корреляционной матрицы, согласованной с матрицей собственных чисел $\Lambda_{n n}=\operatorname{diag}\left(\lambda_{1}, \ldots, \lambda_{n}\right)$ корреляционной матрицы, при заданных значениях доминирующих $\lambda_{1}, \ldots, \lambda_{\ell}$ и заданных значениях компонент соответствующих им собственных векторов $\mathrm{C}_{1}=\left[\mathbf{c}_{1}\left|\mathbf{c}_{2}\right| \mathbf{c}_{\ell}\right] . \mathrm{B}$ ОС 35 моделируются недоминирующим собственным числа и соответствующая подматрица $\mathrm{C}^{+}{ }_{2}$ матрицы $\mathrm{C}^{+}{ }_{\mathrm{nn}}=\left[\mathrm{C}^{+}{ }_{1} \mid \mathrm{C}^{+}{ }_{2}\right]$ собственных векторов. Эта задача является весьма актуальной, имеет много приложений. Так как ОС34 является частным случаем ОС 35 , то здесь не приводим описание ее решения.

Если значения элементов $\lambda_{\ell+1}^{+}, \ldots, \lambda_{n}^{+}$(столбец 4, Таблица 1) считать отсутствующими, то задача OC34 будет являться Задачей Оценки Отсутствующих Недоминирующих Значений Собственных Чисел (задача ООНЗСЧ, ). 


\begin{tabular}{|c|c|c|c|c|c|c|}
\hline \multirow{4}{*}{ Impact Factor: } & ISRA (India) & $=3.117$ & SIS (USA) & $=0.912$ & ICV (Poland) & $=6.630$ \\
\hline & ISI (Dubai, UAE & $=0.829$ & РИНЦ (Russia) & $=0.156$ & PIF (India) & $=1.940$ \\
\hline & GIF (Australia) & $=0.564$ & ESJI (KZ) & $=8.716$ & IBI (India) & $=4.260$ \\
\hline & JIF & $=1.500$ & SJIF (Morocco) & $=5.667$ & OAJI (USA) & $=0.350$ \\
\hline
\end{tabular}

\section{Обратная Спектральная Задача ОСЗ 5}

Обратная Спектральная Задача ОСЗ5 является обобщением ОС 34 . Отличие ОС 35 от ОС 34 состоит в том, что в ОС34 нет ни одного выделенного элемента в начальной матрице $\mathrm{C}_{\text {nn }}^{0}$ собственных векторов, а в ОС 35 имеется хотя бы один выделенный элемент $\mathrm{c}_{\mathrm{ij}}$ с заданным значением фиксированным пользователем или случайным (из интервала $[-1,+1])$. Выделенное значение должно принадлежать одному из 5-ти интервалов шкалы Чэддока Chaddock scale). По этой шкале количественная мера тесноты связи между i-ой упеременной и $\mathrm{j}$-ой $\mathrm{z}$-переменной измеряется величиной коэффициента корреляции $\mathrm{c}_{\mathrm{ij}}=\operatorname{corr}\left(\mathrm{y}_{\mathrm{i}}, \mathrm{z}_{\mathrm{j}}\right)$ : абсолютное значение коэффициента корреляции, принадлежащее интервалу от 0 до 0.3 - качественно интерпретируется как «очень слабая», интервалу от 0.3 до 0.5 -«слабая, умеренная», интервалу от 0.5 до 0.7-«заметная» (moderate positive), интервалу от 0.7 до 0.9-«высокая», интервалу от 0.9 до 1-«очень высокая». Значения коэффициентов корреляции $\mathrm{c}_{\mathrm{ij}}=\operatorname{corr}\left(\mathrm{y}_{\mathrm{i}}, \mathrm{z}_{\mathrm{j}}\right)$ объединены в несимметрическую матрицу $\mathrm{C}_{\mathrm{nn}}=\left[\mathrm{c}_{\mathrm{ij}}=\operatorname{corr}\left(\mathrm{y}_{\mathrm{i}}, \mathrm{Z}_{\mathrm{j}}\right)\right], \mathrm{i}=1, \ldots, \mathrm{n} ; \mathrm{j}=1, \ldots, \mathrm{n}$.

Одно выделенное значение, отличающееся от предыдущего значения, меняет всю матрицу $\mathrm{C}^{+}{ }_{\mathrm{nn}}-$ одно из решений ОС 35 . Для каждого случайного значения выделенного элемента $\left.\mathrm{c}^{(\ell)}\right)_{\mathrm{ij}}$, с номером $\ell=1, \ldots, \propto, \quad$ принадлежащему интервалу $[-1,+1]$, существует решение ОС 35 -матрица $\mathrm{C}^{+(\ell)}{ }_{n n}$ с номером $\ell=1, \ldots, \propto$, удовлетворяющее условиям $\mathrm{C}^{+(\ell) \mathrm{T}}{ }_{\mathrm{nn}} \mathrm{C}^{+(\ell)}{ }_{\mathrm{nn}}=$ $\mathrm{C}^{+(\ell)}{ }_{\mathrm{nn}} \mathrm{C}^{+(\ell) \mathrm{T}}{ }_{n n}=\mathrm{I}_{\mathrm{nn}}, \mathrm{C}^{+(\ell)}{ }_{n n} \Lambda^{+}{ }_{\mathrm{nn}} \mathrm{C}^{+(\ell) \mathrm{T}}{ }_{\mathrm{nn}}=\mathrm{R}^{+(\ell)}{ }_{\mathrm{nn}}$. Обратная Спектральная Задача ОС35 имеет решение - пару матриц $\left(\mathrm{C}^{+(\ell)}{ }_{\mathrm{nn}}, \Lambda^{+(\ell)}{ }_{\mathrm{nn}}\right)$. При этом одна матрица $\Lambda^{+(\ell)}{ }_{\mathrm{nn}}=\operatorname{diag}\left(\lambda_{1}, \ldots \lambda_{\ell}, \lambda^{+} \ell+1, \ldots, \lambda_{\mathrm{n}}^{+}\right) \quad$ из пары матриц $\left(\mathrm{C}^{+(\ell)}{ }_{\mathrm{nn}}, \Lambda^{+(\ell)}{ }_{\mathrm{nn}}\right)$ имеет одни и те же заданные значения 6 -параметров $\mathrm{f}_{1}, \mathrm{f}_{2}, \mathrm{f}_{3}, \ell=3, \mathrm{f}_{4}, \mathrm{f}_{5}, \mathrm{f}_{6}$ :

$\left.\mathrm{f}_{1}\left(\Lambda^{+}{ }_{\mathrm{nn}}\right)=\lambda_{1}+\lambda_{2}+\ldots+\lambda_{\mathrm{n}}^{+}\right)=\mathrm{f}_{1}, \quad \mathrm{f}_{2}\left(\Lambda^{+}{ }_{\mathrm{nn}}\right)=\left(\lambda^{2}{ }_{1}++\lambda^{+2}{ }_{\mathrm{n}}\right)=\mathrm{f}_{2}$, $\mathrm{f}_{3}\left(\Lambda^{+}{ }_{\mathrm{nn}}\right)=\lambda_{1} / \lambda^{+}{ }_{\mathrm{n}}=\mathrm{f}_{3}, \quad \mathrm{f}_{4}\left(\Lambda^{+}{ }_{\mathrm{nn}}\right)=\mathrm{f}_{4}\left(\lambda_{1}+\ldots+\lambda_{\ell}\right) / \mathrm{n}=\mathrm{f}_{4}$, $\mathrm{f}_{5}\left(\Lambda_{\mathrm{nn}}\right)=\lambda_{1} \times \lambda_{2} \times \ldots \lambda^{+} \ell+\lambda_{1} \times \lambda^{+}{ }_{n}=\mathrm{f}_{5}$,

$\left.\mathrm{f}_{\mathrm{n}}\left(\Lambda^{+}{ }_{\mathrm{nn}}\right)=\lambda_{1} / \lambda_{2}+\ldots+\lambda_{\mathrm{n}-1}^{+} / \lambda_{\mathrm{n}}^{+}\right)=\mathrm{f}_{\mathrm{n}}$.

ОС35 является альтернативной моделью к модели ОС31 [3]. Отличие ОС 35 от ОС31 состоит в постоянстве доминирующих элементов $\lambda_{1}, \ldots \lambda_{\ell}$, в возможности фиксировать заданные значения выделенным элементам матрицы $\mathrm{C}^{+(\ell)}{ }_{n n}$. свойство матрицы $\Lambda^{+(\ell)}{ }_{n n}=\operatorname{diag}\left(\lambda_{1}, \ldots \lambda_{\ell}, \quad \lambda_{\ell+1}^{+}, \ldots, \lambda_{\mathrm{n}}^{+}\right) \quad$ иметь заданные значения $6 \mathrm{f}$-параметров $\mathrm{f}_{1}, \mathrm{f}_{2}, \mathrm{f}_{3}, \mathrm{f}_{4}, \mathrm{f}_{5}, \mathrm{f}_{6}$ присуще ОС 31 , если входной объект $\Lambda_{\mathrm{nn}}$ ОС 31 : $\Lambda_{\mathrm{nn}}=>\left(\mathrm{C}^{(\ell)}{ }_{\mathrm{nn}}, \mathrm{R}^{(\ell)}{ }_{\mathrm{nn}}\right), \ell=1, \ldots, \mathrm{k}_{\ell}<\infty$, был смоделирован как спектр $\Lambda_{\mathrm{nn}}=\operatorname{diag}\left(\lambda_{1}, \ldots, \lambda_{\mathrm{n}}\right)$, полученный в результате решения Оптимизационной задачи с линеаризованными уравнениями $\mathrm{f}$-параметров. Этот спектр заданные значения $\mathrm{f}$-параметров и назван « $\left(\mathrm{f}_{1}, \mathrm{f}_{2}, \mathrm{f}_{3}, \mathrm{f}_{4}, \mathrm{f}_{5}, \mathrm{f}_{6}\right)$-спектр». Если в ОС3 1 ее входной объект $\Lambda_{\mathrm{nn}}=\operatorname{diag}\left(\lambda_{1}, \ldots, \lambda_{\mathrm{n}}\right) \quad$ является $\left(\mathrm{f}_{1}, \mathrm{f}_{2}, \mathrm{f}_{3}, \mathrm{f}_{4}, \mathrm{f}_{5}, \mathrm{f}_{6}\right)$-спектром, то он может быть входным объектом ОС35. Если же вычислить значения $\mathrm{f}-$ параметров входного параметра ОС 31 и, если эти значения пользователь признает пригодными (подходящими) для его исследований (что маловероятно), то спектр $\Lambda_{\mathrm{nn}}=\operatorname{diag}\left(\lambda_{1}, \ldots, \lambda_{\mathrm{n}}\right)$ может быть взят в качестве входного объекта ОС 35 . Самым приоритетным случаем является вариант, когда $\Lambda_{\mathrm{nn}}=\operatorname{diag}\left(\lambda_{1}, \ldots, \lambda_{\mathrm{n}}\right) \quad$ является спектром корреляционной матрицы $\mathrm{R}_{\mathrm{nn}}$ реальной многомерной выборки. Матрица $\mathrm{R}_{\mathrm{nn}}$ подчиняется равенствам: $\quad \mathrm{R}_{\mathrm{nn}}=\mathrm{R}^{\mathrm{T}}{ }_{\mathrm{nn}}, \quad \mathrm{R}_{\mathrm{nn}} \mathrm{C}_{\mathrm{nn}}=\mathrm{C}_{\mathrm{nn}} \Lambda_{\mathrm{nn}}$, $\mathrm{C}^{\mathrm{T}}{ }_{\mathrm{nn}} \mathrm{C}_{\mathrm{nn}}=\mathrm{C}_{\mathrm{nn}} \mathrm{C}^{\mathrm{T}}{ }_{n n}=\mathrm{I}_{\mathrm{nn}}, \quad \operatorname{diag}\left(\mathrm{R}_{\mathrm{nn}}\right)=(1, \ldots, 1)$, $\operatorname{tr}\left(R_{\mathrm{nn}}\right)=1+\ldots+1=\operatorname{tr}\left(\Lambda_{\mathrm{nn}}\right)=\lambda_{1}+\ldots+\lambda_{\mathrm{n}}=\mathrm{n}, \quad \lambda_{1} \geq \ldots \quad \geq \lambda_{\mathrm{n}} \geq 0$ $[1,2]$.

\section{Обратная Спектральная Задача №6 OC3 6}

Рассмотрим случай $\Lambda_{\mathrm{nn}}=\mathrm{I}_{\mathrm{nn}}, \lambda_{1}=\ldots=\lambda_{\mathrm{n}}=1$. Равенство собственных чисел другу означает равенство дисперсий $\mathrm{y}$-переменных одной константе, равенство количества информации, содержащейся в каждом из них, друг другу. В других спектральных задачах предполагалось наличие доминирующих элементов. Такое свойство спектра присуще для спектра корреляционной матрицы $\mathrm{R}_{\mathrm{nn}}$ реальной многомерной выборки.

Теперь мы рассматриваем гипотетические случаи существования матрицы собственных векторов $\mathrm{C}_{\mathrm{nn}}$, обладающих достаточно сильно выраженными свойствами. Например, «вес» при zпеременной, вычисленный по реальной многомерной выборке, равен 0.5191. Это значение принадлежит одному из 5-ти интервалов шкалы Чэддока - интервалу от 0.5 до 0.7-«заметная» (moderate positive). Необходимо «передвинуть» это значение к правому краю интервала - к точке 0.7000 , например, заменить число 0.5191 на число 0.6500 . Этим мы усиливаем «вес». Некоторые «веса» необходимо ослабить. Такие действия уместны при когнитивном восприятии сил взаимосвязей между iой у-переменной и j-ой $\mathrm{z}$-переменной, измеряемой величиной коэффициента корреляции $\mathrm{c}_{\mathrm{ij}}=\operatorname{corr}\left(\mathrm{y}_{\mathrm{i}}, \mathrm{z}_{\mathrm{j}}\right)=0.6500, \quad$ принадлежащей к правой границе интервалу от 0.5 до 0.7 - качественно интерпретируется как «заметная». Более «заметная» связь одних пар (y, z) переменных по сравнению с «заметной» связью другой пары $(\mathrm{y}, \mathrm{z})$ переменных адекватно отражает то, что наблюдается на практике. Такие изменения значений некоторых выделенных компонент собственных векторов $\mathbf{c}_{\mathbf{j}}=\left(\mathrm{c}_{1 \mathrm{j}}, \mathrm{c}_{2 \mathrm{j}} \ldots \mathrm{c}_{\mathrm{nj}}\right)^{\mathrm{T}}$, $\mathrm{j} \in\{1, \ldots, \mathrm{n}\}$, позволяет при правильном выборе их значений переходить от одного «реальной» матрицы к другой «реальной» матрице $\mathrm{C}^{+}{ }_{\mathrm{nn}}$. Они соответствуют разным реальным ситуациям. Пока рассмотрим гипотетическую когнитивную ситуацию отсутствия парных связей между zпеременными, равенства единице дисперсий всех, n 


\begin{tabular}{|c|c|c|c|c|c|c|}
\hline \multirow{4}{*}{ Impact Factor: } & ISRA (India) & $=3.117$ & SIS (USA) & $=0.912$ & ICV (Poland) & $=6.630$ \\
\hline & ISI (Dubai, UAE & $=\mathbf{0 . 8 2 9}$ & РИНЦ (Russia) & $=\mathbf{0 . 1 5 6}$ & PIF (India) & $=1.940$ \\
\hline & GIF (Australia) & $=0.564$ & ESJI (KZ) & $=8.716$ & IBI (India) & $=4.260$ \\
\hline & JIF & $=1.500$ & SJIF (Morocco) & $=5.667$ & OAJI (USA) & $=0.350$ \\
\hline
\end{tabular}

z-переменных и дисперсий всех, n y-переменных. Требуется моделировать другую, отличную от данной «реальной» матрицы $\mathrm{C}_{\mathrm{nn}}$, «реальную» матрицу $\mathrm{C}^{+}{ }_{n n}$ с заданными значениями выделенных компонент в собственных векторах $\mathbf{c}_{\mathrm{j}}=\left(\mathrm{c}_{1 \mathrm{j}}, \mathrm{c}_{2 \mathrm{j}} \ldots \mathrm{c}_{\mathrm{nj}}\right)^{\mathrm{T}}$, $\mathrm{j} \in\{1, \ldots, \mathrm{n}\}$. В новой Обратной Спектральной Задаче ОСЗ 6 требуется при $\mathrm{R}^{+}{ }_{\mathrm{nn}}=\mathrm{I}_{\mathrm{nn}}, \Lambda^{+}{ }_{\mathrm{nn}}=\mathrm{I}_{\mathrm{nn}}$ моделировать матрицу $\mathrm{C}^{+}{ }_{n n}$, удовлетворяющую равенству I ${ }_{\mathrm{nn}} \mathrm{C}^{+}{ }_{\mathrm{nn}}=\mathrm{C}^{+}{ }_{\mathrm{nn}} \mathrm{I}_{\mathrm{nn}}$ (вместо равенства $\mathrm{R}^{+} \mathrm{C}^{+}{ }_{\mathrm{nn}}=\mathrm{C}^{+}{ }_{\mathrm{nn}} \Lambda^{+}{ }_{\mathrm{nn}}$ ). Начальной матрицей является «реальная» матрица $\mathrm{C}^{0}{ }_{n}$, в ней выделенные элементы имеют заданные значения, о которых мы выше привели когнитивное обоснование.

Назначенные новые значения нарушают присущие матрице собственных векторов равенства: $\mathrm{C}^{+\mathrm{T}}{ }_{n n} \mathrm{C}^{+}{ }_{n n}=\mathrm{I}_{\mathrm{nn}}, \mathrm{C}^{+}{ }_{\mathrm{nn}} \mathrm{C}^{+\mathrm{T}}{ }_{\mathrm{nn}}=\mathrm{I}_{\mathrm{nn}}$. Так как в матрице $\mathrm{C}_{\mathrm{nn}}$ собственные векторы $\mathbf{c}_{\mathbf{j}}=\left(\mathrm{c}_{1 \mathrm{j}}, \mathrm{c}_{2 \mathrm{j}} \ldots \mathrm{c}_{\mathrm{nj}}\right)^{\mathrm{T}}$, взаимно ортогональны $\mathbf{c}_{\mathrm{j}}^{\mathrm{T}} \mathbf{c}_{\mathrm{j}}=0$, при $\mathrm{i} \neq \mathrm{j}$, и имеют единичную дину $\mathbf{c}_{\mathrm{j}}^{\mathrm{T}} \mathbf{c}_{\mathrm{j}}=1$, то в модельной матрице $\mathrm{C}^{+}{ }_{n n}$ не будем требовать единичной дины для всех векторов $\mathbf{c}_{\mathbf{j}}{ }^{+}=\left(\mathrm{c}^{+}{ }_{1 \mathrm{j}}, \mathrm{c}^{+}{ }_{2 \mathrm{j}} \ldots \mathrm{c}^{+}{ }_{\mathrm{nj}}\right)^{\mathrm{T}} \mathbf{c}_{\mathrm{j}}{ }^{+\mathrm{T}} \mathbf{c}_{\mathrm{j}}{ }^{+} \neq 1, \mathrm{j} \in\{1, \ldots, \mathrm{n}\} . \quad$ Условие ортогональны $\mathbf{c}_{\mathrm{j}}^{\mathrm{T}} \mathbf{c}_{\mathrm{j}}=0$, при $\mathrm{i} \neq \mathrm{j}$, оставим в силе. Тогда в матричном виде ограничения на модельную матрицу псевдособственных векторов примут вид: $\mathrm{C}^{+\mathrm{T}}{ }_{\mathrm{nn}} \mathrm{C}^{+}{ }_{\mathrm{nn}} \neq \mathrm{I}_{\mathrm{nn}}, \mathrm{C}^{+}{ }_{\mathrm{nn}} \mathrm{C}^{+\mathrm{T}}{ }_{\mathrm{nn}}=\mathrm{I}_{\mathrm{nn}}$. Тогда будут выполняться условия для нулевых значений коэффициентов корреляции z-переменных $\mathbf{c}_{\mathrm{i}}^{+} \Lambda^{+}{ }_{\mathrm{nn}} \mathbf{c}_{\mathrm{j}}{ }^{+\mathrm{T}}=\mathrm{r}^{+}{ }_{\mathrm{ij}}=0$. Так как $\mathrm{C}^{+}{ }_{\mathrm{nn}} \mathrm{C}^{+\mathrm{T}}{ }_{\mathrm{nn}}=\mathrm{I}_{\mathrm{nn}}, \Lambda^{+}{ }_{\mathrm{nn}}=\mathrm{I}_{\mathrm{nn}}, \mathrm{Y}_{\mathrm{mn}}=\mathrm{U}_{\mathrm{mn}} \Lambda^{(1 / 2)+}{ }_{\mathrm{nn}}=\mathrm{U}_{\mathrm{mn}} \mathrm{I}^{1 / 2}{ }_{\mathrm{nn}}=\mathrm{U}_{\mathrm{mn}}$, $\mathrm{Z}_{\mathrm{mn}}=\mathrm{Y}_{\mathrm{mn}} \mathrm{C}^{+\mathrm{T}}{ }_{\mathrm{nn}}=\mathrm{U}_{\mathrm{mn}} \mathrm{C}^{+\mathrm{T}}{ }_{\mathrm{nn}}$, то имеем равенство $\mathrm{R}^{+}{ }_{\mathrm{nn}}=(1 / \mathrm{m}) \mathrm{Z}_{\mathrm{mn}}{ }^{\mathrm{T}} \mathrm{Z}_{\mathrm{mn}}=(1 / \mathrm{m})\left[\mathrm{U}_{\mathrm{mn}} \mathrm{C}^{+\mathrm{T}}{ }_{\mathrm{nn}}\right]^{\mathrm{T}}\left[\mathrm{U}_{\mathrm{mn}} \mathrm{C}^{+\mathrm{T}}{ }_{\mathrm{nn}}\right]=(1 / \mathrm{m})$ $\mathrm{C}^{+}{ }_{n n}\left(\mathrm{U}_{\mathrm{mn}}{ }^{\mathrm{T}}\left[\mathrm{U}_{\mathrm{mn}}\right) \mathrm{C}^{+\mathrm{T}}{ }_{\mathrm{nn}}\right]=\mathrm{C}^{+}{ }_{\mathrm{nn}} \mathrm{C}^{+\mathrm{T}}{ }_{\mathrm{nn}}=\mathrm{I}_{\mathrm{nn}}$. Равенство $\mathrm{R}^{+}{ }_{\mathrm{nn}}=$ $\mathrm{I}_{\mathrm{nn}}$ преобразует основное равенство ПСЗ $\mathrm{R}^{+}{ }_{n n} \mathrm{C}^{+}{ }_{n n}=\mathrm{C}^{+}{ }_{n n} \Lambda^{+}{ }_{n n} \quad$ в $\quad$ равенство $\mathrm{C}^{+}{ }_{\mathrm{nn}}=\mathrm{C}^{+}{ }_{\mathrm{nn}}$. Геометрическая интерпретация равенств $\mathrm{C}^{+\mathrm{T}}{ }_{\mathrm{nn}} \mathrm{C}^{+}{ }_{\mathrm{nn}} \neq \mathrm{I}_{\mathrm{nn}}, \mathrm{C}^{+}{ }_{\mathrm{nn}} \mathrm{C}^{+\mathrm{T}}{ }_{\mathrm{nn}}=\mathrm{I}_{\mathrm{nn}}$, следующая: облако точек $\left(\mathrm{z}_{\mathrm{i} 1}, \ldots, \mathrm{z}_{\mathrm{in}}\right), \mathrm{i}=1, \ldots, \mathrm{m}, \quad$ сосредоточено в $\mathrm{n}$-мерном шаре с радиусом, длина которого пропорциональна 1. Они получены путем умножения векторов $\left(\mathrm{y}_{\mathrm{i} 1}, \ldots, \mathrm{y}_{\mathrm{in}}\right)$ справа на матрицу $\mathrm{C}^{+\mathrm{T}}{ }_{\mathrm{nn}}$. Матрица $\mathrm{Y}_{\mathrm{mn}}=\mathrm{U}_{\mathrm{mn}}$ моделируется отдельно и независимо от матриц $\mathrm{C}^{+}{ }_{n n}$ и $\Lambda^{+}{ }_{n n}$. Точки $\left(\mathrm{y}_{\mathrm{i} 1}, \ldots, \mathrm{y}_{\mathrm{in}}\right), \mathrm{i}=1, \ldots, \mathrm{m}$, по построению находятся в шаре с радиусом, длина которого пропорциональна 1.

\section{Визуализация облака точек внутри n- мерного шара}

Для пар (i,j) изобразим разные облака точек $\left\{\left(\mathrm{z}_{\mathrm{ki}}, \mathrm{z}_{\mathrm{kj}}\right)\right\}, \mathrm{i} \neq \mathrm{j}, \quad \mathrm{k}=1, . ., \mathrm{m},\left\{\left(\mathrm{y}_{\mathrm{ki}}, \mathrm{y}_{\mathrm{kj}}\right)\right\} . \quad$ Аналогично построим точки $\left\{\left(\mathrm{z}_{\mathrm{ki}}, \mathrm{Z}_{\mathrm{kj}}\right)\right\}, \mathrm{i} \neq \mathrm{j}, \mathrm{k}=1, . ., \mathrm{m}$, и для пар $(\mathrm{i}, \mathrm{j})$ изобразим другие облака точек $\left\{\left(\mathrm{z}_{\mathrm{ki}}, \mathrm{z}_{\mathrm{kj}}\right)\right\}, \mathrm{i} \neq \mathrm{j}$, $\mathrm{k}=1, . ., \mathrm{m}$. Изобразим на 2-мерной точечной диаграмме возможные точки ( $\left.\mathrm{y}_{\mathrm{ki}}, \mathrm{y}_{\mathrm{kj}}\right), \mathrm{i} \neq \mathrm{j}, \mathrm{k}=1, . ., \mathrm{m}$. Пар координат из 6 координат будет 15. Объединение 2-мерных плоскостей в 6-мерное евклидово пространство $\mathrm{E}^{6}$ с взаимно перпендикулярными осями координат даст 6мерный шар. Для иллюстрации двух облаков точек $\left\{\left(\mathrm{z}_{\mathrm{ki}}, \mathrm{z}_{\mathrm{kj}}\right)\right\}, \quad \mathrm{i} \neq \mathrm{j}, \quad \mathrm{k}=1, . ., \mathrm{m}, \quad\left\{\left(\mathrm{y}_{\mathrm{ki}}, \mathrm{y}_{\mathrm{kj}}\right)\right\}, \quad \mathrm{i} \neq \mathrm{j}, \quad \mathrm{k}=1, . ., \mathrm{m}$, нарисованы 30 точечных диаграмм. Ниже приведена одна пара точечных диаграмм для двух облаков точек $\left\{\left(\mathrm{z}_{\mathrm{k} 1}, \mathrm{z}_{\mathrm{k} 2}\right)\right\}, \mathrm{k}=1, . .20,\left\{\left(\mathrm{y}_{\mathrm{k} 1}, \mathrm{y}_{\mathrm{k} 2}\right)\right\}, \mathrm{k}=1, . .20$. Для каждой пары $(\mathrm{i}, \mathrm{j})$ визуально сравним 2 облака и убедимся в отсутствии аппроксимирующего эллипса для облака точек. Убедимся: все точки сосредоточены в n-мерном шаре неединичного радиуса, а преобразованные точки $\left(\mathrm{y}_{\mathrm{i} 1}, \ldots, \mathrm{y}_{\mathrm{in}}\right)$, $\mathrm{i}=1, \ldots, \mathrm{m}$, также находятся в шаре. Рисунки 2-х облаков для $(1,2)$-пар у-переменных $\left(\mathrm{y}_{\mathrm{i} 1}, \mathrm{y}_{\mathrm{i} 2}\right), \mathrm{i}=1, \ldots$, 20 (Рисунок 1), и z-переменных $\left\{\left(\mathrm{z}_{\mathrm{i} 1}, \mathrm{z}_{\mathrm{i} 2}\right)\right\}, \mathrm{i}=1, \ldots, 20$ (Рисунок 2). Изображения этих облаков точек вписываются в круги с радиусами 3 и 2.5. в отличие от изображений облаков точек на Рисунках 3 и 4 [6,Рисунки 1 и 2]. Изображения облаков точек на Рисунках 3 и 4 вписываются в эллипсы с длинами полуосей, равными $\lambda_{1}=1+\mathrm{r}_{12}, \lambda_{2}=1-\mathrm{r}_{12}$. Рисунки 3 и 4 иллюстрируют случай наличия заметной

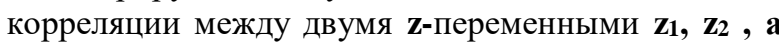
Рисунки 1 и 2 иллюстрируют случай полного отсутствия корреляций между двумя zпеременными $z_{1}, z_{2}$. Объединяя 2-мерные плоскости в 6-мерное евклидово пространство $\mathrm{E}^{6}$ с взаимно перпендикулярными осями координат имеем 6мерный шар. Два шара содержат внутри себя 2 облака из 20 точек $\left\{\left(\mathrm{z}_{\mathrm{k} 1}, \ldots, \mathrm{z}_{\mathrm{k} 6}\right)\right\},\left\{\left(\mathrm{y}_{\mathrm{k} 1}, \ldots, \mathrm{y}_{\mathrm{k} 6}\right)\right\}$, $\mathrm{k}=1, . ., 20$. объединив точки из нескольких $\mathrm{I}_{\mathrm{nn}}$ выборок $\mathrm{Z}^{(\mathrm{t})} 20,6, \mathrm{t}=1, \ldots, \mathrm{k}_{\mathrm{t}}<\propto$, имеем объединенные $\mathrm{I}_{\mathrm{nn}}$-выборки $\mathrm{Z}_{\mathrm{M} 6}$ объемов $\mathrm{M}=40,60, \ldots, 20 \mathrm{k}_{\mathrm{t}}<\propto$, составленные из 2-х, 3-х или $\mathrm{k}_{\mathrm{t}} \mathrm{I}_{\mathrm{nn}}-$-выборок $\mathrm{Z}^{(\mathrm{t})} 20,6$. Объединенная выборка $Z_{40,6}$ состоит из присоединения выборки $Z^{(1)}{ }_{20,6}$ и выборки $Z^{(2)}{ }_{20,6}$, выборка $Z_{60,6}$ состоит из объединения 3-х выборок: $Z^{(1)}{ }_{20,6}, Z^{(2)}{ }_{20,6}$ и $Z^{(3)}{ }_{20,6}$. $\mathrm{I}_{\text {nn }}$-выборки $Z^{(\mathrm{t})}{ }_{20,6}$ с любыми номерами $\mathrm{t}$ могут быть объединены в $\mathrm{I}_{\mathrm{nn}}$-выборку $\mathrm{Z}_{\mathrm{M} 6}$ объема $\mathrm{M}=40,60, \ldots, 20 \mathrm{k}_{\mathrm{t}}<\propto$, строки в них могут быть переставлены местами - корреляционная матрица не изменится: (1/M) $\mathrm{Z}^{\mathrm{T}}{ }_{\mathrm{M} 6} \mathrm{Z}_{\mathrm{M} 6}=\mathrm{I}_{\mathrm{nn}}$. Здесь $\mathrm{I}_{\mathrm{nn}}$ выборка обозначает $\Lambda_{\mathrm{nn}}-$ выборку при $\Lambda_{\mathrm{nn}}=\mathrm{I}_{\mathrm{nn}}$.

В результате решения ОС 36 «фигура из точек» из Рисунка 1 преобразуется в «фигуру из точек» на Рисунка 2. Такой случай рассматривается впервые, задача приписывания когнитивных независимых смыслов некоррелированным z-переменным рассматривается впервые. 


\begin{tabular}{|c|c|c|c|c|c|c|}
\hline \multirow{4}{*}{ Impact Factor: } & ISRA (India) & $=3.117$ & SIS (USA) & $=0.912$ & ICV (Poland) & $=6.630$ \\
\hline & ISI (Dubai, UAE & $=0.829$ & РИНЦ (Russia) & $=\mathbf{0 . 1 5 6}$ & PIF (India) & $=1.940$ \\
\hline & GIF (Australia) & $=0.564$ & ESJI (KZ) & $=8.716$ & IBI (India) & $=4.260$ \\
\hline & JIF & $=1.500$ & SJIF (Morocco) & $=5.667$ & OAJI (USA) & $=0.350$ \\
\hline
\end{tabular}

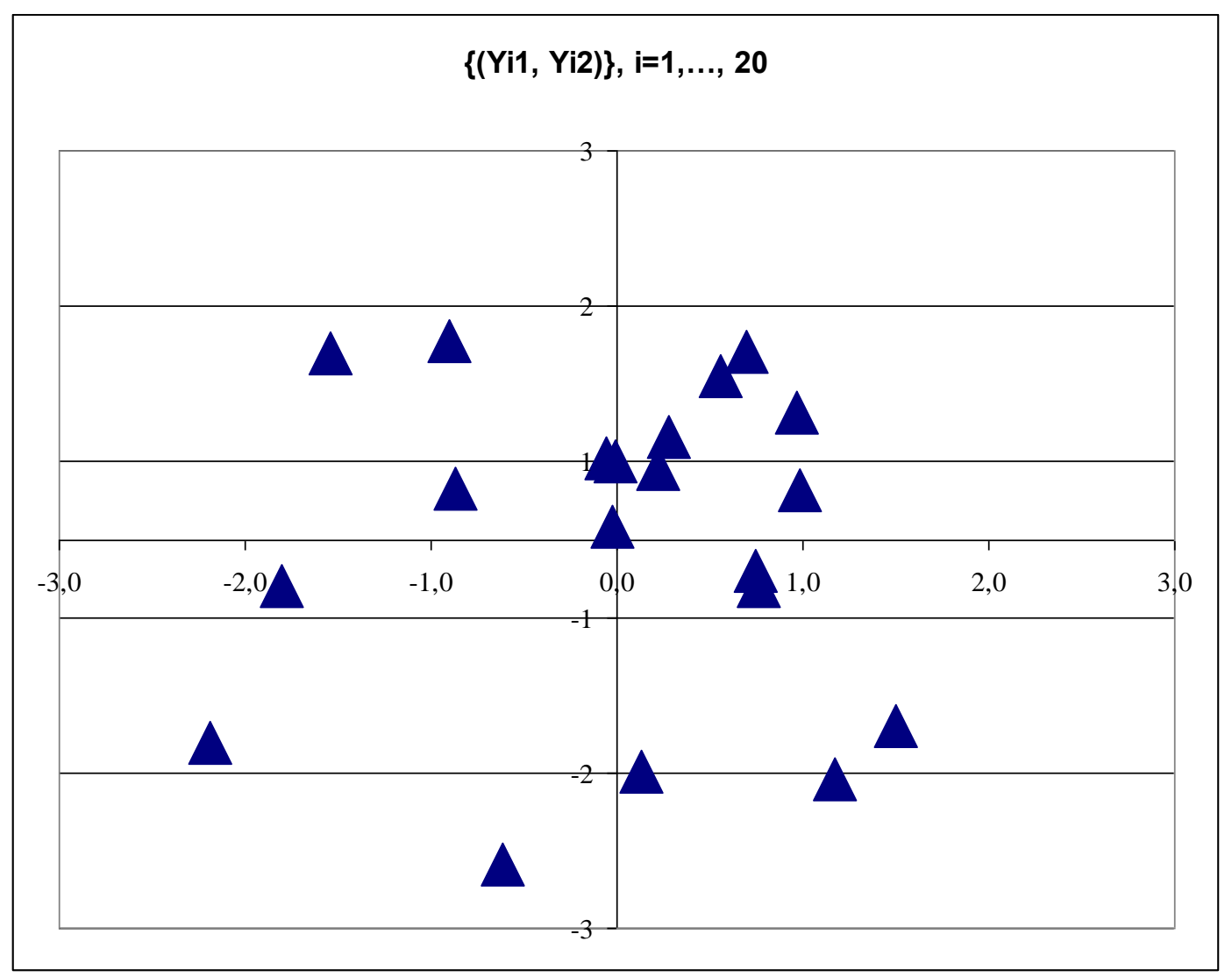

Рисунок 1. Облако точек с координатами

$\left(\mathbf{y}_{\mathbf{i} 1}, \mathbf{y}_{\mathrm{i} 2}\right), \mathrm{i}=\mathbf{1}, \ldots, \mathbf{2 0}, \mathbf{\Lambda}_{\mathbf{6 6}}=\operatorname{diag}(1, \ldots, 1)$ 


\begin{tabular}{|c|c|c|c|c|c|c|}
\hline \multirow{4}{*}{ Impact Factor: } & ISRA (India) & $=3.117$ & SIS (USA) & $=0.912$ & ICV (Poland) & $=6.630$ \\
\hline & ISI (Dubai, UAE & $=0.829$ & РИНЦ (Russia) & $=0.156$ & PIF (India) & $=1.940$ \\
\hline & GIF (Australia) & $=0.564$ & ESJI (KZ) & $=8.716$ & IBI (India) & $=4.260$ \\
\hline & JIF & $=1.500$ & SJIF (Morocco) & $=5.667$ & OAJI (USA) & $=0.350$ \\
\hline
\end{tabular}

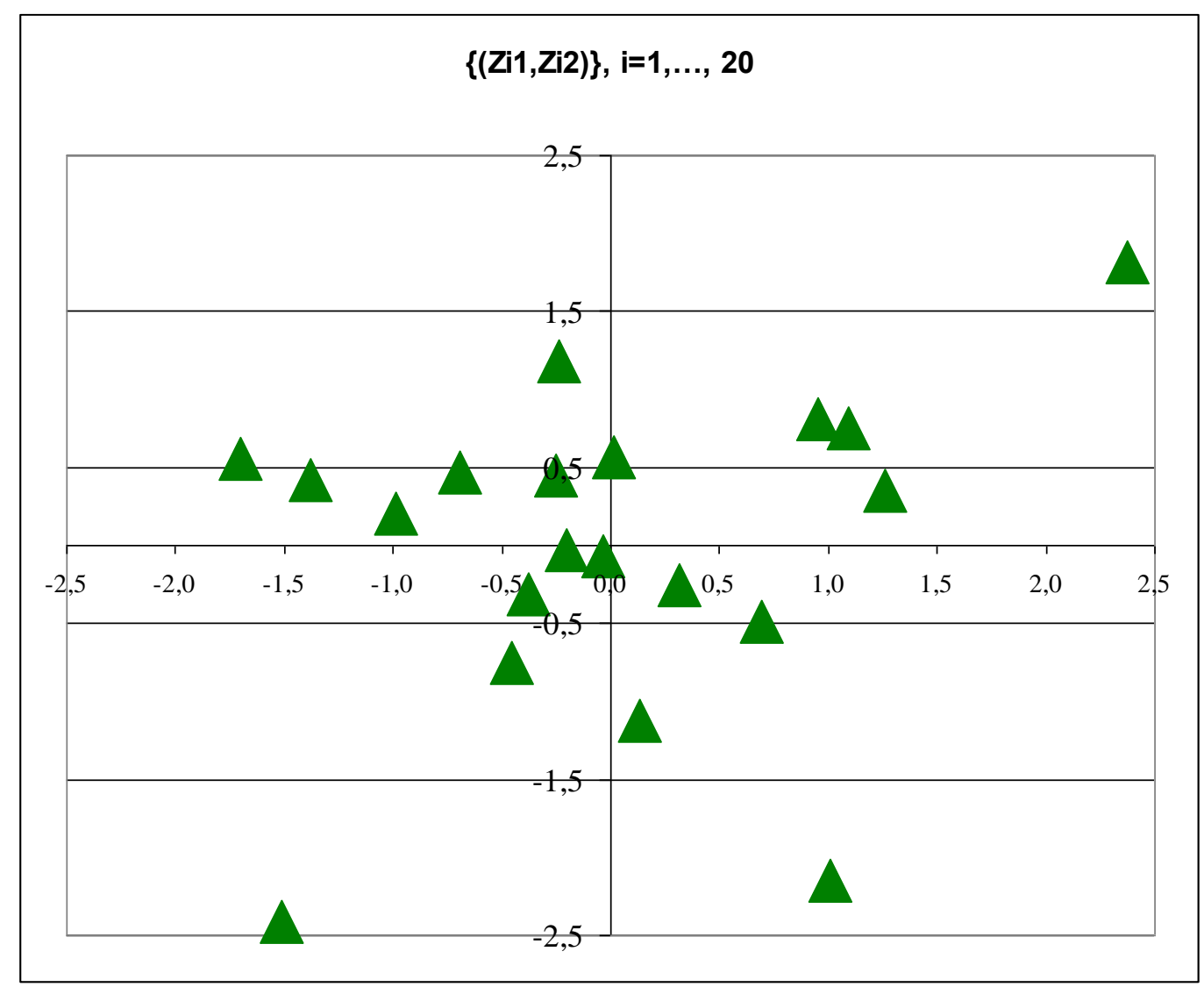

Рисунок 2. Облако точек с координатами

$\left\{\left(\mathbf{z}_{\mathbf{i} 1}, \mathbf{z}_{\mathbf{i} 2}\right)\right\}, \mathrm{i}=\mathbf{1}, \ldots, \mathbf{2 0}, \Lambda_{66}=\operatorname{diag}(1, \ldots, 1)$

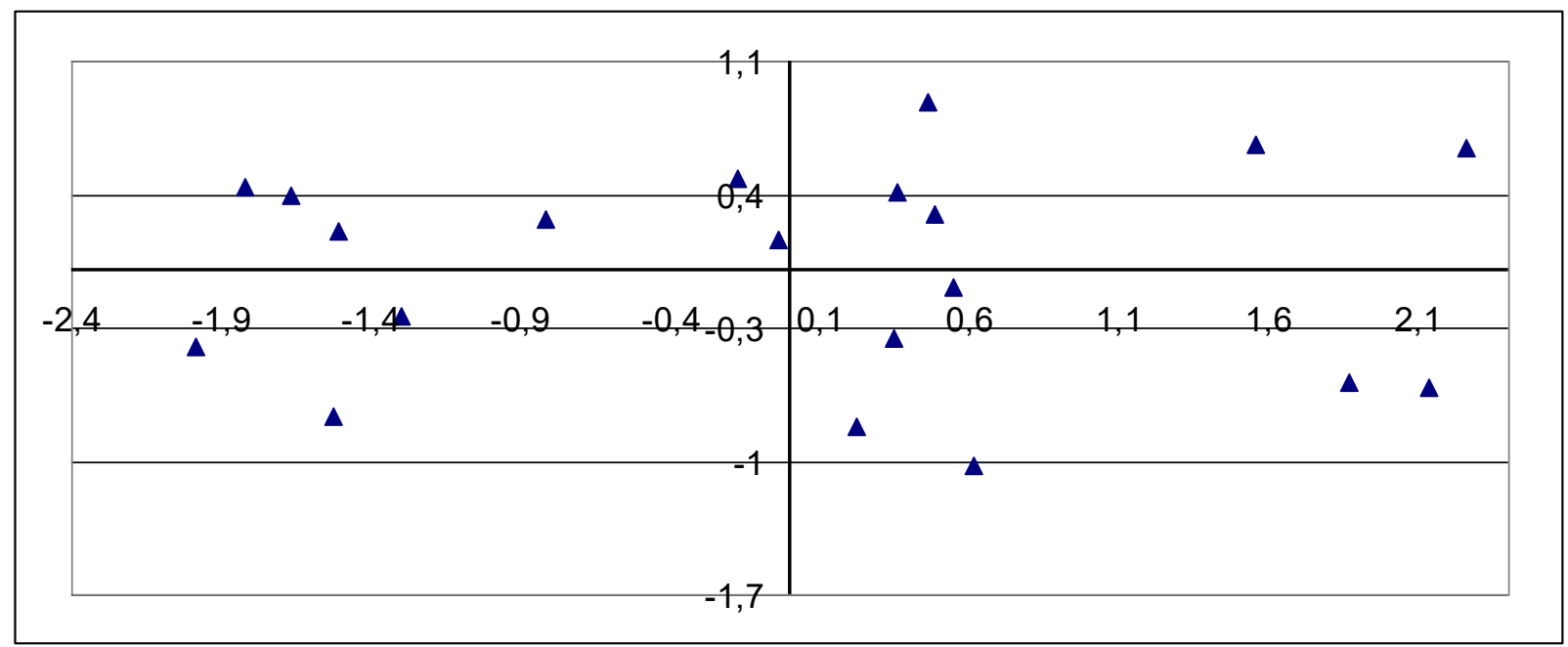

Рисунок 3. Облако точек с координатами

$\left(\mathbf{y}_{\mathrm{i} 1}, \mathbf{y}_{\mathrm{i} 2}\right), \mathrm{i}=\mathbf{1}, \ldots, \mathbf{2 0}, \boldsymbol{\Lambda}_{66}=\operatorname{diag}\left(\lambda_{1}, \ldots, \lambda_{6}\right)$ 


\begin{tabular}{llllll} 
& ISRA (India) $=\mathbf{3 . 1 1 7}$ & SIS (USA) $=\mathbf{0 . 9 1 2}$ & ICV (Poland) & $=\mathbf{6 . 6 3 0}$ \\
Impact Factor: & ISI (Dubai, UAE) $=\mathbf{0 . 8 2 9}$ & PUHЦ (Russia) $=\mathbf{0 . 1 5 6}$ & PIF (India) & $=\mathbf{1 . 9 4 0}$ \\
GIF (Australia) & $=\mathbf{0 . 5 6 4}$ & ESJI (KZ) & $=\mathbf{8 . 7 1 6}$ & IBI (India) & $=\mathbf{4 . 2 6 0}$ \\
& JIF & $\mathbf{1 . 5 0 0}$ & SJIF (Morocco) $=\mathbf{5 . 6 6 7}$ & OAJI (USA) & $\mathbf{0 . 3 5 0}$ \\
\hline
\end{tabular}

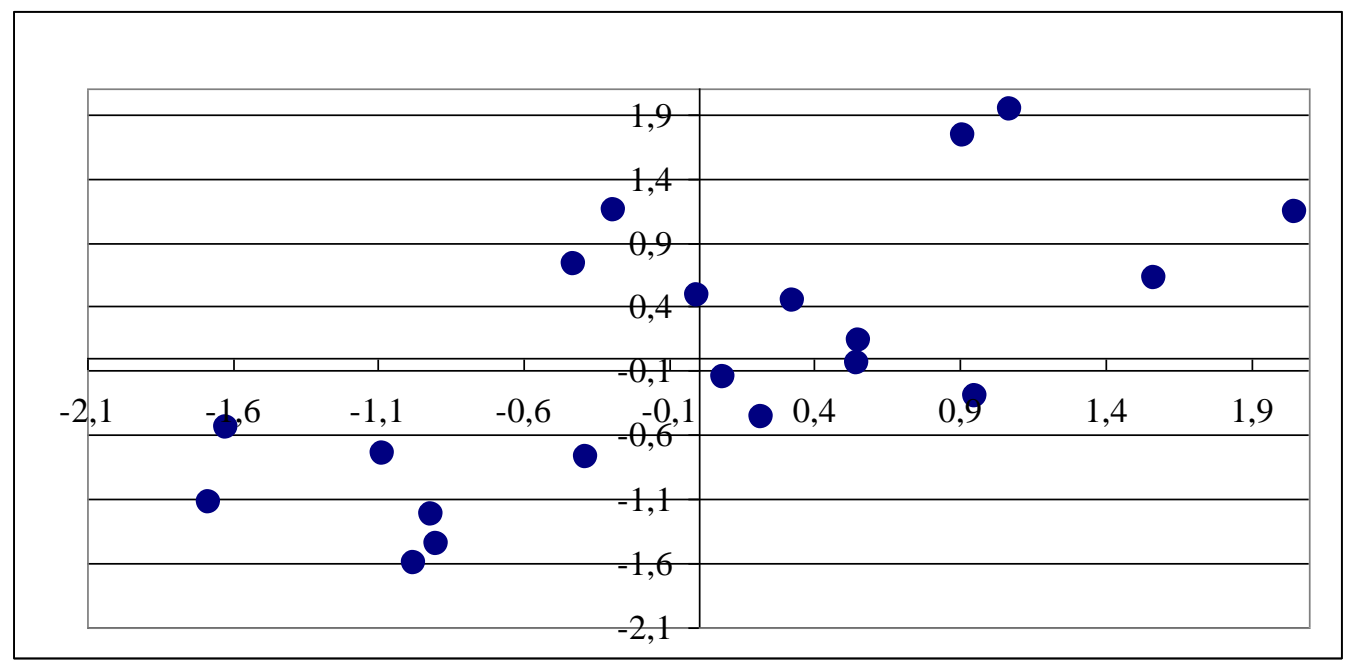

Рисунок 4. Облако точек с координатами

$\left\{\left(\mathbf{z}_{\mathbf{i} 1}, \mathbf{z}_{\mathbf{i}}\right)\right\}, \mathrm{i}=\mathbf{1}, \ldots, \mathbf{2 0}, \Lambda_{66}=\operatorname{diag}\left(\lambda_{1}, \ldots, \lambda_{6}\right)$

Схема ОС36 можно изобразить так: $\mathrm{C}_{\mathrm{nn}}=>\mathrm{C}^{+}{ }_{\mathrm{nn}}$, но при ее реализации требуется иметь начальные значения пары «реальных» матриц $\left(\mathrm{C}_{\mathrm{nn}}, \Lambda_{\mathrm{nn}}\right)$. Для этой пары матриц формулирована ниже Оптимизационная Задача.

Первая матрица после изменений значений выделенных элементов преобразуется в матрицу $\mathrm{C}^{+}{ }_{\mathrm{nn}}$, вторая - в матрицу $\mathrm{I}_{\mathrm{nn}}$. Преобразования производятся методом GRG2 в процедуре Solver с учетом ограничений $\mathrm{C}^{+\mathrm{T}}{ }_{\mathrm{nn}} \mathrm{C}^{+}{ }_{\mathrm{nn}} \neq \mathrm{I}_{\mathrm{nn}}, \mathrm{C}^{+}{ }_{\mathrm{nn}} \mathrm{C}^{+\mathrm{T}}{ }_{\mathrm{nn}}=\mathrm{I}_{\mathrm{nn}}$, $\mathbf{c}_{\mathrm{i}} \Lambda_{\mathrm{nn}} \mathbf{c}_{\mathrm{j}}^{\mathrm{T}}=\mathrm{r}_{\mathrm{ij}}$, где $\Lambda_{\mathrm{nn}}$ последовательно приближается к $\mathrm{I}_{\mathrm{nn}}, \mathrm{a} \mathrm{r}_{\mathrm{ij}}$ - к нулю в итерациях алгоритма нелинейной оптимизации Generalized Reduced Gradient (GRG2), реализованного в процедуре Solver. Процедура Solver (разработчики Леон Ласдон (Leon Lasdon, University of Texas at Austin) и Алан Уорен (Allan Waren, Cleveland State University).) используется нами при решении Оптимизационной задачи из модели ОС3 6.

$$
\mathrm{Z}_{\mathrm{mn}}=\mathrm{Y}_{\mathrm{mn}} \mathrm{C}^{+\mathrm{T}}{ }_{\mathrm{nn}}=\mathrm{U}_{\mathrm{nn}} \mathrm{C}^{+\mathrm{T}}{ }_{\mathrm{nn}},(1 / \mathrm{m}) \mathrm{U}^{\mathrm{T}}{ }_{\mathrm{mn}} \mathrm{U}_{\mathrm{mn}}=\mathrm{I}_{\mathrm{nn}}, \mathrm{C}^{+}{ }_{\mathrm{nn}} \mathrm{C}^{+}
$$
${ }_{n n n}=I_{n n}$.Преобразование (вращение) многомерной выборки $\mathrm{U}_{\mathrm{nn}}$ с некоррелированными uпеременными с помощью квадратной матрицы $\mathrm{C}^{+\mathrm{T}}{ }_{\mathrm{nn}}$, такой, что $\mathrm{C}^{+}{ }_{\mathrm{nn}} \mathrm{C}^{+\mathrm{T}}{ }_{\mathrm{nn}}=\mathrm{I}_{\mathrm{nn}}$, дает другую многомерную выборку $Z_{\mathrm{mn}}$ с некоррелированными $\mathrm{Z}$-переменными. Ее значения равны линейной комбинации u-переменных с весами, позволяющими выявлять смыслы $\mathrm{z}_{\mathrm{ij}}=\mathrm{u}_{\mathrm{i} 1} \mathrm{c}^{+}{ }_{\mathrm{j} 1}+\mathrm{u}_{\mathrm{i} 2}$ $\mathrm{c}^{+}{ }_{\mathrm{j} 2}+\ldots+\mathrm{u}_{\mathrm{in}} \mathrm{c}^{+}{ }_{\mathrm{jn}}, \mathrm{i}=1, \ldots, \mathrm{m}, \mathrm{j}=1, \ldots, \mathrm{n}$, «веса» при $\mathrm{u}-$ переменных (нормированных у-переменных) удовлетворяют условию $\mathrm{c}^{+2}{ }_{\mathrm{j} 1}+\mathrm{c}^{+2} \mathrm{j} 2_{2}+\ldots+\mathrm{c}^{+2}{ }_{\mathrm{jn}}=1$, являются весами при $\mathrm{n}$ u-переменных. Это удобно для извлечении знаний из данных. При извлечении знаний из данных мы должны опираться не на матрицу $Z_{\mathrm{mn}}$, a на матрицу другого уровня агрегирования - на пару матриц собственной структуры $\left(\mathrm{C}_{\mathrm{nn}}, \Lambda_{\mathrm{nn}}\right)$ и на преобразованную матрицу $\mathrm{Y}_{\mathrm{mn}}=\mathrm{Z}_{\mathrm{mn}} \mathrm{C}_{\mathrm{nn}}$.

В условиях ОСЗ6 имеем только одну матрицу $\mathrm{C}^{+}{ }_{n n}$, но обладающую формальными свойствами: ее строки взаимно ортогональны, элементы ј-ой строки (не ј-го собственного вектора, как было в OC31,.., ОС 35) образуют вектор единичной длины в прямоугольном базисе: $\mathrm{c}^{+2}{ }_{\mathrm{j} 1}+\mathrm{c}^{+2}{ }_{\mathrm{j} 2}+\ldots+\mathrm{c}^{+2}{ }_{\mathrm{jn}}=1$, $\mathrm{j}=1, \ldots, \mathrm{n}$. Числа $\mathrm{c}^{+2}{ }_{\mathrm{j} 1}, \mathrm{c}^{+2}{ }_{\mathrm{j} 2}, \ldots, \mathrm{c}^{+2}{ }_{\mathrm{jn}}$ являются весами при $\mathrm{n}$ u-переменных. Значения u-переменных случайны, эта случайность относится к случайности, определяемой к действительной неопределенности, а не к случайности, определяемой неполнотой знания. Такие веса позволяют, аналогично тому, как показано в работах [12-14], находить и присваивать смыслы некоррелированным Z-переменным при известном смысле u-переменной.

Ранее мы определяли зависимые когнитивные смыслы коррелированным z-переменным. Так как ОС36 моделирует некоррелированные zпеременные, то мы теперь будем вынуждены определять независимые когнитивные смыслы для некоррелированных z-переменных с весомыми весами $c_{\mathrm{kj}}$. Это потребует другого когнитивного осмысления термина «независимый смысл», употребляемый здесь как соответствующий термину «некоррелированная $\mathrm{z}$-переменная». Ранее рассматривались коррелированные z-переменные (n штук), для которых всегда существовали некоррелированные у-переменные(n штук). В ОС36 мы специально моделируем матрицу $Z_{\mathrm{mn}} \quad \mathrm{Z}-$ 


\begin{tabular}{|c|c|c|c|c|c|c|}
\hline \multirow{4}{*}{ Impact Factor: } & ISRA (India) & $=3.117$ & SIS (USA) & $=0.912$ & ICV (Poland) & $=6.630$ \\
\hline & ISI (Dubai, UAE & $=0.829$ & РИНЦ (Russia) & $=0.156$ & PIF (India) & $=1.940$ \\
\hline & GIF (Australia) & $=0.564$ & ESJI (KZ) & $=8.716$ & IBI (India) & $=4.260$ \\
\hline & JIF & $=1.500$ & SJIF (Morocco) & $=5.667$ & OAJI (USA) & $=0.350$ \\
\hline
\end{tabular}

переменных, специально моделируем матрицу $\mathrm{Y}_{\mathrm{mn}}$ y-переменных, имеющих корреляционные матрицы, равные $\quad \mathrm{I}_{\mathrm{nn}}=\operatorname{diag}(1, \ldots, 1): \quad \mathrm{Y}_{\mathrm{mn}}=\mathrm{U}_{\mathrm{nn}}, \mathrm{Z}_{\mathrm{mn}}=$ $\mathrm{Y}_{\mathrm{mn}} \mathrm{C}^{+\mathrm{T}}{ }_{\mathrm{nn}}=\mathrm{U}_{\mathrm{nn}} \mathrm{C}^{+\mathrm{T}}{ }_{\mathrm{nn}},(1 / \mathrm{m}) \mathrm{U}_{\mathrm{mn}}^{\mathrm{T}} \mathrm{U}_{\mathrm{mn}}=\mathrm{I}_{\mathrm{nn}}$, где специально решаем Оптимизационную Задачу $\mathrm{C}^{+}{ }_{n n} \mathrm{C}^{+\mathrm{T}}{ }_{n n}=\mathrm{I}_{\mathrm{nn}}$.

Что такое «фразы с «независимыми смыслами»? Можно ли формализовать конструирование «независимых смыслов? Для некоррелированных у-переменных с разными дисперсиями мы выбрали разные аспекты - четыре видения с определённых точек зрения состава показателей воздействия на индивидуальное сознание [6,10]. Ранее мы рассматривали только коррелированные $\mathrm{z}$-переменные, найденные для них зависимые смыслы приведены в [10]. Их необходимо проверить на «независимость». Теперь требуется в рамках фиксированной точки зрения найти «независимые смыслы» для некоррелированных z-переменных. Эту проблему мы рассмотрим в другой работе.

В ОС3 6 моделируются значения всех собственных чисел $\lambda^{+}{ }_{1}, \ldots, \lambda_{\mathrm{n}}^{+} \quad$ и псевдособственные векторы $\mathbf{c}^{+}{ }_{\mathbf{j}}=\left(\mathrm{c}^{+}{ }_{1 \mathrm{j}}, \mathrm{c}^{+}{ }_{2 \mathrm{j}} \ldots \mathrm{c}^{+}{ }_{\mathrm{nj}}\right)^{\mathrm{T}}$, $\mathrm{j}=1, \ldots, \mathrm{n}, \quad$ из матрицы $\mathrm{C}^{+}{ }_{\mathrm{nn}}=\left[\mathrm{c}^{+}{ }_{1}|\ldots| \mathrm{c}^{+}{ }_{\mathrm{n}}\right] . \quad$ При переменных значениях всех собственных чисел $\lambda_{1}, \ldots, \lambda_{\ell}$ и при переменных компонентах собственных векторов $\mathbf{c}_{\mathbf{j}}=\left(\mathrm{c}_{1 \mathrm{j}}, \mathrm{c}_{2 \mathrm{j}} \ldots \mathrm{c}_{\mathrm{nj}}\right)^{\mathrm{T}}, \mathrm{j}=1, \ldots, \mathrm{n}$, из матрицы $\mathrm{C}_{\mathrm{nn}}=\left[\mathbf{c}_{1}|. ..| \mathbf{c}_{\mathbf{n}}\right]$. Элементы матрицы $\mathrm{C}_{\mathrm{nn}}=\left[\mathbf{c}_{1}|..| \mathbf{c}_{\mathbf{n}}\right]$ в ОСЗ6 служат переменными решаемой ниже Оптимизационной задачи, таковыми являются и значения собственных чисел $\lambda_{1}, \ldots, \lambda_{\ell}$, таких, что $\lambda_{1}>\ldots>\lambda_{\ell}>0$. Элементы матрицы собственных чисел должны удовлетворять одному условию: $\left.\mathrm{f}_{1}\left(\Lambda_{\mathrm{nn}}\right)=\lambda_{1}+\lambda_{2}+\ldots+\lambda_{\mathrm{n}}\right)=\mathrm{n}$.

Это условие означает существование корреляционной матрицы $\operatorname{diag}\left(\mathrm{R}_{\mathrm{nn}}\right)=(1, \ldots, 1)$, $\operatorname{tr}\left(R_{n n}\right)=1+\ldots+1=\operatorname{tr}\left(\Lambda_{n n}\right)=\lambda_{1}+\ldots+\lambda_{n}=n, \quad \lambda_{1} \geq \ldots \geq \lambda_{n} \geq 0$. Отсутствие ограничений на значения f-параметров $\mathrm{f}_{2}, \mathrm{f}_{3}, \mathrm{f}_{4}, \mathrm{f}_{5}, \mathrm{f}_{6}$, как убедимся ниже, приведет к ситуации существования некорелированных zпеременных с теми же смыслами, что были когнитивно найдены в работе [4, Таблица 3] для корелированных z-переменных. Некорелированные z-переменные, получаемые преобразованием некорелированных у-переменных должны иметь другие смысли, отличающиеся от смыслов из [6,Таблица 3]. Нахождением указанных новых смыслов мы займемся в другой работе. Здесь ограничимся «математическим открытием» теоретического существования таких некорелированных z-переменных путем использования процедуры Solver при решении сформулированной ОС3 6.

Оптимизационная задача для модели ОСЗ 6
Оптимизационная Задача (OC3 6): Для известных значений собственных чисел $\lambda_{1}, \ldots, \lambda_{\mathrm{n}}$, $\Lambda_{\mathrm{nn}}=\operatorname{diag}\left(\lambda_{1}, \ldots, \lambda_{\mathrm{n}}\right)$ таких, что $\lambda_{1}>\ldots>\lambda_{\mathrm{n}}>0$ и известных и заданных значений некоторых компонент $\mathrm{y} n$ собственных векторов $\mathbf{c}_{1}, \ldots, \mathbf{c}_{\mathbf{n}}$ из матрицы $\mathrm{C}_{\mathrm{nn}}=\left[\mathbf{c}_{1}|\ldots| \mathbf{c}_{\mathbf{n}}\right]$ собственных векторов найти:

1)новые модельные значения известным, но не заданным значениям компонент $\mathrm{n}$ псевдо собственных векторов $\quad \mathbf{c}^{+} \boldsymbol{\ell}+\mathbf{1}, \ldots, \mathbf{c}^{+}$, $\mathbf{c}^{+}{ }_{\mathrm{j}}=\left(\mathrm{c}^{+}{ }_{1 \mathrm{j}}, \mathrm{c}^{+}{ }_{2 \mathrm{j}} \ldots \mathrm{c}^{+}{ }_{\mathrm{nj}}\right)^{\mathrm{T}}, \quad \mathrm{j}=1, \ldots, \mathrm{n}, \quad$ из новой матрицы $\mathrm{C}^{+}{ }_{\mathrm{nn}}=\left[\mathbf{c}^{+}{ }_{1}|\ldots| \mathbf{c}^{+}{ }_{\mathrm{n}}\right]$ псевдособственных векторов;

2)полученная матрица собственных чисел $\Lambda^{+}{ }_{n n}=\operatorname{diag}(1, \ldots,, 1)$ должна иметь значения, равные 1 : $\Lambda^{+}{ }_{\mathrm{nn}}=\operatorname{diag}(1, \ldots,, 1)=\mathrm{I}_{\mathrm{nn}}$.

3)полученные полные матрица собственных чисел $\Lambda_{66}^{+}=\mathrm{I}_{\mathrm{nn}} \quad$ и матрица псевдособственных векторов $\mathrm{C}^{+}{ }_{n n}=\left[\mathbf{c}^{+} 1|\ldots| \mathbf{c}^{+}\right]$должны удовлетворять равенствам

$\mathrm{C}^{+\mathrm{T}}{ }_{n n} \mathrm{C}^{+}{ }_{n n} \neq \mathrm{I}_{\mathrm{nn}}, \mathrm{C}^{+}{ }_{\mathrm{nn}} \mathrm{C}^{+\mathrm{T}}{ }_{\mathrm{nn}}=\mathrm{I}_{\mathrm{nn}}, \mathrm{C}^{+}{ }_{\mathrm{nn}} \Lambda^{+}{ }_{\mathrm{nn}} \mathrm{C}^{+\mathrm{T}}{ }_{\mathrm{nn}}=\mathrm{I}_{\mathrm{nn}}$, $\mathbf{c}_{\mathrm{j}}{ }^{+} \Lambda^{+}{ }_{\mathrm{nn}} \mathbf{c}_{\mathrm{j}}{ }^{+\mathrm{T}}=1, \mathbf{c}_{\mathrm{i}}^{+} \Lambda^{+}{ }_{66} \mathbf{c}_{\mathrm{j}}{ }^{+\mathrm{T}}=\mathrm{r}^{+}{ }_{\mathrm{ij}}=0, \mathrm{r}^{+}{ }_{\mathrm{ij}}=\mathrm{r}^{+}{ }_{\mathrm{ji}}=0, \mathrm{i}=1, \ldots, \mathrm{n} ; \mathrm{j}=1$, $\ldots, \mathrm{n}, \mathrm{i} \neq \mathrm{j}$, а корреляционная матрица $\mathrm{R}^{+}{ }_{n n}=\mathrm{C}^{+}{ }_{n n} \Lambda^{+}{ }_{66} \mathrm{C}^{+\mathrm{T}}{ }_{\mathrm{nn}}=\mathrm{I}_{\mathrm{nn}}$ должна иметь новые матрицы псевдособственных векторов $\mathrm{C}^{+}{ }_{\mathrm{nn}} \neq \mathrm{I}_{\mathrm{nn}} \quad$ и собственных чисел $\Lambda^{+}{ }_{\mathrm{nn}}=\mathrm{I}_{\mathrm{nn}}=\operatorname{diag}(1, \ldots, 1), \lambda^{+}{ }_{1}=\ldots=$ $\lambda^{+}{ }_{\mathrm{n}}=1, \lambda_{1}+\ldots+\lambda^{+} \mathrm{n}=\mathrm{n}$.

Условие

неравенства матрицы псевдособственных векторов единичной матрице: $\mathrm{C}^{+}{ }_{n n} \quad \neq \mathrm{I}_{\mathrm{nn}}$ при наличии соответствующей ей единичной матрицы собственных чисел $\Lambda^{+}{ }_{n n}=I_{n n}$ является нетрадиционным требованием. Обычно в известной ПСЗ: $\mathrm{R}_{\mathrm{nn}}=>\left(\Lambda_{\mathrm{nn}}, \mathrm{C}_{\mathrm{nn}}\right)$ имеет место равенство $\mathrm{R}_{\mathrm{nn}} \mathrm{C}_{\mathrm{nn}}=\mathrm{C}_{\mathrm{nn}} \Lambda_{\mathrm{nn}}$ и, если $\mathrm{R}_{\mathrm{nn}}=\mathrm{I}_{\mathrm{nn}}$, то $\mathrm{C}_{\mathrm{nn}}=\mathrm{I}_{\mathrm{nn}}$. В OC3 6 мы решаем обратную спектральную задачудля заданной матрицы собственных чисел $\Lambda^{+}{ }_{\mathrm{nn}}=\mathrm{I}_{\mathrm{nn}}=\operatorname{diag}(1, \ldots, 1), \quad \lambda^{+}{ }_{1}=\ldots=\lambda^{+}{ }_{\mathrm{n}}=1, \quad \lambda_{1}+\ldots+\lambda^{+}{ }_{\mathrm{n}}=\mathrm{n}$, найти матрицу «псевдособственных» векторов $\mathrm{C}^{+}{ }_{\mathrm{nn}}$ такую, что $\mathrm{C}^{+\mathrm{T}}{ }_{n n} \mathrm{C}^{+}{ }_{n n} \neq \mathrm{I}_{\mathrm{nn}}, \mathrm{C}^{+}{ }_{\mathrm{nn}} \mathrm{C}^{+\mathrm{T}}{ }_{\mathrm{nn}}=\mathrm{I}_{\mathrm{nn}}$. Как показано ниже для ОС 6 существует нетривиальное решение $\mathrm{C}^{+}{ }_{\mathrm{nn}} \neq \mathrm{I}_{\mathrm{nn}}$, отличающееся от решения соответствующей нашей обратной задаче прямой задачи (ПСЗ), причем не единственное. Применение решения $\mathrm{C}^{+}{ }_{\mathrm{nn}} \neq \mathrm{I}_{\mathrm{nn}}$ будет изложено в отдельной статье.

Модель ОСЗ 6 схематически изображается так: $\left(\Lambda_{\mathrm{nn}}, \mathrm{C}_{\mathrm{nn}}\right)=>\left(\Lambda_{\mathrm{nn}}, \mathrm{C}^{0}{ }_{\mathrm{nn}}\right)=>\left(\mathrm{C}^{+}{ }_{\mathrm{nn}}, \mathrm{I}^{+}{ }_{\mathrm{nn}}\right)$. Верхний индекс $\left({ }^{0}\right)$ в обозначении матрицы $\mathrm{C}^{0}{ }_{n n}$ означает наличие выделенных компонент с назначенными пользователем (с заданными) значениями компонент некоторых собственных векторов.

Для матрицы $\mathrm{C}^{0}{ }_{\mathrm{nn}}$ (с новыми назначенными значениями $\left.\mathrm{c}^{0}{ }_{\mathrm{kj}}, \mathrm{j} \in 1, \ldots, \mathrm{n}, \mathrm{k} \in\{1, \ldots, \mathrm{n}\}\right)$ требуется найти новую пару матриц $\left(\mathrm{C}^{+}{ }_{\mathrm{nn}}, \Lambda^{+}{ }_{\mathrm{nn}}=\mathrm{I}_{\mathrm{nn}}\right)$, такую, что матрица $\mathrm{C}^{+}{ }_{n n}$ имеет те же заданные пары индексов $(\mathrm{k}, \mathrm{j})$ и те же новые значения компонентов $\mathrm{c}^{+} \mathrm{kj}$, $\mathrm{j}=1, \ldots, \mathrm{n}, \mathrm{k} \in\{1, \ldots, \mathrm{n}\}$, что и у $\mathrm{n}$ псевдособственных векторов $\mathbf{c}^{+}=\left(\mathrm{c}^{+}{ }_{1 j}, \mathrm{c}^{+}{ }_{2 j} \ldots \mathrm{c}^{+}{ }_{n j}\right)^{\mathrm{T}}, \quad$ расположенных по столбцам матрицы $\mathrm{C}^{+}{ }_{\mathrm{nn}}=\left[\mathbf{c}^{+}{ }_{1}|\ldots| \mathbf{c}_{\mathrm{n}}^{+}\right]$. В матрице $\mathrm{C}_{\mathrm{nn}}^{0}$ 


\begin{tabular}{|c|c|c|c|c|c|c|}
\hline \multirow{4}{*}{ Impact Factor: } & ISRA (India) & $=3.117$ & SIS (USA) & $=0.912$ & ICV (Poland) & $=6.630$ \\
\hline & ISI (Dubai, UAE & $=\mathbf{0 . 8 2 9}$ & РИНЦ (Russia & $=0.156$ & PIF (India) & $=1.940$ \\
\hline & GIF (Australia) & $=0.564$ & ESJI (KZ) & $=8.716$ & IBI (India) & $=4.260$ \\
\hline & JIF & $=1.500$ & SJIF (Morocco & $=5.667$ & OAJI (USA) & $=0.350$ \\
\hline
\end{tabular}

могут присутствовать или могут отсутствовать заданные пользователем значения выделенных компонент у собственных векторов.

Решение Оптимизационной задачи для модели OC3 6 проведем с помощью процедуры Solver. B окне процедуры Solver в строке «Изменяемые ячейки» важен порядок адресов, где располагаются переменные нашей Оптимизационной задачи: сперва вставляем адреса ячеек с значениями матрицы $\mathrm{C}_{66}^{0}$, затем через знак (;) - адреса ячеек с значениями $\lambda_{1}=1.75, \lambda_{2}=1.38373, \lambda_{3}=1,3663, \lambda_{4}=1$, $\lambda_{5}=0,3, \lambda_{6}=0,2$.

Формулировка задачи из модели ОС3 6 отличается от формулировки задачи из модели ОС3 2 отсутствием разбиения матрицы $\mathrm{C}^{+}$n. В ПСЗ матрицы $\mathrm{C}^{+}{ }_{\mathrm{nn}}$ и $\Lambda^{+}{ }_{\mathrm{nn}}=\mathrm{I}_{\mathrm{nn}}$ должны удовлетворять равенствам:

$\mathrm{C}^{+\mathrm{T}}{ }_{\mathrm{nn}} \mathrm{C}^{+}{ }_{\mathrm{nn}}=\mathrm{C}^{+}{ }_{\mathrm{nn}} \mathrm{C}^{+\mathrm{T}}{ }_{\mathrm{nn}}=\mathrm{I}_{\mathrm{nn}}, \mathrm{C}^{+}{ }_{\mathrm{nn}} \Lambda^{+}{ }_{\mathrm{nn}} \mathrm{C}^{+\mathrm{T}}{ }_{\mathrm{nn}}=\mathrm{R}^{+}{ }_{\mathrm{nn}}$,

$\lambda^{+}{ }_{1}+\ldots+\lambda^{+}{ }_{n}=n, c_{j}{ }^{+} \Lambda^{+}{ }_{n n} \mathbf{c}_{j}{ }^{+\mathrm{T}}=1, \mathbf{c}_{\mathrm{j}}{ }^{+\mathrm{T}} \mathbf{c}_{\mathrm{j}}{ }^{+} \neq 1, \mathbf{c}_{\mathrm{i}}{ }^{+} \Lambda^{+}{ }_{\mathrm{nn}} \mathbf{c}_{\mathrm{j}}{ }^{+\mathrm{T}}=\mathrm{r}^{+} \mathrm{ij}$,

$\mathrm{r}^{+}{ }_{\mathrm{ij}}=\mathrm{r}_{\mathrm{ji}}^{+} \mathrm{i}=1, \ldots, \mathrm{n} ; \mathrm{j}=1, \ldots, \mathrm{n}, \quad$ где корреляционная матрица $\mathrm{R}^{+}{ }_{\mathrm{nn}}$ имеет новые матрицы псевдособственных векторов $\mathrm{C}^{+}{ }_{\mathrm{nn}}=\left[\mathrm{c}_{1}|\ldots| \mathrm{c}_{\mathrm{n}}\right]$, и собственных чисел $\Lambda^{+}{ }_{n n}=I_{n n}=\operatorname{diag}\left(\lambda_{1}, \ldots, \lambda_{n}\right)$.

В модели ОС3 6: $\left(\Lambda_{\mathrm{nn}}, \mathrm{C}_{\mathrm{nn}}\right)=>\left(\mathrm{C}_{\mathrm{nn}}^{0}\right]$, $\left.\Lambda_{\mathrm{nn}}\right)=>\left(\mathrm{C}^{+}{ }_{\mathrm{nn}}, \Lambda^{+}{ }_{\mathrm{nn}}\right)$ не требуется равенства 1 длин псевдособственных векторов $\mathbf{c}_{\mathrm{j}}^{+\mathrm{T}} \mathbf{c}_{\mathrm{j}}^{+} \neq 1, \mathrm{j} \in\{1, \ldots, \mathrm{n}\}$. Решения OC3 6: пары матриц $\mathrm{I}_{\mathrm{nn}}, \mathrm{C}^{+}{ }_{\mathrm{nn}}$ применены при реализации ОМ ГК: $\left(\mathrm{C}^{+}{ }_{\mathrm{nn}}, \mathrm{I}_{\mathrm{nn}}\right)=>\left(\mathrm{R}^{+}{ }_{\mathrm{nn}}, \mathrm{Z}^{(\mathrm{t})}{ }_{\mathrm{mn}}, \mathrm{Y}^{(\mathrm{t})}{ }_{\mathrm{mn}}\right), \mathrm{t}=1, \ldots, \mathrm{k}_{\mathrm{t}}<\propto$.

Также как и в модели ОСЗ3 в программетаблице из ОСЗ6 важен порядок следования изменяемых ячеек программы. Реализация модели OC3 6 требует такой порядок перечисления изменяемых ячеек: $\mathrm{C}_{66}^{+}, \lambda_{1}^{+}, \lambda^{+}{ }_{2}, \ldots, \lambda_{6}^{+}$. Требуется назначение $\mathrm{n}^{2}$ элементов в панели «Изменяемые ячейки» процедуры Solver, затем ввод адресов $\mathrm{n}$ ячеек с $\lambda_{1}, \ldots, \lambda_{6}$. В противном случае процедура «выдает» неверное решение.

\section{Программа-таблица ОС3 6}

Основные шаги модели ОСЗ 6 приведены выше. Приведем описание программы-таблицы модели ОС3 6 при $\mathrm{n}=6$. В Таблице 1 приводим начальные значения компонент собственных векторов из известной исходной матрицы $\mathrm{C}_{66}$. Назначенные значения выделенных компонент в ней не видны, они появляются после нажатия кнопки «Выполнить». В нижней части Таблицы 1 приведены начальные значения элементов спектра Old $\Lambda_{66}=\operatorname{diag}(1.75,1.38373,1.3663,1,0.3,0.2)$, которые после итераций в методе GRG2 в процедуре Solver последовательно $\mathrm{I}_{\mathrm{nn} .}=\operatorname{diag}(1,1,1,1,1,1)$.

Эта пара $\left(\mathrm{C}_{66}, \Lambda_{66}\right)$ входных объектов модели ОС36. Открыв окно процедуры «Поиск решения» дополнительно вводим в ячейки программытаблицы формулы из модели ОСЗ6 и числовые значения (0.5 или 0.65) выделенных компонент собственных векторов из известной исходной матрицы $\mathrm{C}_{66}$. В Таблице 2- в программе-таблице вычисления пары матриц $\left(\mathrm{C}_{66}^{+}, \mathrm{I}_{66}\right)$ в колонке «Summa squares» выделены красным цветом значения 1.0000, полученные из соотношения $\mathrm{C}^{+}{ }_{\mathrm{nn}} \mathrm{C}^{+\mathrm{T}}{ }_{\mathrm{nn}}=\mathrm{I}_{\mathrm{nn}}$. Отметим отдельно: для всех 6-ти собственных векторов, применяемых нами ниже, в окне процедуры Solver (Рисунок 1) не вводим ограничения для элементов матрицы $\mathrm{C}^{+}{ }_{\mathrm{nn}}$ вида $\mathrm{C}^{+\mathrm{T}} \mathrm{C}^{+}{ }_{n n}=\mathrm{I}_{\mathrm{nn}}$. На Рисунке 2 приведено Окно параметров надстройки «Поиск решения» для программы-таблицы из Таблицы 1 (Таблицы 2).

Матрица $\mathrm{C}_{66}^{+}$и она является матрицей преобразования 6-мерной выборки $\mathrm{Y}_{\mathrm{m} 6}=\mathrm{U}_{\mathrm{n} 6}$ в 6мерной выборку $\mathrm{Z}_{\mathrm{m} 6}=\mathrm{U}_{\mathrm{m} 6}\left[\mathrm{C}^{+}{ }_{66}\right]^{\mathrm{T}}$ стандартизованных Z-переменных с многомерного распределения.

Таблица 1. Начальные значения компонент собственных векторов из известной исходной матрицы Сбб (фрагмент программы-таблицы)

\begin{tabular}{|c|c|c|c|c|c|c|c|}
\hline & eigen vector $c_{\cdot 1}$ & eigen vector $c_{.2}$ & eigen vector $c_{.3}$ & eigen vector $c_{.4}$ & eigen vector $c_{.5}$ & $\begin{array}{c}\text { eigen vector } \\
c_{.6} \\
\end{array}$ & Summa squares \\
\hline ROW 1 & 0,5106 & $-0,3477$ & $-0,6143$ & $-0,3411$ & 0,2672 & $-0,2307$ & 0,9999 \\
\hline ROW 2 & 0,0665 & $-0,4642$ & $-0,0354$ & 0,3835 & $-0,6793$ & $-0,4128$ & 1,0001 \\
\hline ROW 3 & 0,4569 & $-0,3185$ & 0,0972 & 0,4628 & 0,109 & 0,674 & 1,0000 \\
\hline ROW 4 & 0,2923 & $-0,6128$ & 0,2572 & $-0,5981$ & $-0,1469$ & 0,306 & 1,0001 \\
\hline ROW 5 & 0,5129 & 0,421 & 0,072 & $-0,3978$ & $-0,5934$ & 0,21 & 1,0000 \\
\hline \multirow[t]{2}{*}{ ROW 6} & 0,4215 & $-0,0976$ & 0,7352 & $-0,0796$ & 0,2856 & $-0,4294$ & \multirow[t]{2}{*}{1,0000} \\
\hline & 1,0001 & 1,00011 & 0,9999 & 0,9999 & 1,0000 & 1,0000 & \\
\hline Old $\Lambda_{66}$ & 1,75 & 1,38373 & 1,3663 & 1 & 0,3 & 0,2 & 6 \\
\hline
\end{tabular}




\begin{tabular}{llllll} 
& ISRA (India) $=\mathbf{3 . 1 1 7}$ & SIS (USA) $=\mathbf{0 . 9 1 2}$ & ICV (Poland) & $\mathbf{= 6 . 6 3 0}$ \\
Impact Factor: & ISI (Dubai, UAE) $=\mathbf{0 . 8 2 9}$ & PUHЦ (Russia) $=\mathbf{0 . 1 5 6}$ & PIF (India) & $=\mathbf{1 . 9 4 0}$ \\
GIF (Australia) & $=\mathbf{0 . 5 6 4}$ & ESJI (KZ) & $=\mathbf{8 . 7 1 6}$ & IBI (India) & $=\mathbf{4 . 2 6 0}$ \\
& JIF & $\mathbf{1 . 5 0 0}$ & SJIF (Morocco) $=\mathbf{5 . 6 6 7}$ & OAJI (USA) & $\mathbf{0 . 3 5 0}$ \\
\hline
\end{tabular}

Таблица 2. Программа-таблица вычисления пары матриц $\left(\mathrm{C}^{+}{ }_{66}, \mathrm{I}_{66}\right)$ из модели ОСЗ 6 с заданными значениями выделенных элементов из $\mathrm{C}^{+} 6$

\begin{tabular}{|c|c|c|c|c|c|c|c|}
\hline & $\begin{array}{c}\text { eigen vector } \\
c_{.} \cdot 1\end{array}$ & $\begin{array}{c}\text { eigen vector } \\
\text { c.2 }_{2}\end{array}$ & $\begin{array}{c}\text { eigen vector } \\
\text { c.3 }_{3}\end{array}$ & $\begin{array}{c}\text { new eigen vector } \\
\mathbf{c}_{\cdot 4}\end{array}$ & $\begin{array}{c}\text { new eigen vector } \\
\text { c.5 }^{-5}\end{array}$ & $\begin{array}{c}\text { new eigen vector } \\
\mathrm{c}_{.6} .\end{array}$ & $\begin{array}{l}\text { Summa } \\
\text { squares }\end{array}$ \\
\hline ROW 1 & $-4,08 \mathrm{E}-08$ & $-0,5$ & $-0,65$ & $-4,29 \mathrm{E}-08$ & $-2 \mathrm{E}-09$ & $-0,5722762$ & 1,0000 \\
\hline ROW 2 & $-4,34 \mathrm{E}-08$ & $-0,5$ & $-0,024482$ & 0,65 & 0,571752 & 3,8876E-07 & 1,0000 \\
\hline ROW 3 & 0,5 & $-0,5$ & 0,146982 & 0,5 & 0,477908 & $-1,264 \mathrm{E}-05$ & 1,0000 \\
\hline ROW 4 & 0 & $-0,5$ & 0,235153 & $-0,5$ & $-0,66686$ & $1,5107 \mathrm{E}-05$ & 1,0000 \\
\hline ROW 5 & 0,65 & $\begin{array}{r}\mathbf{0 , 5} \\
-4,882 \mathrm{E}-\end{array}$ & 0,2332724 & $-0,422$ & $-1 \mathrm{E}-04$ & 0,3082207 & 1,0000 \\
\hline \multirow[t]{2}{*}{ ROW 6} & $-5,02 \mathrm{E}-08$ & 08 & 0,65 & $-5 \mathrm{E}-08$ & $1,25 \mathrm{E}-07$ & 0,75993421 & 1,0000 \\
\hline & 0,6725 & 1,25000 & 0,9769 & 1,1006 & 1,0000 & 1,0000 & \\
\hline New $\Lambda_{66}$ & 1 & 1 & 1 & 1 & 1 & 1 & 6 \\
\hline Old $\Lambda_{66}$ & 1,75 & 1,38373 & 1,3663 & 1 & 0,3 & 0,2 & \\
\hline
\end{tabular}

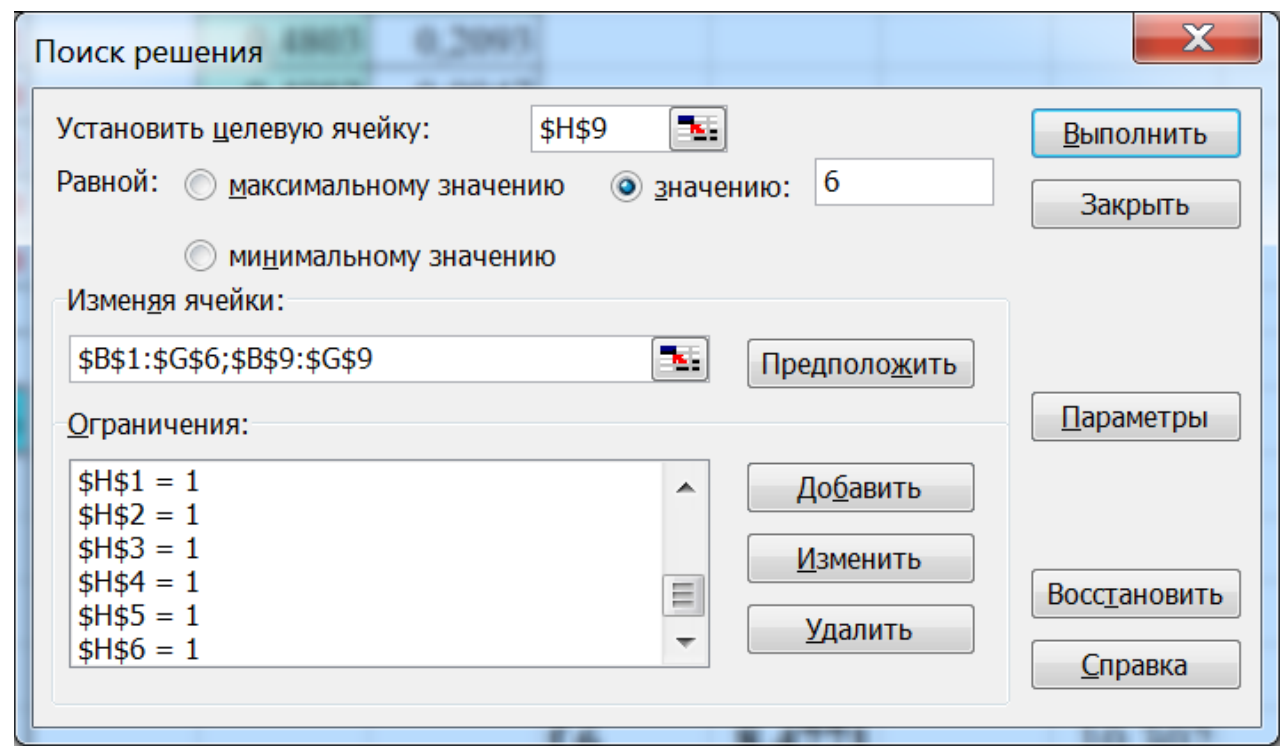

Рисунок 1. Окно надстройки «Поиск решения» для программы-таблицы из Таблицы 2 


\begin{tabular}{|c|c|c|c|c|c|c|}
\hline \multirow{4}{*}{ Impact Factor: } & ISRA (India) & $=3.117$ & SIS (USA) & $=0.912$ & ICV (Poland) & $=6.630$ \\
\hline & ISI (Dubai, UAE & $=0.829$ & РИНЦ (Russia & $=0.156$ & PIF (India) & $=1.940$ \\
\hline & GIF (Australia) & $=0.564$ & ESJI (KZ) & $=8.716$ & IBI (India) & $=4.260$ \\
\hline & JIF & $=1.500$ & SJIF (Morocco & $=5.667$ & OAJI (USA) & $=0.350$ \\
\hline
\end{tabular}

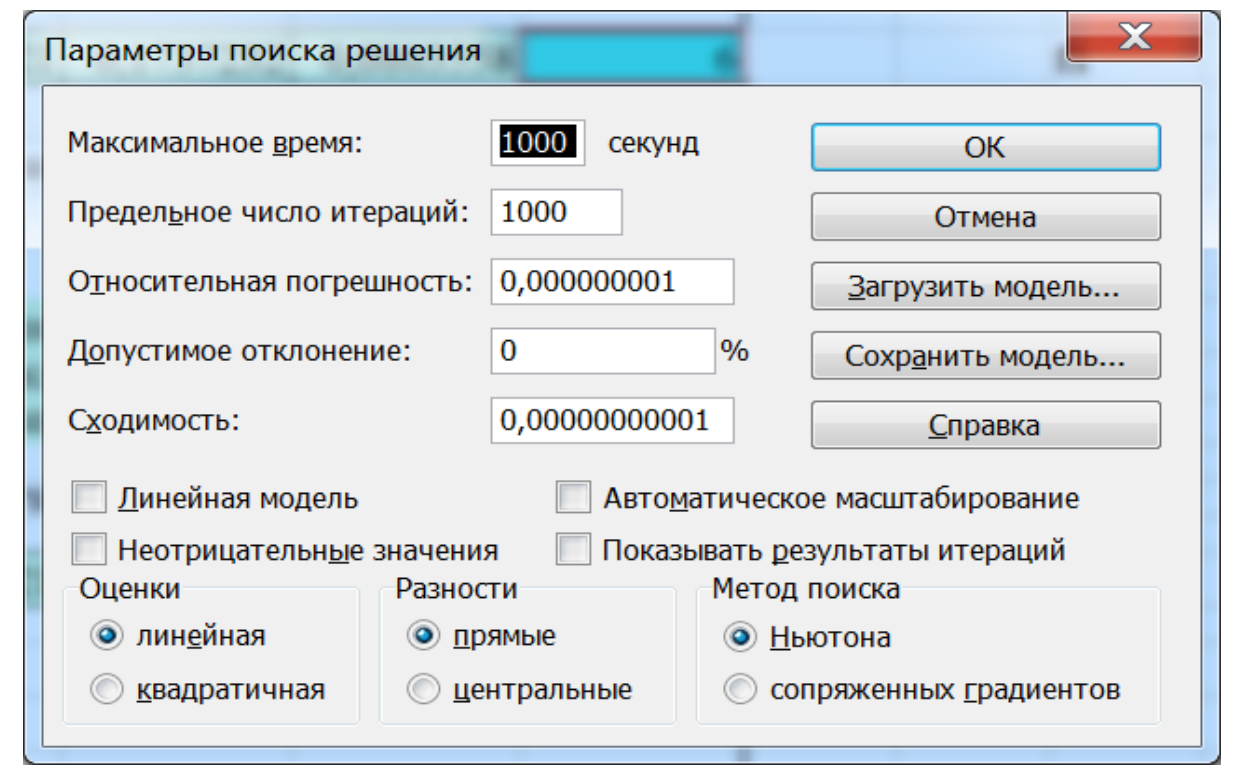

Рисунок 2. Окно параметров надстройки «Поиск решения» для программы-таблицы из Таблицы 1

\section{Заключение}

Мы разработали модификации и приложения моделей ОСЗ, и новые задачи моделирования зависимостей модельной матрицы псевдособственных векторов $\mathrm{C}^{+}{ }_{\mathrm{nn}}$, независимой от спектра симметрической корреляционной матрицы. Рассмотрели новый термин «коэффициент комбинационных пропорциональностей» и интерпретацию значения «коэффициента комбинационных пропорциональностей». В моделях ОСВ4,ОС 35 , ОС36 сформулировали и решили Оптимизационные Задачи с соответствующими допущениями на их параметры и переменные.

Формализовали процесс конструирования показателей, соответствующих «независимым смыслам. Показали, что в модели ОС36 матрица псевдособственных векторов неединичной длины $\mathrm{C}^{+}{ }_{n n}$ является (в когнитивных моделях извлечения цифровых знаний, в когнитивных алгоритмах придания смыслов) матрицей значений коэффициентов комбинационных пропорциональностей при значениях изменчивостей некоррелированных $\mathrm{z}-$ переменных. А матрица $Z_{\mathrm{mn}}$ является матрицей значений коэффициентов изменчивостей $\mathrm{z}-$ переменных. Матрица псевдособственных векторов $\mathrm{C}^{+}$nn доминирует при когнитивном извлечении цифровых знаний из многомерной выборки $\quad \mathrm{Z}_{\mathrm{mn}}=\mathrm{U}_{\mathrm{mn}}\left[\mathrm{C}^{+}{ }_{\mathrm{nn}}\right]^{\mathrm{T}}$ имеет модельную матрица «весов» $\mathrm{C}^{+}{ }_{\mathrm{nn}}$. Получен ответ на вопрос: в новых Обратных Спектральных Задачах что первично $\mathrm{C}_{\mathrm{nn}}$ или $\Lambda_{\mathrm{nn}}$ ? или что мы должны иметь в качестве входного объекта в моделях ОС3? Получен обоснованный ответ: это зависит от решаемой обратной задачи. Если мы решаем задачу моделирования многомерной выборки, адекватной многомерной реальной выборке, то входным объектом может быть пара $\left(\mathrm{C}_{\mathrm{nn}}, \Lambda_{\mathrm{nn}}\right)$. Если мы решаем задачу моделирования многомерной выборки, содержащей цифровые знания, извлекаемые с применением когнитивного моделирования [6-9], то входным объектом может быть матрица псевдособственных векторов $\mathrm{C}^{+}{ }_{\mathrm{nn}}$.

В модели ОС36 произведена трансформация ролей матриц собственных чисел и собственных матриц. матрица $\mathrm{C}^{+}{ }_{\mathrm{nn}}$ содержит вместо собственных векторов псевдособственные векторы. Введенные новые термины необходимы для озвучивания смысла у-переменной, равной линейной комбинации $\mathrm{y}_{\mathrm{ij}}=\mathrm{z}_{\mathrm{i} 1} \mathrm{c}_{1 \mathrm{j}}+\mathrm{z}_{\mathrm{i} 2} \mathrm{c}_{2 \mathrm{j}} \ldots+\mathrm{z}_{\mathrm{in}} \mathrm{c}_{\mathrm{nj}}$, где только $\ell$ «весов» удовлетворяют критериям «существенности», «значимости». Чем больше число $\ell$, тем труднее формулируется смысл линейной комбинации вида $\mathrm{y}_{\mathrm{ij}}=\mathrm{z}_{\mathrm{i} 1} \mathrm{c}_{1 \mathrm{j}}+\mathrm{z}_{\mathrm{i} 2}$ $\mathrm{c}_{2 \mathrm{j}}+\ldots+\mathrm{z}_{\mathrm{in}} \mathrm{c}_{\mathrm{nj}}$ (валидной переменной). В $[1,6]$ приведены когнитивные модели выявления смысла суммы значимых (весомых) переменных для известных смыслов $\ell$ штук z-переменных. В $[4,6]$ приведены когнитивные модели выявления неизвестных смыслов $\ell$ z-переменных, входящих в $\mathrm{y}$-переменную, состоящей из линейной комбинации значимых (весомых) z-переменных. В этих 2-х классах когнитивных моделей рассматриваются взаимно обратные когнитивные 


\begin{tabular}{|c|c|c|c|c|c|c|}
\hline \multirow{4}{*}{ Impact Factor: } & ISRA (India) & $=\mathbf{3 . 1 1 7}$ & SIS (USA) & $=0.912$ & ICV (Poland) & $=6.630$ \\
\hline & ISI (Dubai, UAE & $=0.829$ & РИНЦ (Russia & $=0.156$ & PIF (India) & $=1.940$ \\
\hline & GIF (Australia) & $=0.564$ & ESJI (KZ) & $=8.716$ & IBI (India) & $=4.260$ \\
\hline & JIF & $=1.500$ & SJIF (Morocco & $=5.667$ & OAJI (USA) & $=0.350$ \\
\hline
\end{tabular}

задачи: первая - задача извлечения цифрового знания из цифровых данных, вторая - задача цифровизации $\mathrm{z}$-переменных при известных смыслах $\ell$ валидных показателей (у-переменных).

Матрицы $\mathrm{C}^{+}{ }_{n n}$ в ОС36 таковы, что в них количество обладающих весомыми (значимыми) «весами» $у$-переменных равно $\ell$. Количество значимых «весов» у ј-ой у-переменной не зависит от значения $\lambda_{\mathrm{j}}$, например, у у-переменной №1 может быть меньше значимых «весов», чем у упеременной №4. В то время как вариабельность переменной №1 больше, чем вариабельность переменной №4. Переменная $\mathrm{z}_{\mathrm{j}}$ имеет смысл, если она обладает значимым «весом» $\mathrm{c}_{1 \mathrm{j}}$, присутствующим с любым значением коэффициента «изменчивости» $\mathrm{Z}_{\mathrm{kj}}$ при $\mathrm{Z}_{\mathrm{i} 1} \mathrm{c}_{1 \mathrm{j}}$. Первичен значимый «вес» $\mathrm{c}_{1 \mathrm{j}}$, вторична величина коэффициента «изменчивости» $\mathrm{z}_{\mathrm{kj}}$. Количество таких произведений с значимыми [4] «весами» при $\mathrm{z}$-переменных в линейной комбинации вида $\mathrm{y}_{\mathrm{ij}}=\mathrm{z}_{\mathrm{i} 1} \mathrm{c}_{1 \mathrm{j}}+\mathrm{z}_{\mathrm{i} 2} \quad \mathrm{c}_{2 \mathrm{j}}+\ldots+\mathrm{z}_{\mathrm{in}} \mathrm{c}_{\mathrm{nj}}$ может быть равно $\ell=1,2,3,4$.

В [4] найдены смыслы для комбинаций смыслов Z-переменных из матрицы $\mathrm{C}_{\mathrm{nn}}$ из ПМ ГК [2]. В ОС36 наоборот: надо задавать смыслы и имена у-переменным, z-переменным и назначить весомые «веса» в матрице $\mathrm{C}^{+}{ }_{n}$. Реализация при входном объекте $\mathrm{C}^{+}{ }_{n n}$ Обратной Моделей Главных Компонент дает нам основание проверять на адекватность реальной многомерной выборке нашу модельную выборку $\mathrm{Z}_{\mathrm{mn}}=\mathrm{U}_{\mathrm{mn}}\left[\mathrm{C}^{+}{ }_{\mathrm{nn}}\right]^{\mathrm{T}}$. Если извлеченные знания из матрицы псевдособственных векторов $\mathrm{C}^{+}{ }_{\mathrm{nn}}$ имеют приблизительно те же когнитивные смыслы и названия (имена) $\mathrm{z}$-переменных, соответствующих смыслу своей у-переменной, равной линейной комбинации $\mathrm{Z}$-переменных, то достигнута смысловая адекватность знаний.

Полученные в результате матрицы $\Lambda^{+}=\mathrm{I}_{\mathrm{nn}} \quad$ и $\mathrm{C}^{+}{ }_{\mathrm{nn}}$ используются для моделирования многомерных выборок $\mathrm{Z}_{\mathrm{mn}}, \mathrm{Y}_{\mathrm{mn}}$. Матрица $\mathrm{C}_{\mathrm{nn}}^{+}$ является объектом, из которого извлекаются цифровые знания [3-7]. Весомому значению компоненты $\mathrm{c}_{\mathrm{k} 1}$ псевдособственного вектора $\mathbf{c}_{1}=\left(\mathrm{c}_{11}, \mathrm{c}_{21} \ldots \mathrm{c}_{\mathrm{n} 1}\right)^{\mathrm{T}}$, по абсолютной величине удовлетворяющие критерию $\operatorname{abs}\left(\mathrm{c}_{\mathrm{k} 1}\right) \geq \mathrm{c}_{0}$, $\mathrm{k} \in\{1, \ldots, \mathrm{n}\}$, ставится в соответствие найденный смысл. Рассматриваются $\ell$ штук наборов пар $\left(\lambda_{\mathrm{j}}=1, \mathbf{c}_{\mathrm{j}}\right), \quad \mathrm{j}=1, \ldots, \quad \ell$. Для каждой пары $\left(\lambda_{j}=1, \mathbf{c}_{\mathbf{j}}\right)$, строится орграф когнитивных связей j-ой $\mathrm{y}$-переменной с z-переменными $\mathrm{z}_{\mathrm{k}}$, где номер $\mathrm{k} \in\{1, \ldots, \mathrm{n}\}$. Номер j у-переменной называется jым узлом орграфа. Эти орграфы образуют когнитивную карту, при этом обязательно приводится математическая модель, связывающая у-переменную с значениями коэффициентов изменчивостей $\mathrm{z}$-переменных $\mathrm{Z}_{\mathrm{k}}, \mathrm{k} \in\{1, \ldots, \mathrm{n}\}$, обязательно имеющих весомый «вес» из матрицы псевдособственных векторов $\mathrm{C}^{+}{ }_{n n}$. Примеры узлов орграфов приведены в работах [ 15].

Наши модельные $\mathrm{C}_{\mathrm{nn}}^{+}$-выборки $\mathrm{Z}^{(\mathrm{t})}{ }_{\mathrm{mn}}=\mathrm{U}^{(\mathrm{t})}{ }_{\mathrm{mn}}\left[\mathrm{C}^{+}{ }_{\mathrm{nn}}\right]^{\mathrm{T}}, \mathrm{t}=1, \ldots, \mathrm{k}_{\mathrm{t}}$, воспроизведенные не по известному спектру корреляционной матрицы, наравне с $\left(\Lambda=\mathrm{I}_{\mathrm{nn}}\right)-$ выборками, будут использованы при решении задач «извлечения цифровых знаний», исходя из своего "невиртуального" представления, из цифровых данных из разных предметных областей, средствами когнитивного моделирования. Данные должны быть организованы в виде таблицы объект-свойства.

\section{References:}

1. Hotelling, H. (1933). Analysis of a complex of statistical variables into principal components. J.Educ. Psych., v.24, 417,441,498-520.

2. Zhanatauov, S. U. (2013). Obratnaya model' glavnykh komponent. (p.201). Almaty: Kazstatin-form.

3. Chalmers, C. P. (1975). Generation of correlation matrices with a given eigenstructure. J. Stat. Comp. Simul., vol.4, 133-139.

4. Zhanatauov, S. U. (2017). The optimization problem with linearized equations f-parameters (f1,f2,f3,f4,f5,f6)-spectrum. International scientific journal Theoretical \&Applied Science, №11, vol.55, 251-267. www.t-science.org
5. Zhanatauov, S. U. (2017). Optimization problem of modeling missing elements of the spectrum of the correlation matrix. International scientific journal Theoretical \&Applied Science, №10, vol.54, 189-198. www.t-science.org

6. Zhanatauov, S. U. (2018). Inverse spectral problem. Int. Scientific Journal Theoretical \&Applied Science, №12(68), 101-112. www.tscience.org

7. Zhanatauov, S. U. (2016). Modeling eigenvectors with given the values of their indicated components. International Scientific Journal Theoretical \&Applied Science, №11, vol.43, 107-119. www.T-Science.org 


\begin{tabular}{|c|c|c|c|c|c|c|}
\hline \multirow{4}{*}{ Impact Factor: } & ISRA (India) & $=\mathbf{3 . 1 1 7}$ & SIS (USA) & $=0.912$ & ICV (Poland) & $=6.630$ \\
\hline & ISI (Dubai, UAE & $=0.829$ & РИНЦ (Russia & $=0.156$ & PIF (India) & $=1.940$ \\
\hline & GIF (Australia) & $=0.564$ & ESJI (KZ) & $=8.716$ & IBI (India) & $=4.260$ \\
\hline & JIF & $=1.500$ & SJIF (Morocco & $=5.667$ & OAJI (USA) & $=0.350$ \\
\hline
\end{tabular}

8. Zhanatauov, S. U. Inverse spectral problem with indicated values of components of the eigenvectors. Int. Scientific Journal Theoretical\&Applied Science, №11(67), 359370. www.t-science.org

9. Zhanatauov, S. U. (2018). Model of digitalization of the validity indicators and of the measurable indicators of the enterprise. Int.Scien.Jour. Theoretical \&Applied Science, № 9(65), 315-334. www.T-Science.org

10. Zhanatauov, S. U. (2018). Model of digitalization of indicators of individual consciousness. ISJ Theoretical \&Applied Science, №6(62), 101-110. www.t-science.org

11. Zhanatauov, S. U. (2014). The inverse problem, inverse model, invertible model. «Internat $\mathrm{C}$ onference " Science: lntegrating Theory and Practice" (February 24-25. 2014). (pp.447-449). Bozeman, Montana, USA/ ICET ( International enterf or Education\&Technology USA) Iternational Academic Research Conference on Business, Education, Nature and Technology».

12. Zhanatauov, S. U. (2013). Kognitivnaya karta i model' sotsial'no-ekonomicheskikh faktorov kar'ernoy uspeshnosti shkol'nikov munitsipal'nykh shkol SShA. Sibirskiy pedagogi cheskiy zhurnal, №6, 28-33.

13. Zhanatauov, S. U. (2015). Kognitivnaya karta $i$ kognitivnaya model' analiza glavnykh komponent (telekommuni-katsionnaya otrasl'). Natsional'naya assotsiatsiya uche-nykh (NAU).IX Mezhd. nauch.-prakt. konf: «Otechestvennaya nauka v epokhu izmeneniy: postulaty proshlogo i teorii novogo vremeni». (pp.55-58). Rossiya, Ekaterinburg,16-17 maya 2015.

14. Zhanatauov, S. U. (2014). Analiz budushchikh debitorskoy i kreditorskoy zadolzhennostey munitsi palitetov gorodov. Ekonomicheskiy analiz:teoriya i praktika. Moscow: №2(353), 5462. www.fin-izdat.ru/journal/analiz/

15. Zhanatauov, S. U. (2018). Model of digitalization of the validity indicators and of the measurable indicators of the enterprise. Int.Scien.Jour. Theoretical \&Applied Science, № 9(65), 315-334. www.T-Science.org

16. Axelrod, R. (1976). The Structure of Decision: Cognitive Maps of Political Elites. Princeton. Univ.Press. 